\title{
Investigations of sirtuin metabolism
}

\author{
Dissertation \\ zur Erlangung des mathematisch-naturwissenschaftlichen Doktorgrades \\ "Doctor rerum naturalium" \\ der Georg-August-Universität Göttingen \\ im Promotionsprogramm "Grundprogramm Biologie" \\ der Georg-August University School of Science (GAUSS) \\ vorgelegt von \\ Svenja Heitmüller \\ aus Hameln \\ Göttingen, 2014
}




\section{Betreuungsausschuss}

Jun.-Prof. Dr. Heinz Neumann, Angewandte Synthetische Biologie, Institut für Mikrobiologie und Genetik, Georg-August-Universität Göttingen

Prof. Dr. Kai Tittmann, Bioanalytik, Albrecht-von-Haller-Institut für Pflanzenwissenschaften, Georg-August-Universität Göttingen

\section{Mitglieder der Prüfungskommission}

Referent:

Jun.-Prof. Dr. Heinz Neumann, Angewandte Synthetische Biologie, Institut für Mikrobiologie und Genetik, Georg-August-Universität Göttingen

Korreferent:

Prof. Dr. Kai Tittmann, Bioanalytik, Albrecht-von-Haller-Institut für Pflanzenwissenschaften, Georg-August-Universität Göttingen

Weitere Mitglieder der Prüfungskommission:

Prof. Dr. Jörg Stülke, Allgemeine Mikrobiologie, Institut für Mikrobiologie und Genetik, Georg-August-Universität Göttingen

Prof. Dr. Ralf Ficner, Molekulare Strukturbiologie, Institut für Mikrobiologie und Genetik, Georg-August-Universität Göttingen

PD Dr. Michael Hoppert, Allgemeine Mikrobiologie, Institut für Mikrobiologie und Genetik, Georg-August-Universität Göttingen

Prof. Dr. Matthias Dobbelstein, Molekulare Onkologie, Göttinger Zentrum für Molekulare Biowissenschaften, Georg-August-Universität Göttingen

Tag der mündlichen Prüfung: 02. Juli 2014 




\section{Table of contents}

1 Introduction

1.1 Lysine acylations on histones ___ 1

1.2 Histone deacetylases __ 2

1.2.1 Sirtuins _ 3

$1.3 O$-acetyl-ADP-ribose formation and proposed functions in cell metabolism _ 4

1.4 Engineering of the $O$-acetyl-ADP-ribose synthase ___ 10

1.5 Objectives __ 11

2 Material and Methods _ 13

2.1 Material__ 13

2.1.1 Technical apparatures___ 13

2.1.2 Chromatography columns and resins ___ 14

2.1.3 Kit systems __ 14

2.1.4 Chemicals _ 15

2.1.5 Other material___ 15

2.1.6 Buffers and Solutions __ 16

2.1.7 SDS-PAGE gel composition (for six gels) ___ 18

2.1.8 Media and culture plates __ 19

2.1.9 Antibiotics _ 19

2.1.10 Antibodies__ 19

2.1.11 Cell strains __ 20

2.1.12 Enzymes and DNA-, protein size-standards __ 20

2.1.13 DNA Oligonucleotides___ 21

2.2 Methods _ 22

2.2.1 Molecular biological methods __ 22

2.2.1.1 Polymerase Chain Reaction (PCR) _ 22

2.2.1.2 Restriction digest __ 24

2.2.1.3 Ligation __ 24 
2.2.1.4 Agarose gel electrophoresis 24

2.2.1.5 DNA extraction from agarose gels _ـ 24

2.2.1.6 Plasmid DNA preparation __ 25

2.2.1.7 Determination of DNA concentration__ 25

2.2.1.8 Sequencing _ 25

2.2.1.9 RNA Isolation___ 26

2.2.1.10 cDNA transcription __ 27

2.2.2 Microbiological methods__ 27

2.2.2.1 Chemical competent cells ___ 27

2.2.2.2 Transformation of plasmid DNA into chemical competent cells ___ 27

2.2.2.3 Protein expression 28

2.2.2.4 Cell disruption _ 30

2.2.3 Protein biochemical methods __ 30

2.2.3.1 SDS gel electrophoresis (SDS-PAGE) _ 30

2.2.3.2 Immunoblot (Western Blot) __ 30

2.2.3.3 Protein purification __ 32

2.2.3.4 Size exclusion chromatography __ 36

2.2.4 Spectroscopic methods and assays__ 36

2.2.4.1 Enzymatic coupled sirtuin assay ___ 36

2.2.4.2 Resazurin-Assay___ 38

2.2.4.3 NAD ${ }^{+}$-Assay __ 39

2.2.4.4 Activity test of pncA-MBP _ 39

2.2.4.5 Lysine deacylation assay ___ 40

2.2.5 One-step synthesis of the metabolite O-acetyl-ADP-ribose ___ 41

3 Results 43

3.1 The $O$-acetyl-ADP-ribose synthase (OAADPRS)___ 43

3.1.1 Engineering and functional optimization of the $O$-acetyl-ADP-ribose synthase___ 43

3.1.2 Validation of the formation of $O$-acetyl-ADP-ribose 
3.1.3 Kinetic characterization of the optimized synthase 1.1 55

3.1.4 Effects of the synthase and the formation of the metabolite OAADPR in Escherichia coli 58

3.1.4.1 Gene expression analysis 65

3.1.4.2 Colorimetric ß-galactosidase assay 69

3.1.4.3 Transcription analysis via real-time PCR 72

3.1.5 Chemical synthesis of the metabolite $O$-acetyl-ADP-ribose 76

3.2 Removal of acyl modifications on histone H4K16 by sirtuins 81

4 Discussion 87

4.1 The functional optimized $O$-acetyl-ADP-ribose synthase 1.1 and its characterization 87

4.2 Analysis of the effects of synthase 1.1 expression and OAADPR formation 89

4.3 Removal of acyl modifications on histone H4K16 by sirtuin CobB 98

5 Summary 103

6 References 105

7 Supplementry___ 117

7.1 Table of figures 117

7.2 Abbreviations 125

7.3 Supplemetry data 128

7.3.1 Gene expression analysis 128

7.4 Acknowledgements 137

7.5 Curriculum vitae 139 



\section{Introduction}

\subsection{Lysine acylations on histones}

In eukaryotes, nucleosomes are the basic unit of DNA packing and form the first step of DNA compaction leading to chromosome formation. ${ }^{1}$ They consist of approximately 147 base pairs of DNA which is wound around a protein octamer. This octamer is composed of two molecules each of the four core histones $\mathrm{H} 2 \mathrm{~A}, \mathrm{H} 2 \mathrm{~B}, \mathrm{H} 3$ and $\mathrm{H} 4$. Single nucleosomes are connected by short DNA segments into nucleosomal arrays (Figure 1.1).

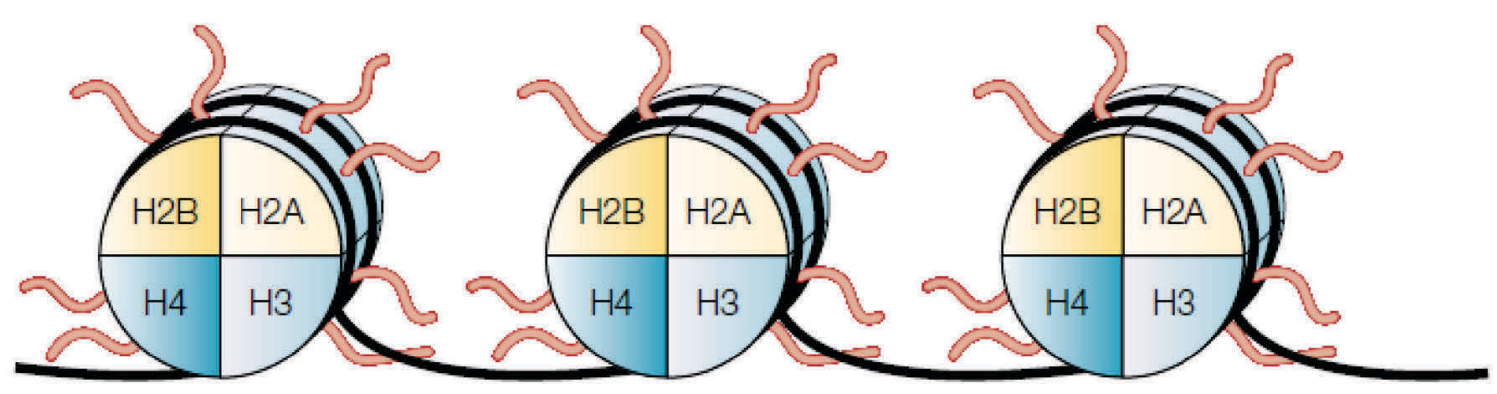

Figure 1.1: Schematic structure of a nucleosomal array. ${ }^{2}$ The DNA (black) is wrapped around the four core histones $\mathrm{H} 2 \mathrm{~A}, \mathrm{H} 2 \mathrm{~B}, \mathrm{H} 3$ and $\mathrm{H} 4$, which are present in two copies. The $\mathrm{N}$-terminal tails of the histones (rose) are oriented out of the octamer.

All four core histones have N-terminal tails, which are oriented out of the octamer and solvent exposed. On these $\mathrm{N}$-terminal tails most of the posttranslational modification sites of the core histones can be found. Posttranslational modifications (PTMs) are important for the modulation of chromatin structure and function. Over the last decades a vast number of PTMs on histones have been identified. One of the first modifications described was the acetylation of lysine residues in the N-terminal tails of histones $\mathrm{H} 3$ and $\mathrm{H} 4 .^{3-6}$ Acetyl modifications of lysines play an important role in chromatin regulation and correlate with active and open chromatin. ${ }^{7}$ They are also associated with DNA repair. ${ }^{8}$ Besides the well known PTMs on lysines like acetylation, methylation and ubiquitination, novel modifications were discovered over the last years. ${ }^{9}$ They include different 
acylations like butyrylation, proionylation, crotonylation, succinylation and malonylation. ${ }^{10-12}$ A closer look on their molecular structure exhibits similarities between some of them. The butyryl and propionyl modifications only differ in the length of their acyl-side chain from the acetyl modification and crotonyl groups only obtain an additional bond compared to buturyl groups. Considering these similarities the question arose if they are removed by the same enzymes as acetyl modifications on lysines and if the enzymes obtain a specificity to discriminate between them. Studies demonstrated that many histone deacetylases, including sirtuins, remove different types of acylations but only few detailed studies about the kinetic of these reactions have been performed. Sirtuins couple their activity to the formation of an small acylated molecule. Depending on the acylation of the substrate, different variants of this molecule are produced but it is unlcear if different acyl groups alter the properties of the molecule. In general, most effects of the different histone lysine acylations on cellular processes are still unclear.

\subsection{Histone deacetylases}

One family of enzymes that catalyzes the cleavage of PTMs are histone deacetylases (HDACs). They hydrolyze acetyl groups from the $\varepsilon-N$-acetyllysine amino acid in histones to tighten DNA binding and promote chromatin condensation. Other known targets also include non-histone proteins like DNA binding transcription factors, structural proteins and nuclear import factors. ${ }^{13}$ HDACs are divided in four different classes by function and DNA sequence similarity (Table 1.1). ${ }^{14-17}$ These classes are named after their members in budding yeast Saccharomyces cerevisiae. The class III HDACs occupy an unique position in the family of HDACs because of their sequence composition and reaction mechanism. 
Table 1.1: Overview of the four HDAC classes. ${ }^{14-16}$ Depicted members of class III HDACs are the most prominent representatives in their class.

\begin{tabular}{lll}
\hline class & & members \\
\hline I & Rpd3-like & HDAC 1-3 \\
& & HDAC 4, 5, 8 \\
II & Hda1-like & HDAC 7 \\
& & HDAC 9 \\
& & HDAC 6, 10 \\
& & mammalian (SIRT1 - 7) \\
III & Sirtuins & Sir2 of S. cerevisiae \\
& & HDAC 11 \\
\hline
\end{tabular}

\subsubsection{Sirtuins}

Class III HDACs are similar to their founding member yeast Sir2 (Silent mating type information regulator 2). For this reason they are known as sirtuins. In contrast to the first two classes, which share limited sequence similarities, sirtuins do not show any sequence resemblance to other HDACs in the family. ${ }^{18}$ Within their class the catalytic core sequence is conserved from bacteria to humans and they reside mainly in the nucleus, mitochondria and cytoplasm. ${ }^{19,20}$ Sirtuins also resemble a special class inside the HDAC family because their reaction mechanism differs from other classes. Class I and II HDACs catalyze the direct hydrolysis of acetyllysine by utilization of an active-site zinc motif and generate deacetylated lysine and acetate. ${ }^{20}$ Sirtuins on the other hand deacetylate lysine residues in a chemical reaction without participation of an active-site zinc motif and utilize $\mathrm{NAD}^{+}$as co-substrate releasing nicotinamide and $O$-acetyl-ADP-ribose, in addition to the deacetylated product. ${ }^{21}$ At first sight the consumption of $\mathrm{NAD}^{+}$for the hydrolysis of an acetyl group appears to be inefficient, since the energy release is in a comparable range to hydrolysis of ATP to $\mathrm{ADP}^{22,23}$ and a dissipation of precious cellular resources. ${ }^{24}$ This suggests that the reaction plays an important role for the cell. On the one hand, the requirement of $\mathrm{NAD}^{+}$ensures the coordination of the $\mathrm{NAD}^{+}: \mathrm{NADH}$ ratio and therefore the 
metabolic energy status with protein deacetylation. This prevents inappropriate or mistimed activity of the sirtuin enzyme and provides a level of control that is missing for class I and II HDACs. ${ }^{24}$ This specialised mechanism results in the unique formation of the metabolite $O$-acetyl-ADP-ribose (OAADPR).

\subsection{O-acetyl-ADP-ribose formation and proposed functions in cell metabolism}

Functional analysis connects this small molecule with metabolic flux, gene expression, cellular redox processes and aging, cell cycle control and apoptosis. The discovery of the metabolite in the year 2000 emanated from investigations of the enzymatic functions of yeast Sir2. Initially, Sir2 was reported to possess a protein ADP-ribosylation activity with the requirement of $\mathrm{NAD}^{+22,25}$ Further research, however, revealed its more robust activity as an $\mathrm{NAD}^{+}$-dependent histone deacetylase. ${ }^{26-28}$ The utilization of $\mathrm{NAD}^{+}$as a co-factor, which is unique to the sirtuin class, finally led to the discovery of OAADPR. Tanner et al. investigated the intrinsic catalytic mechanism of Sir2 and wanted to authenticate the reaction products. To their surprise, they did not find significant amounts of ADP-ribose (ADPR) and acetate, which were two of the predicted products, but an adduct between them. This adduct was identified by mass spectrometry as an acetyl-ADP-ribose, the metabolite OAADPR.

One of the first experiments performed after the discovery of this novel metabolite was a quantitative microinjection assay of OAADPR in starfish oocytes. It was shown that the metabolite, as well as purified sirtuin, could delay or even block maturation of oocytes and blastomeres. This supported the idea that the metabolite possesses biological activity as a novel signaling molecule or second messenger in a tightly controlled metabolism. ${ }^{29}$ Since then, several OAADPR-metabolizing enzymes have been reported. One of the best studied families are the NUDIX hydrolases (hydrolysis of a nucleoside diphosphate linked to another moiety $\underline{\mathrm{x}})^{30}$ They cleave the pyrophosphate bond of OAADPR, generating 2and 3-O-acetylribose-5-phosphat and adenosine monophosphate (AMP). Other known 
OAADPR-metabolizing enzymes are ARH3 (ADP-ribosylhydrolase $\underline{3}$ ) and macrodomain proteins. They hydrolyze the acetyl group of OAADPR and form free acetate and ADPR. $^{31,32}$

The unique involvment of $\mathrm{NAD}^{+}$and the formation of a novel metabolite in sirtuin catalyzed deacetylation reactions has led to closer investigations of the underlying catalytic mechanism. Several assumptions for different types of mechanisms have been reported. The proper identification of 2'-OAADPR was one of the key steps that led to the following proposed mechanism (Figure 1.2). ${ }^{33,34}$ The initial step of the deacetylation reaction involves a nucleophilic addition of the acetyl oxygen to the $\mathrm{C1}^{\prime}$ atom of the nicotinamide ribose to form a $\mathrm{C}^{\prime}$-O-alkylamidate intermediate. Both $\mathrm{S}_{\mathrm{N}} 1$ and $\mathrm{S}_{\mathrm{N}} 2$ mechanisms were proposed for this nucleophilic attack. A study using kinetic isotope analysis and computational models suggested a concerted, but highly asynchronous substitution mechanism. ${ }^{35}$ Following the alkylamidate formation, the 2 '-hydroxyl group of the $\mathrm{NAD}^{+}$ribose is activated by a conserved histidine in the $\mathrm{NAD}^{+}$binding pocket. The activated hydroxyl group attacks the $O$-alkylamidate carbon to form a $1^{\prime}, 2^{\prime}$-cyclic intermediate. ${ }^{36}$ Finally, a base-activated water molecule attacks the cyclic intermediate, releasing deacetylated lysine and 2'-OAADPR. Subsequently, at neutral $\mathrm{pH}, \mathrm{2}^{\prime}$-OAADPR undergoes a non-enzymatic transesterification yielding $2^{\prime}$ - and $3^{\prime}$-OAADPR in a 1:1 molar ratio. ${ }^{21,33}$ 


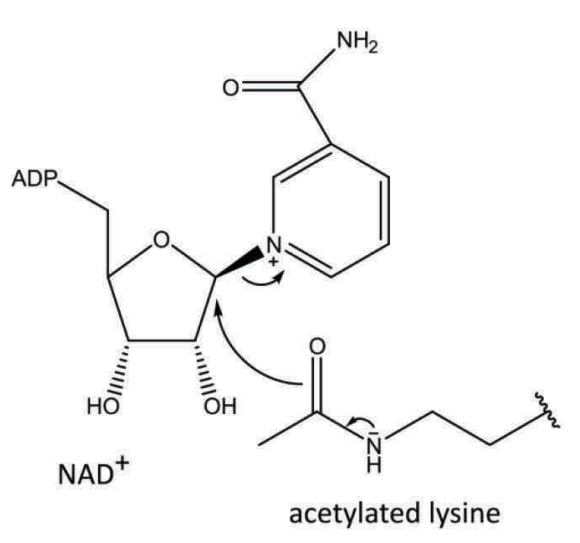

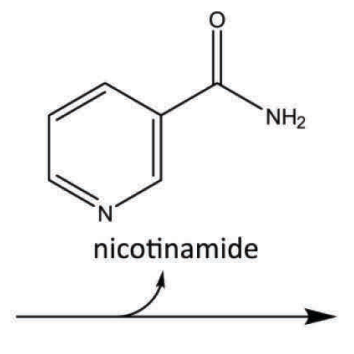

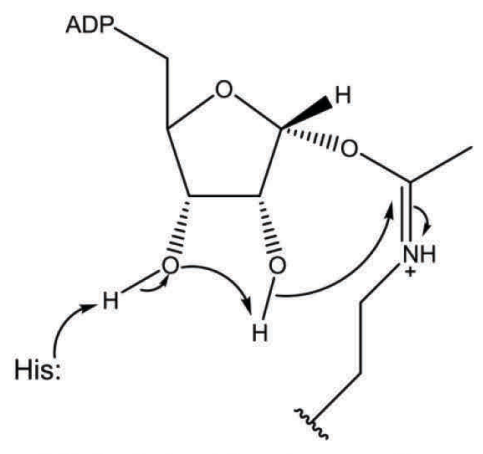

C1'-O-alkylamidate intermediate

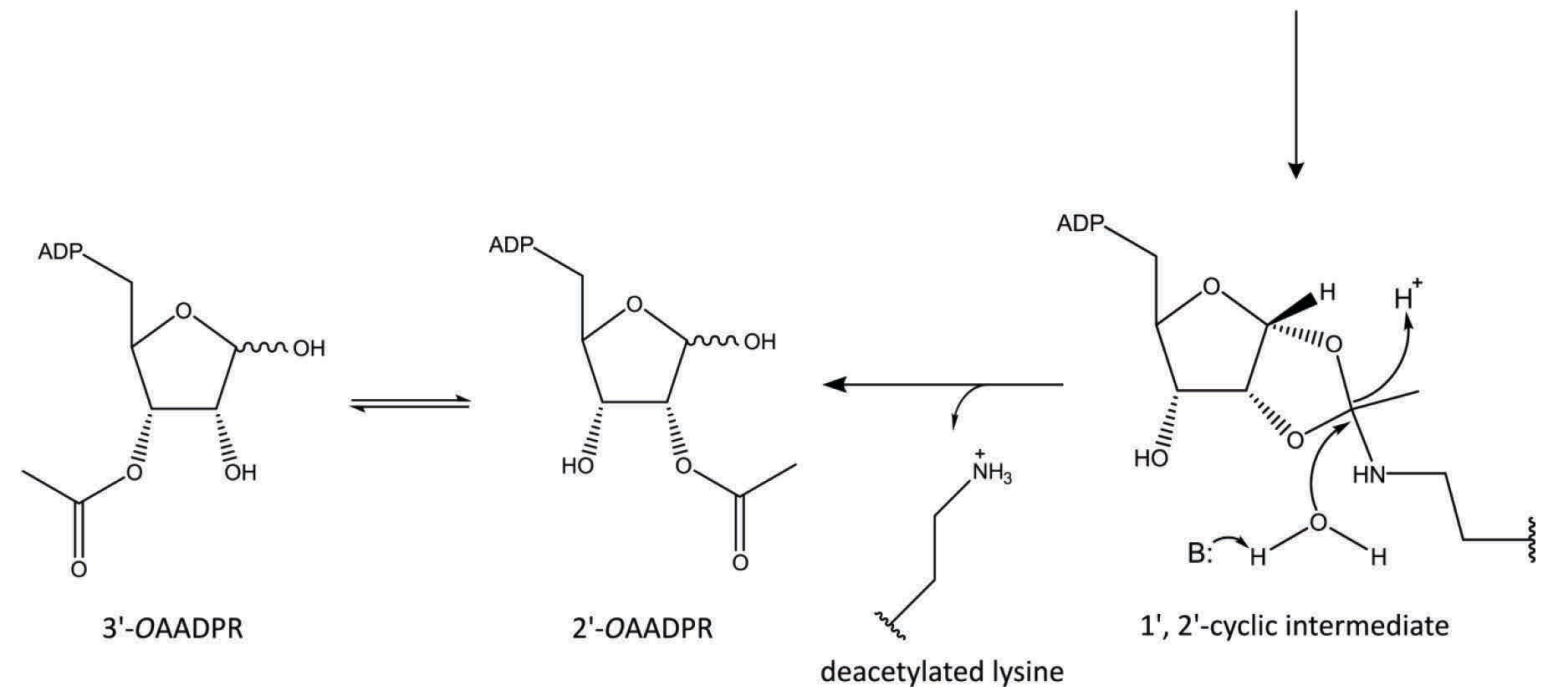

Figure 1.2: Proposed catalytic mechanism of OAADPR formation. ${ }^{\text {modifier after } 37}$ At first the oxygen of the acetyllysine attacks the $\mathrm{C}^{\prime}$ atom at the nicotinamide ribose and nicotinamide is released. The hydroxyl group at C2' gets activated by a conserved histidine, attacks the carbon atom of the $O$-alkylamidate and a $1^{\prime}, 2^{\prime}$-cyclic intermediate is formed. This intermediate is attacked by a base-activated water molecule which leads to the formation of 2'-OAADPR and the release of deacetylated lysine. A non-enzymatic transesterification yields a mixture of 2'- and 3'-OAADPR.

As mentioned above, OAADPR is thought to function as a signalling molecule or second messenger in many cellular processes, which includes gene silencing, ion channel activation and decreased reactive oxygen species (ROS) levels. ${ }^{37}$ Its regulatory function in gene silencing was unveiled in studies by Liou and McBryant in the years 2005 and 2006. ${ }^{38,39}$ They analyzed factors affecting the formation of the Silent Information 
Regulator (SIR) complex by utilizing an in vitro assembly approach. The SIR complex is a multi-protein nucleosomal binding complex and mediates silencing at telomeres and mating type loci in S. cerevisiae. It is composed of three sirtuins: Sir2, Sir3 and Sir4. ${ }^{40}$ Analyses by Liou and McBryant showed, that only the addition of acetyllysine substrate and $\mathrm{NAD}^{+}$increased the binding of Sir3 to the Sir2/Sir4 complex. This effect was suggested to be either due to $\mathrm{NAD}^{+}$hydrolysis or the formation of OAADPR. Addition of purified OAADPR resulting in the same increase of the binding effect supported its role in the structural arrangement of the SIR complex. Other studies reported OAADPR to be non-essential for Sir3 recruitment to the Sir2/Sir4 complex. ${ }^{41}$ Gasser et al. analyzed the effect in a nucleosomal context. They preincubated the SIR complex with OAADPR, which resulted in a subsequently increased affinity of the complex for the chromatin template. ${ }^{42}$ In summary, OAADPR presumably enhances the efficiency of Sir3 binding to Sir2/Sir4 and chromatin and therefore supports the formation of silent chromatin, although not being essential for these processes.

The ion channel activating function of OAADPR was investigated in 2006 by Grubisha et al. ${ }^{43}$ They discovered that the metabolite induces the channel gating process in TRPM2 (transient receptor potential malastatin-related channel 22). TRPM2 is a non-selective cation channel which is stimulated by oxidative and nitrative stress and supports susceptibility to cell death. ${ }^{44}$ For its activation, ADPR binds to the C-terminal cytoplasmic domain of the channel, which displays homology to a NUDIX hydrolase but without any enzymatic activity. ${ }^{45,46}$ OAADPR can bind to this domain as well. It modulates the gating in a similar way as ADPR but is possibly generated in higher amounts in response to increased sirtuin activity during metabolic adaption. ${ }^{43}$ A plausible explanation for putative modulation of TRPM2 by acetylated ADP-ribose might be a rapid degradation of OAADPR before binding to the C-terminal domain of the channel. ${ }^{30}$ Thus, ADPR would be accumulated and subsequently induce channel gating.

Tong et al. investigated the appearance of decreased ROS levels in the presence of OAAPR/ADPR. ${ }^{47}$ They analyzed S. cerevisiae $\triangle y s a 1$ cells in comparison to wild type strains. Ysa1 is a member of the NUDIX hydrolase family. $\Delta y s a 1$ cells, containing increased levels 
of OAAPR/ADPR, demonstrated a higher resistance to both endogenous and exogenous oxidative stress and possessed a general lower basal level of endogenous ROS. Two possible explanations were suggested for this mechanism (Figure 1.3). The first explanation is the inhibition of complex I of the electron transport chain. During transport from one complex to the next, the electrons can react with oxygen and form ROS. It was suggested that ADPR is able to inhibit the activity of complex I by preventing NADH from binding to the first complex and therefore electrons from passing on. ${ }^{48,49}$ Tong et al. could confirm ADPR as a physiological modulator of ROS originating from the electron transport chain. The second explanation for an increased oxidative stress resistance of $\Delta y s a 1$ cells is the promotion of pathways that suppress ROS damage and accumulation. ${ }^{47}$ It is well known that $\mathrm{H}_{2} \mathrm{O}_{2}$ - and $\mathrm{Cu}^{2+}$-stress lead to higher ROS levels and thus to cellular damage. To avoid this damage, yeast cells reroute the glucose mechanism from glycolysis to the NADPH-generating pentose phosphate pathway. ${ }^{50-53}$ The produced NADPH is required by reductases to reduce essential cellular antioxidants. ${ }^{54,55}$ OAADPR and ADPR are structurally similar to several coenzymes and substrates utilized by glycolytic enzymes and might modulate their activity. They were found to interact with the central glycolytic enzymes phosphoglycerate kinase (PGK) and glyceraldehyde-3-phosphate dehydrogenase (GAPDH), as well as with the glycolysis-related alcohol dehydrogenase (ADH). Consistent with the explanation of rerouting glucose to the pentose phosphate pathway by OAADPR/ADPR accumulation, NADPH levels in $\triangle y s a 1$ cells were increased by about $75 \%$. This led to a stronger resistance of the cells to toxic effects of ROS induced stress and also enabled the cells to maintain a lower basal level of endogenous ROS. ${ }^{47}$ 


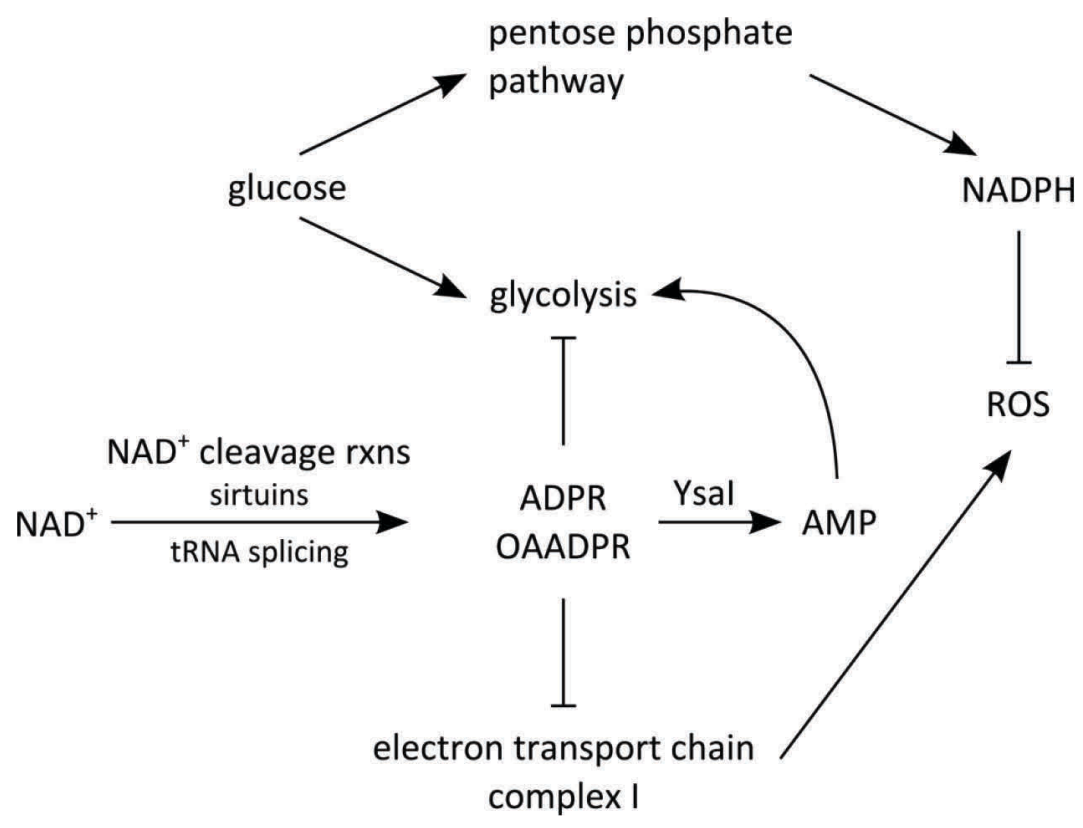

Figure 1.3: Proposed mechanisms of OAADPR/ADPR and Ysal function in metabolic pathways and cellular redox. $^{\text {modified after } 47}$ OAADPR/ADPR are generated by $\mathrm{NAD}^{+}$cleavage and hydrolyzed by Ysal to AMP which activates glycolysis. In $\triangle y s a 1$ cells OAADPR/ADPR levels are increased. They consequently inhibit glycolysis and promote NADPH production by rerouting glucose to the pentose phosphate pathway. This leads to a higher antioxidative stress response capability because essential cellular antioxidants can be reduced by the produced NADPH. Increased OAADPR/ADPR levels also inhibit complex I of the electron transport chain leading to a lower ROS level.

Despite several studies, the precise biological function of OAADPR and its molecular mechanism remains unclear. Also the full identification of interacting proteins is likely incomplete. Detailed analysis is generally hindered by the instability of OAADPR due to hydrolysis by several enzymes and the limited quantity available from purification of enzymatic reaction products. In vivo studies are generally hampered by the inability of the metabolite to permeate the plasma membrane. An approach to the problem of limited availability and instability was undertaken by Comstock et al. ${ }^{56}$ They developed a chemical synthesis of authentic OAADPR and two non-hydrolyzable analogues. The $O$ acetyl moiety of the analogues was replaced by an $\mathrm{N}$-acetyl group to stabilize them against spontaneous and enzyme-dependent hydrolysis. It also prevented the typically observed acyl migration with OAADPR. To obtain both 2'- and 3'-OAADPR analogues, the 
$\mathrm{N}$-acetyl groups were synthesized at position $2^{\prime}$ and $3^{\prime}$ of the ribose sugar. Validation of the ability of the two analogues to mimic OAADPR was demonstrated by binding studies with macroH2A1.1 histone protein. For resistance against the cleavage by NUDIX hydrolases, the P-O-P pyrophosphate moiety of the OAADPR analogue was replaced by a P-C-P moiety. ${ }^{57}$ These synthetic and non-hydrolyzable OAADPR analogues present valuable tools for studies of biological functions of the metabolite but are limited to in vitro studies.

\subsection{Engineering of the $O$-acetyl-ADP-ribose synthase}

An approach to overcome the problem of the membrane impermeability of the $O$-acetylADP-ribose synthase (OAADPR) is a major topic of this thesis. Since the metabolite cannot permeate the cell membrane, it needs to be produced in situ in the cell for investigations of its function in cell metabolism. Generally, the biosynthesis of sirtuins in the cell is uncomplicated but the control over their activity remains problematic. The co-substrate $\mathrm{NAD}^{+}$is universally available in the cell but sufficient primary substrate, the acetyllysine needs to be accessible to the sirtuin enzyme to produce beneficial amounts of the metabolite.

The solution for an in situ formation of the metabolite was to couple the primary substrate acetyllysine to the sirtuin enzyme and therefore enable a constant turnover without any external acetyllysine supplementation. For a functional reaction cycle the substrate lysine needs to be recharged with an acetyl group after every turnover. Therefore, an acetyltransferase had to be fused to the new enzyme as well. The aim of all this was to engineer an OAADPR synthase that combines deacetylase and acetyltransferase functionality linked by a peptide containing the substrate lysine for both moieties. This synthase should be able to produce the metabolite in situ and in defined levels. 


\subsection{Objectives}

The main objectives of this thesis were the engineering, functional optimization and characterization of a synthase that is capable of the in situ formation of the metabolite $O$-acetyl-ADP-ribose (OAADPR), and the realisation of experiments addressing the in vivo function of the metabolite. To this point, insights into the cellular functions and molecular mechanisms of this novel metabolite have been obtained from investigations of gene deletions affecting its metabolic rate and in vitro experiments. Since OAADPR is inable to permeate the plasma membrane and is susceptible to hydrolysis by several enzymes in its cellular environment, in vivo studies were hampered so far. A synthase that forms the metabolite within the cell and at defined levels would provide a solution to these problems. It would facilitate the evaluation of the obtained in vitro observations and analyses of the functions of OAADPR in vivo.

Furthermore, an objective was the analysis of the turnover rates of sirtuins on recently discovered acyl modifications on histone H4K16. The first method to create cleanly modified histone H4 protein, developed in Jun.-Prof. Heinz Neumanns group, allowed for the opportunity to investigate if sirtuins can discriminate between different acylations and to discover putative specificities. The results would provide a first step in the understanding of the effects of these acylations on nucleosome structure and function. 


\section{Material and Methods}

\subsection{Material}

\subsubsection{Technical apparatures}

AbiPrism 3100 DNA Sequencer

Agarose gel electrophoresis chamber

Äkta Prime

Autoclave HST 4-5-8

Bunsen burner Fuego basic

Centrifuge Allegra IR

Centrifuge Allegra J-20 XP

Centrifuge 5415 R

Centrifuge HEREAUS Pico 17

Centrifuge Speedvac 5301

Concentrators MWCO 10k, 30k

Electroblotter semi-dry

Electroblotter wet

FLUOstar Omega

GelDoc gel documentation device

Heating cabinet

Light table TFX-200LC

Magnetic stirrer MR Hei-Standard

NanoDrop ND-1000 Spectrophoto-

meter

Microfluidizer 110S

Optimax X-ray film processor

Orbital shaker Rotamax 120
Applied Biosystems, Darmstadt

GP-Kuststofftechnik, Kassel

GE Healthcare, München

Zirbus, Bad Grund

WLD-Tec, Göttingen

Beckman Coulter, Krefeld

Beckman Coulter, Krefeld

Eppendorf, Hamburg

Thermo Scientific, Langenselbold

Eppendorf, Hamburg

Sartorius, Göttingen

PeqLab, Erlangen

BioRad, München

BMG Labtech, Offenburg

BioRad, München

Mytron, Heiligenstadt

Vilber Lourmat, France

Heidolph, Schwabach

Thermo Scientific, Langenselbold

Microfluidics, USA

Protec, Oberstenfeld

Heidolph, Schwabach 
PCR cycler labcycler

pH-Meter PT-15

Photometer

Power Supply Consort EV231

Rotors JA-20, JLA-8.1000

Sonifier 250

Special accuracy balance

Spectrophotometer Ultrospec 2100

pro UV/Visible

Thermomixer comfort

Unitron shaking incubator

Vertical gel Electrophoresis chamber

H10 Mini

Vortexer VV3

\subsubsection{Chromatography columns and resins}

Amylose resin

Econo-Pac Chromatography columns

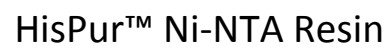

Superdex 75 26/70

Superdex 200 26/70

Nucleosil ${ }^{\circledR} 100-5 \mathrm{NH}_{2}$ HPLC column

PolyHYDROXYETHYL $A^{\text {TM }}$ 300-5 HILIC

column

\subsubsection{Kit systems}

EnzyChrom ${ }^{\text {TM }} \mathrm{NAD}^{+} / \mathrm{NADH}$ Assay Kit
SensoQuest, Göttingen

Sartorius, Göttingen

Eppendorf, Hamburg

Turnhout, Belgium

Beckman Coulter, Krefeld

Branson, USA

Sartorius, Göttingen

Biochrom, England

Eppendorf, Hamburg

Infors HT, Basel

GP-Kunststofftechnik, Kassel

VWR, Darmstadt

New England Biolabs, USA

BioRad, München

Thermo Scientific, USA

GE Healthcare, München

GE Healthcare, München

Macherey-Nagel, Düren

The Nest Group, USA

BioAssay Systems, USA 
QIAGEN Plasmid Mini Kit

QIAquick Gel Extraction Kit

QIAGEN PCR Purification Kit

qPCR Kit RealMasterMix SYBR ROX

Reverse Transcription Kit QuantiTect

RNA Extraction Kit
QIAGEN, Hilden

QIAGEN, Hilden

QIAGEN, Hilden

5PRIME, Germany

QIAGEN, Hilden

Macherey \& Nagel, Düren

\subsubsection{Chemicals}

All common chemicals were purchased either from AppliChem (Darmstadt), Merck (Darmstadt), Roth (Karlsruhe) or Sigma-Aldrich (Steinheim) with the quality pro analysis.

\subsubsection{Other material}

Amicon Ultra-15 Centrifugal Filter

Merck Millipore, Darmstadt

Units (NMWL $10 \mathrm{kDa}, 30 \mathrm{kDa}$ )

Coli Rollers Plating Beads

Novagen, USA

Half micro cuvettes

Sarstedt, Nümbrecht

Disposal bags

Sarstedt, Nümbrecht

Falcontubes (15 mL, $50 \mathrm{~mL})$

Sarstedt, Nümbrecht

Hamilton syringe $50 \mu \mathrm{L}$

Hamilton, USA

Hyperfilm ECL

GE Healthcare, UK

Immobilon-P Transfer Membrane

Merck Millipore,

Instant Blue

Biozol, Eching

Low binding E tips $10 \mu \mathrm{L}$

Sorenson BioScience, USA

Low binding microcentrifuge tubes

Sorenson BioScience, USA

$1.7 \mathrm{~mL}$

Micro tubes $1.5 \mathrm{~mL}, 2.0 \mathrm{~mL}$

Sarstedt, Nümbrecht

Nitrocellulose Transfer Membrane,

Whatman, Dassel 


\section{Protan}

PCR Softtubes $(0.2 \mathrm{~mL}$ )

Petri dishes $92 \times 16 \mathrm{~mm}$ with cams

Pipet Research $(2.5 \mu \mathrm{L})$

Pipets Research Plus (10, 100,

$1000 \mu \mathrm{L})$

Pipet tips

96-well Microplates

UV Cuvettes

Whatman 3MM Chr paper
Biozym, Austria

Sarstedt, Nümbrecht

Eppendorf, Hamburg

Eppendorf, Hamburg

Sarstedt, Nümbrecht

BD Falcon, USA

Eppendorf, Hamburg

Whatman plc, UK

\subsubsection{Buffers and Solutions}

\begin{tabular}{ll}
\hline Amylose resuspension buffer & $50 \mathrm{mM}$ Tris pH 7.5 \\
& $100 \mathrm{mM} \mathrm{NaCl}$ \\
& $1 \mathrm{mM} \mathrm{PMSF}$ \\
& $2 \mathrm{mM} \mathrm{DTT}$ \\
& Lysozyme \\
\hline Amylose wash buffer & $50 \mathrm{mM}$ Tris pH 7.5 \\
& $100 \mathrm{mM} \mathrm{NaCl}$ \\
& $2 \mathrm{mM}$ DTT \\
\hline Amylose elution buffer & Amylose wash buffer supplemented with \\
& $10 \mathrm{mM}$ Maltose \\
\hline Calcium chloride solution & $60 \mathrm{mM}$ CaCl \\
& $10 \mathrm{mM}$ Pipes \\
& $15 \%$ glycerol \\
\hline 10x DNA Loading dye for agarose gels & $20 \%$ Glycerol \\
& $0.25 \%$ Bromphenole blue \\
& $0.25 \%$ Xylene cyanol \\
\hline
\end{tabular}


Gel filtration buffer

\begin{tabular}{ll} 
& $50 \mathrm{mM} \mathrm{NaCl}$ \\
& $2 \mathrm{mM} \mathrm{Mg}(\mathrm{OAc})_{2}$ \\
& $5 \mathrm{mM} \mathrm{DTT}$ \\
\hline 1x Laemmli buffer & $25 \mathrm{mM}$ Tris base \\
& $192 \mathrm{mM}$ Glycine
\end{tabular}

$3.5 \mathrm{mM}$ SDS

4x Loading buffer for SDS gels

$50 \mathrm{mM}$ Tris $\mathrm{HCl}$ pH 6.8

100 mM DTT

$2 \%$ SDS solution

$0.1 \%$ Bromphenol blue

10\% Glycerol

Ni-NTA wash buffer $10 \mathrm{mM}$ Tris $\mathrm{HCl}$ pH 7.5

$200 \mathrm{mM} \mathrm{NaCl}$

$20 \mathrm{mM}$ Imidazole

$1 \mathrm{mM}$ DTT

1x PIC

Ni-NTA elution buffer NiNTA wash buffer supplemented with $220 \mathrm{mM}$ Imidazole

1x Phosphate-buffered saline (PBS) $137 \mathrm{mM} \mathrm{NaCl}$

$2.7 \mathrm{mM} \mathrm{KCl}$

$10 \mathrm{mM} \mathrm{Na}_{2} \mathrm{HPO}_{4}$

$1.76 \mathrm{mM} \mathrm{KH}_{2} \mathrm{PO}_{4}$ $\mathrm{pH} 7.4$

Ponceau S staining solution

$0.5 \%(w / v)$ Ponceau S pigment

$5 \%$ Trichloroacetic acid

1000x Protease Inhibitor Cocktail $75 \mathrm{mM}$ Pefablock $150 \mu \mathrm{M}$ Leupeptin hemisulfate salt $37.5 \mathrm{mM} \quad O$-Phenantrolin hydrochloridemonohydrate 


\begin{tabular}{ll}
\hline & $500 \mu M$ Pepstatin A \\
\hline 1x Running Buffer for SDS-PAGE & $25 \mathrm{mM}$ Tris base \\
& $192 \mathrm{mM}$ Glycine \\
& $3.5 \mathrm{mM}$ SDS \\
\hline 1x Stripping buffer for Western Blots & $200 \mathrm{mM}$ Glycine \\
& $3.5 \mathrm{mM}$ SDS \\
& $10 \%$ (v/v) Tween 20 \\
\hline 1x TBE buffer & $89 \mathrm{mM}$ Tris base \\
& $89 \mathrm{mM}$ Boric acid \\
& $2.5 \mathrm{mM}$ EDTA \\
\hline 1x Tris-buffered saline (TBS) & $50 \mathrm{mM}$ Tris HCl \\
& $150 \mathrm{mM}$ NaCl \\
\hline 1x Transfer Buffer (Western Blot) & $25 \mathrm{mM}$ Tris base \\
& $192 \mathrm{mM}$ Glycine \\
\hline $3.5 \mathrm{mM}$ SDS \\
$20 \%$ Methanol \\
\hline
\end{tabular}

\subsubsection{SDS-PAGE gel composition (for six gels)}

\begin{tabular}{llll}
\hline & Stacking gel & Separation gel & Separation gel \\
& $\mathbf{4} \%$ & $\mathbf{1 2 . 5} \%$ & $\mathbf{1 5}$ \\
\hline $\mathrm{H}_{2} \mathrm{O}$ & $12 \mathrm{~mL}$ & $21.8 \mathrm{~mL}$ & $16 \mathrm{~mL}$ \\
Tris-HCl & $5.0 \mathrm{~mL}$ & $17.6 \mathrm{~mL}$ & $17.6 \mathrm{~mL}$ \\
& $(0.625 \mathrm{M}, \mathrm{pH} 6.8)$ & $(1.5 \mathrm{M} . \mathrm{pH} 8.8)$ & $(1.5 \mathrm{M}, \mathrm{pH} 8.8)$ \\
$10 \%$ SDS & $200 \mu \mathrm{L}$ & $750 \mu \mathrm{L}$ & $750 \mu \mathrm{L}$ \\
Acrylamide/Bisacrylamide & $2.6 \mathrm{~mL}$ & $24.5 \mathrm{~mL}$ & $35 \mathrm{~mL}$ \\
$(37.5$ :1) & & & \\
$10 \%$ APS & $100 \mu \mathrm{L}$ & $750 \mu \mathrm{L}$ & $750 \mu \mathrm{L}$ \\
TEMED & $30 \mu \mathrm{L}$ & $60 \mu \mathrm{L}$ & $60 \mu \mathrm{L}$ \\
\hline
\end{tabular}




\subsubsection{Media and culture plates}

$1 \mathrm{~L}$ lysogenic broth (LB) medium contains:

\begin{tabular}{ll} 
Tryptone/Peptone & $10 \mathrm{~g}$ \\
Yeast Extract & $5 \mathrm{~g}$ \\
$\mathrm{NaCl}$ & $5 \mathrm{~g}$ \\
$\mathrm{Agar}$ & $1.5 \%$ for culture plates only \\
\hline
\end{tabular}

\subsubsection{Antibiotics}

\begin{tabular}{lll}
\hline Ampicillin & $50 \mu \mathrm{g} / \mathrm{mL}$ & AppliChem, Darmstadt \\
Kanamycin & $50 \mu \mathrm{g} / \mathrm{mL}$ & AppliChem, Darmstadt \\
Spectinomycin & $75 \mu \mathrm{gL}$ & Sigma, Steinheim \\
\hline
\end{tabular}

\subsubsection{Antibodies}

Anti-Histone $\mathrm{H} 4$ polyclonal antibody (rabbit)

Anti-Histone H4K16ac polyclonal antibody (rabbit)

Anti-crotonyllysine monoclonal antibody (clone 4D5) (mouse)

Anti-His monoclonal antibody (mouse)

Anti-Acetyllysine antibody (rabbit)

Anti-Mouse IgG (whole molecule)-Peroxidase antibody

Anti-Rabbit IgG (whole molecule)-Peroxidase antibody abcam, UK

Active Motif, USA

PTM Biolabs, USA

GE Healthcare, UK abcam, UK

Sigma Aldrich, USA

Sigma Aldrich, USA 


\subsubsection{Cell strains}
E. coli $\mathrm{BL} 21(\mathrm{DE} 3)$
E. coli $\mathrm{DH} 10 \mathrm{~B}$
E. coli C321. $\Delta$ A.exp

\subsubsection{Enzymes and DNA-, protein size-standards}

Acetyl coenzyme A sodium salt

Calf Intestinal Alkaline Phosphatase (CIP)

Lysozyme

L-Glutamic Dehydrogenase solution from Sigma-Aldrich, Steinheim bovine liver

Pfu Turbo DNA Polymerase

Phusion High-Fidelity DNA Polymerase

Proteinase K (recombinant), PCR grade

BamHI restriction enzyme

Xhol restriction enzyme

GeneRuler 100 bp Plus DNA Ladder

GeneRuler DNA Ladder Mix

QuickLoad 1 kb DNA Ladder

PageRuler Prestained Protein Ladder

T4 DNA ligase

Unstained Protein Molecular Weight Thermo Scientific, USA

Sigma-Aldrich, Steinheim

Thermo Scientific, USA

Agilent, USA

Thermo Scientific, USA

Thermo Scientific, USA

Thermo Scientific, USA

Thermo Scientific, USA

Thermo Scientific, USA

Thermo Scientific, USA

New England Biolabs, USA

Thermo Scientific, USA

Thermo Scientific, USA

Marker 


\subsubsection{DNA Oligonucleotides}

The DNA oligonucleotides (primers) were purchased from Sigma-Aldrich in desalted purity.

\begin{tabular}{|c|c|}
\hline Primer name & Sequence $5^{\prime} \rightarrow 3^{\prime}$ \\
\hline \multirow[t]{2}{*}{ Synthase 1.1} & CAGGAAGTCCGGAGTCAAGGGCTTGGGAAAGGGTG \\
\hline & CCTTGACTCCGGACTTCCTGGCTGCCTTGGTGGCC \\
\hline Synthase 1.1 & GGAGTCAAGGGCTTGGGCGGGGGTGGCGCCAAATC \\
\hline $\mathrm{K} \rightarrow \mathrm{G}$ mutant & GATTTGGCGCCACCCCCGCCCAAGCCCTTGACTCC \\
\hline \multirow[t]{2}{*}{ Synthase 1.2} & GGCCACCAAGGGAGTCAAGGGCTTGGGAAAGGGTG \\
\hline & CTTGACTCCCTTGGTGGCCAGCTGCTTGCGGGGAG \\
\hline \multirow[t]{2}{*}{ UhpT-lacZ fusion protein } & ATCAGTCGACGCGCGAAGCGTGATGCATCTCACC \\
\hline & TGATTCTAGAATACCTGCGTGAGGCATGCATTG \\
\hline \multirow[t]{2}{*}{ CobB } & CCAGGATCCAAAACCAAGAGTACTCGTACTGAC \\
\hline & GATTAAAAGCGGGAAGCATTGCCTAACTCGAGTCT \\
\hline \multirow[t]{2}{*}{ Sirt1 } & CCAAGGATCCTGTGAAAGTGATGAGGAGGATAGAG \\
\hline & CAGACTCGAGTTAGACGTCATCTTCAGAGTCTG \\
\hline \multicolumn{2}{|l|}{ Primers for real-time PCR } \\
\hline \multirow[t]{2}{*}{ uhpt } & TTCAGGGCTTTACGCTGTTTGA \\
\hline & ATTGTTGATTGGTGTGGCTGCT \\
\hline \multirow[t]{2}{*}{ nadA } & GGGCAGATTGGGTGGTAACTTC \\
\hline & AGTATGGCAGCATCCGGGTATT \\
\hline \multirow[t]{2}{*}{$\operatorname{nadB}$} & ATGTGGGATTACGTTGGCATTG \\
\hline & GAACAATCAACTCGGCAACCTG \\
\hline \multirow[t]{2}{*}{ pnuC } & TCTCTCATGGATCGAAGCGGTA \\
\hline & CCACGCATACCAGCCGTAAATA \\
\hline \multirow[t]{2}{*}{ fruB } & TCTGCTTTTCGCCCATCAGTAA \\
\hline & ACCGGCGTTCAGGTATTTCAGT \\
\hline \multirow[t]{2}{*}{ gapA } & GCCAGAACTGAATGGCAAACTG \\
\hline & TATCTACCGATTTCAACGGCGA \\
\hline
\end{tabular}




\subsection{Methods}

\subsubsection{Molecular biological methods}

\subsubsection{Polymerase Chain Reaction (PCR)}

DNA was selectively amplified from plasmids by polymerase chain reaction. ${ }^{58}$ The general setup for a PCR mixture $(50 \mu \mathrm{L})$ including Pfu Turbo DNA Polymerase (2.1.12) and the associated PCR cycling protocol is described below.

\begin{tabular}{lc} 
PCR mixture $(50 \mu \mathrm{L})$ & \\
\hline 10x cloned Pfu reaction buffer & $5.0 \mu \mathrm{L}$ \\
dNTPs (25 mM each dNTP) & $0.4 \mu \mathrm{L}$ \\
DNA template $(30 \mathrm{ng} / \mu \mathrm{L})$ & $1.0 \mu \mathrm{L}$ \\
Pfu Turbo DNA Polymerase $(2.5 \mathrm{U} / \mu \mathrm{L})$ & $1.0 \mu \mathrm{L}$ \\
Primer $1(25 \mu \mathrm{M})$ & $1.0 \mu \mathrm{L}$ \\
Primer $2(25 \mu \mathrm{M})$ & $1.0 \mu \mathrm{L}$ \\
Distilled water & $40.6 \mu \mathrm{L}$
\end{tabular}

\begin{tabular}{lrr}
\hline Cycle step & Temperature & Time \\
\hline Initial denaturation & $95{ }^{\circ} \mathrm{C}$ & $2 \mathrm{~min}$ \\
Denaturation & $95{ }^{\circ} \mathrm{C}$ & $30 \mathrm{~s}$ \\
Annealing & $\mathrm{Tm}-5^{\circ} \mathrm{C}$ & $30 \mathrm{~s}$ \\
Extension & $72{ }^{\circ} \mathrm{C}$ & $1 \mathrm{~min} / \mathrm{kb}$ \\
Final Extension & $72{ }^{\circ} \mathrm{C}$ & $10 \mathrm{~min}$ \\
& $4{ }^{\circ} \mathrm{C}$ & $\infty$
\end{tabular}


In the following a general setup for a PCR mixture $(50 \mu \mathrm{L})$ using Phusion DNA Polymerase and the associated PCR cycling protocol are described:

\begin{tabular}{lc}
\hline PCR mixture $(50 \mu \mathrm{L})$ & \\
\hline $5 x$ Phusion High Fidelity buffer & $10.0 \mu \mathrm{L}$ \\
dNTPs (10 mM each dNTP) & $1.0 \mu \mathrm{L}$ \\
DNA template $(10 \mathrm{ng} / \mu \mathrm{L})$ & $1.0 \mu \mathrm{L}$ \\
Phusion DNA Polymerase $(1 \mathrm{U} / \mu \mathrm{L})$ & $1.0 \mu \mathrm{L}$ \\
Primer $1(25 \mu \mathrm{M})$ & $1.0 \mu \mathrm{L}$ \\
Primer $2(25 \mu \mathrm{M})$ & $1.0 \mu \mathrm{L}$ \\
& \\
Distilled water & $35.0 \mu \mathrm{L}$
\end{tabular}

\begin{tabular}{lrr}
\hline Cycle step & Temperature & Time \\
\hline Initial denaturation & $98{ }^{\circ} \mathrm{C}$ & $30 \mathrm{~s}$ \\
Denaturation & $98^{\circ} \mathrm{C}$ & $5-10 \mathrm{~s}$ \\
Annealing & $\mathrm{Tm}+3{ }^{\circ} \mathrm{C}$ & $10-30 \mathrm{~s}$ \\
Extension & $72{ }^{\circ} \mathrm{C}$ & $15-30 \mathrm{~s} / \mathrm{kb}$ \\
Final Extension & $72{ }^{\circ} \mathrm{C}$ & $5-10 \mathrm{~min}$ \\
& $4{ }^{\circ} \mathrm{C}$ & $\infty$ \\
\hline
\end{tabular}

For purification of PCR products the QIAGEN PCR Purification Kit (QIAGEN, Hilden) was used following instructions of the manufacturer. 


\subsubsection{Restriction digest}

Restriction digest of DNA was performed following instructions of the restriction endonucleases manufacturer (Fermentas, now Thermo Scientific, USA). The digest was proceeded for three hours at $37^{\circ} \mathrm{C}$. Half an hour before end CIP (2.1.12) was added to remove 5'-phosphate groups from the template DNA to avoid self-ligation. In the final step the enzymes were deactivated by heating to $65^{\circ} \mathrm{C}$ for $15 \mathrm{~min}$.

\subsubsection{Ligation}

The reaction mixture for ligation of DNA fragments contains vector and insert DNA in a ratio of 1:3 together with $1 \cup$ T4 DNA ligase (2.1.12) and $2 \mu L$ T4 DNA ligase buffer. For a total volume of $20 \mu \mathrm{L}$ distilled water was added. The mixture was incubated at $37^{\circ} \mathrm{C}$ for $1.5 \mathrm{~h}$ and directly transformed into $E$. coli cells.

\subsubsection{Agarose gel electrophoresis}

For separation of DNA fragments gels with $1 \%(\mathrm{w} / \mathrm{v})$ agarose in $0.5 \mathrm{x}$ TBE buffer (2.1.6 Jwere used. After the agarose was dissolved in buffer by heating in the microwave, GelRed (1:20000) was added to the mixture for visualization of the DNA under UV light. DNA samples were mixed with 10x DNA loading dye and electrophoretically separated. Visualization of DNA bands was performed at a wavelength of $254 \mathrm{~nm}$ or $365 \mathrm{~nm}$ if DNA extraction was conducted afterwards.

\subsubsection{DNA extraction from agarose gels}

After a preparative restriction digest and electrophoretical separation of the DNA fragments in an agarose gel, bands of desired size were cut out under UV light ( $365 \mathrm{~nm}$ ). DNA was extracted from the gel pieces using the QIAquick Gel Extraction Kit (QIAGEN, Hilden) following the manufacturer's protocol. The purified DNA was eluted in $50 \mu \mathrm{L}$ 
$\mathrm{ddH}_{2} \mathrm{O}$ and the concentration measured with a photometer. The DNA was stored at $20^{\circ} \mathrm{C}$.

\subsubsection{Plasmid DNA preparation}

One single colony from a culture plate containing $\mathrm{DH} 10 \mathrm{~B}$ cells with the desired plasmid was transferred into a culture tube containing LB medium supplemented with antibiotic. The culture was incubated at $37{ }^{\circ} \mathrm{C}$ over night shaking. Cells were harvested by centrifugation (13300 rpm, $5 \mathrm{~min}$ ) at room temperature (RT). Plasmid DNA isolation and purification were performed using the QIAGEN Plasmid Mini Kit (QIAGEN, Hilden) according to manufacturer's protocol.

\subsubsection{Determination of DNA concentration}

The concentration of DNA in aqueous solutions was determined by measuring the absorption at $260 \mathrm{~nm}$ using a photometer (Eppendorf, Hamburg). The quotient of $260 \mathrm{~nm}$ to $280 \mathrm{~nm}$ displayed the purity of the DNA. A value between 1.8 and 2.0 indicated reasonably pure DNA, a lower value a contamination with proteins.

\subsubsection{Sequencing}

The principle of the Sanger dideoxy chain termination reaction was used for sequencing. Dideoxynucleotide triphosphate containing different fluorophors was incorporated into the DNA at random positions during PCR. This stopped the chain elongation due to a lacking 3 '-OH group to produce fragments which could be separated and analyzed. The PCR contained Seq-Mix BigDye Terminator v1.1 (Applied Biosystems, Darmstadt). The setup of the reaction mixture and the sequencing PCR are listed in Tables 2.1 and 2.2. 
Table 2.1 Setup of reaction mixture for PCR before sequencing.

\begin{tabular}{lr}
\hline Template DNA & $200-400 \mathrm{ng}$ \\
Primer & $8 \mathrm{pmol}$ \\
Seq-Mix & $1 \mu \mathrm{L}$ \\
Seq-Buffer & $1 \mu \mathrm{L}$ \\
$\mathrm{ddH}_{2} \mathrm{O}$ & Add to $10 \mu \mathrm{L}$ \\
\hline
\end{tabular}

Table 2.2: PCR program in preparation for sequencing.

$\left.\begin{array}{lcc}\hline \text { Cycle step } & \text { Temperature } & \text { Time } \\ \hline \text { Denaturation } & 96{ }^{\circ} \mathrm{C} & 10 \mathrm{~s} \\ \text { Annealing } & 55^{\circ} \mathrm{C} & 15 \mathrm{~s} \\ \text { Elongation } & 60{ }^{\circ} \mathrm{C} & 4 \mathrm{~min}\end{array}\right\} 25$ cycles

After the PCR $1 \mu \mathrm{L}$ EDTA (125 mM), $1 \mu \mathrm{L}$ sodium acetate (3 M) and $50 \mu \mathrm{L}$ ethanol (96\%) were added to the mixture. All components were mixed gently, incubated for 5 min and then centrifuged for $5 \mathrm{~min}$ at RT (13300 rpm). The supernatant was discarded, the pellet washed with $70 \mu \mathrm{L}$ ethanol (70\%) and centrifuged again. After removal of the supernatant the pellet was air dried and finally dissolved in $15 \%$ formamide (99.5\%). The containing DNA fragments were analyzed in a capillary sequencing instrument.

\subsubsection{RNA Isolation}

From cell culture $1 \times 10^{7}$ cells were harvested, mixed with $1 \mathrm{~mL}$ TRI reagent and incubated for $5 \mathrm{~min}$ at RT. $200 \mu \mathrm{L}$ chloroform were added and the mixture intensly shaken for $15 \mathrm{~s}$. After an incubation time of 2-15 min the sample was centrifuged $\left(4^{\circ} \mathrm{C}, 15 \mathrm{~min}\right.$, $13300 \mathrm{rpm}$ ). The liquid phase was transferred into a new tube, $500 \mu \mathrm{L}$ isopropanol added and incubated for $5-10 \mathrm{~min}$ at RT. Centrifugation $\left(4-25^{\circ} \mathrm{C}, 8 \mathrm{~min}, 13300 \mathrm{rpm}\right)$ resulted in the formation of a gel-like or white pellet. The supernatant was discarded. $1.2 \mathrm{~mL}$ ethanol $(75 \%)$ were added to the pellet and vortexed. After centrifugation $\left(4-25^{\circ} \mathrm{C}\right.$, 
$5 \mathrm{~min}, 8000 \mathrm{rpm}$ ) the pellet was air dried for 3 to $5 \mathrm{~min}$ and resuspended with a pipette in sterile water. The suspension was incubated for $10-15$ min at $55-60^{\circ} \mathrm{C}$. RNA concentration was measured in a photometer. A small fraction was run in a denaturing agarose gel for size control.

\subsubsection{0 cDNA transcription}

RNA was isolated as described above and transcribed into cDNA using the Reverse Transcription Kit QuantiTect (QIAGEN, Hilden). The transcription was performed following manufacturer's protocol.

\subsubsection{Microbiological methods}

\subsubsection{Chemical competent cells}

For the production of chemical competent cells $250 \mathrm{~mL}$ prewarmed LB medium was inoculated with cells from a preculture and incubated at $37^{\circ} \mathrm{C}$. At an $\mathrm{OD}_{600} 0.6-0.8$ the cells were harvested by centrifugation $\left(4^{\circ} \mathrm{C}, 15 \mathrm{~min}, 4800 \mathrm{rpm}\right)$. The cell pellet was washed with $10 \mathrm{~mL}$ cold calcium chloride solution (2.1.6) and centrifuged again. The washing step was repeated with an incubation time of $1 \mathrm{~h}$ on ice before centrifugation. Most of the supernatant was discarded and the cells resuspended in $2 \mathrm{~mL}$ remaining supernatant. Aliquots of $200 \mu \mathrm{L}$ were flash frozen in liquid nitrogen and stored at $-80^{\circ} \mathrm{C}$.

\subsubsection{Transformation of plasmid DNA into chemical competent cells}

Plasmid DNA was transformed into chemical competent cells via the heat shock method. Therefore $50 \mu \mathrm{L}$ cells were mixed with $5 \mu \mathrm{L}$ plasmid DNA and incubated for $10 \mathrm{~min}$ on ice. The 2 min heat shock was performed in the Thermomixer comfort (Eppendorf, Hamburg) at $42^{\circ} \mathrm{C}$. Cells were returned on ice, $1 \mathrm{~mL}$ LB medium without antibiotic added and 
incubated at $37^{\circ} \mathrm{C}$ for $1 \mathrm{~h}$ shaking. After recovery the cells were either plated on culture plates containing antibiotic or used for inoculation of a liquid preculture.

\subsubsection{Protein expression}

O-acetyl-ADP-ribose synthase

The different $O$-acetyl-ADP-ribose synthases in a pCDF Duet-1 vector were expressed in E. coli $\mathrm{BL} 21(\mathrm{DE} 3)$ cells. A colony was picked from a culture plate to inoculate $100 \mathrm{~mL} \mathrm{LB}$ medium supplemented with spectinomycin. This preculture was incubated at $37^{\circ} \mathrm{C}$ over night shaking. Next day the main culture, $500 \mathrm{~mL}$ LB medium with spectinomycin in a $2 \mathrm{~L}$ Erlenmeyer flask, was inoculated to $\mathrm{OD}_{600} 0.2$ and incubated at $37^{\circ} \mathrm{C}$ shaking. At $\mathrm{OD}_{600} 1.0$ protein expression was induced by addition of $0.5 \mathrm{mM}$ IPTG. Cells were harvested $4.5 \mathrm{~h}$ after induction, washed with cold PBS and stored at $-20^{\circ} \mathrm{C}$.

Expression of the synthases on a pBAD plasmid was also performed in E. coli BL21(DE3) cells. The preculture was prepared as described above with the antibiotic ampicillin. The main culture was inoculated at $\mathrm{OD}_{600} 0.1$ and incubated at $37^{\circ} \mathrm{C}$ shaking. At $\mathrm{OD}_{600} 0.3$ protein expression was induced by addition of $200-1000 \mathrm{ppm}$ arabinose. Cell samples were taken at defined time points depending on the assay they were used for.

CobB

Expression of CobB from the pCDF-CobB plasmid was performed as described above for OAADPRS on pCDF Duet-1 plasmid.

\section{Sirt1 constructs}

The three Sirt1 proteins with different length of $\mathrm{N}$ - and C-terminal regions were expressed in E. coli BL21(DE3) cells from a pCDF Duet-1 plasmid. Plasmid DNA was 
transformed into chemical competent cells via heat shock and used to inoculate $100 \mathrm{~mL}$ LB medium supplemented with spectinomycin. This preculture was incubated at $37^{\circ} \mathrm{C}$ over night shaking. The main culture contained $250 \mathrm{~mL}$ LB medium supplemented with spectinomycin and was inoculated to $\mathrm{OD}_{600} 0.2$. It was incubated at $37^{\circ} \mathrm{C}$ shaking up to $\mathrm{OD}_{600}$ 1.2. $250 \mathrm{~mL}$ cold $\mathrm{LB}$ medium with spectinomycin was added and the cell cultures were shifted to $18{ }^{\circ} \mathrm{C}$ before induction with $0.5 \mathrm{mM}$ IPTG. Cells were harvested the next day, washed with cold PBS and stored for short term at $-20^{\circ} \mathrm{C}$.

\section{Acylated histone H4K16 fusion protein}

Recombinant expression of acylated H4 K16 fusion protein was performed in C321. $\Delta$ A.exp cells, transformed with the appropriate amber mutant vectors, in standard LB medium supplemented with kanamycin and spectinomycin. Cells were inoculated from an overnight culture and incubated at $37^{\circ} \mathrm{C}$ shaking, up to $\mathrm{OD}_{600}>1.0$. The culture was supplemented with $10 \mathrm{mM}$ unnatural amino acid and $20 \mathrm{mM}$ nicotinamide (NAM) and incubated for another 30 min before induction with $0.2 \%$ arabinose. Protein expression was carried out at $37^{\circ} \mathrm{C}$ shaking for $16 \mathrm{~h}$. Cells were harvested and washed with cold PBS containing 20 mM NAM.

\section{Nicotinamidase pncA-MBP}

Expression of the nicotinamidase pncA fused to a maltose binding protein from a pMalc2x-pncA plasmid was performed in E. coli BL21(DE3) cells. Plasmid DNA was transformed into chemical competent BL21(DE3) cells via heat shock and used to inoculate $100 \mathrm{~mL}$ LB medium supplemented with ampicillin and $0.2 \%$ glucose. The preculture was incubated at $37{ }^{\circ} \mathrm{C}$ shaking. Next day, the main culture was inoculated to $\mathrm{OD}_{600} 0.1$ and incubated at $37^{\circ} \mathrm{C}$ shaking. Protein expression was induced at $\mathrm{OD}_{600} 1.0$ with $1 \mathrm{mM}$ IPTG and the cell culture shifted to $20^{\circ} \mathrm{C}$. $6 \mathrm{~h}$ after induction cells were harvested, washed with cold PBS and stored at $-20^{\circ} \mathrm{C}$. 


\subsubsection{Cell disruption}

Frozen cell pellets were thawed on ice and resuspended in lysis buffer. The composition of the lysis buffer depended on the buffer used for purification of the protein afterwards but always contained $2 x$ DTT and $1 x$ Protease inhibitor cocktail (PIC) (2.1.6). After resuspension the cells were decomposed in a cooled pneumatic cell desintegrator (Microfluidizer 110S, Microfluidics, USA) using high pressure (552 kPa). Cell debris and insoluble fractions were removed by centrifugation $\left(4{ }^{\circ} \mathrm{C}, 30 \mathrm{~min}, 20000 \mathrm{rpm}\right)$. The clear supernatant containing the desired protein was further used for purification.

\subsubsection{Protein biochemical methods}

\subsubsection{SDS gel electrophoresis (SDS-PAGE)}

Size analysis of proteins was performed by SDS gel electrophoresis. The gels consist of a stacking gel part with $4 \%$ acrylamide and a separating gel part with $12 \%$ or $15 \%$ acrylamide depending on the size of the proteins (2.1.7). Protein samples were mixed with loading buffer (2.1.6) and applied to the pockets of the SDS gel. For size determination different standards were used (2.1.12). The SDS gel electrophoresis chamber was filled with SDS runing buffer (2.1.6) and a potential of $200 \mathrm{~V}$ applied. The electrophoresis was performed for 60-80 min depending on the protein size and acrylamide concentration of the gel.

\subsubsection{Immunoblot (Western Blot)}

The immunoblot was used for detection of proteins with specific tags. The proteins were separated by size with SDS-PAGE and transfered on a nitrocellulose or polyvinylidene floride membrane. The SDS gel was soaked for $10 \mathrm{~min}$ in transfer buffer (2.1.6) before placing it on top of the membrane. This order applies to the semi-dry electroblotter. For usage of the wet electroblotter the membrane lies on top of the gel. On either side of the 
stack two or three pieces of Whatman paper soaked in transfer buffer were placed before inserting the stack into the electroblotter. In the semi-dry blotter $250 \mathrm{~mA}$ per stack was applied and $100 \mathrm{~V}$. The electrophoresis was performed for $30-45$ min depending on the size of the proteins. When the wet blotter was used the device was placed at $4{ }^{\circ} \mathrm{C}$ and the transfer buffer was precooled. For two stacks $100 \mathrm{~V}$ and $500 \mathrm{~mA}$ were applied for $30 \mathrm{~min}$. After the transfer the membrane was treated with Ponceau S staining solution (2.1.6) to fix the protein on the membrane. Subsequent incubation with buffer solution (Table 2.3) for at least 10 min prevented unspecific binding of the antibody. Incubation with primary antibody solution was performed differently depending on the antibody and is described below. The membrane was rinsed with $\mathrm{dH}_{2} \mathrm{O}$ thoroughly and a solution with the corresponding secondary antibody (2.1.10) in a dilution of 1:10000 applied. After incubation for $1 \mathrm{~h}$ at RT on a shaker the membrane was again thoroughly rinsed with $\mathrm{dH}_{2} \mathrm{O}$ and transferred into PBS or TBS buffer (2.1.6). ECL detection reagents (GE Healthcare, UK) were mixed and applied on the membrane following manufacturer's protocol. Emitting light was captured on hyperfilm ECL (GE Healthcare, UK).

The nitrocellulose transfer membrane could be used without treatment but the polyvinylidene fluoride membrane needed to be activated. It was incubated in $100 \%$ methanol for 1 min followed by hydrophilization in water.

Table 2.3: The different primary antibodies with used concentrations and corresponding buffer solutions. Buffer solutions with anti-histone H4K16ac, anti-crotonyllysine and anti-histone H4 antibody were supplemented with $0.1 \%$ Tween 20 . Also buffer solutions used in the washing step between incubation with primary and secondary antibody contained $0.1 \%$ Tween 20.

\begin{tabular}{ll}
\hline Buffer solution & Antibody \\
\hline PBS $+3 \%$ BSA & anti-his antibody (1:3000) \\
& anti-acetyllysine antibody (1:3000) \\
& anti-histone H4 antibody (1:20000) \\
TBS $+3 \%$ BSA & anti-crotonyllysine antibody (1:4000) \\
TBS $+5 \%$ milk & anti-histone H4K16ac antibody (1:5000) \\
\hline
\end{tabular}




\subsubsection{Protein purification}

Purification of O-acetyl-ADP-ribose synthases (OAADPRS)

Frozen cell pellets were thawed on ice and resuspended in cold Ni-NTA wash buffer (2.1.6) supplemented with lysozyme $(0.2 \mathrm{mg} / \mathrm{mL})$. Lysis was performed using a pneumatic cell disintegrator (2.2.2.4). After removal of cell debris via centrifugation HisPur ${ }^{\mathrm{TM}} \mathrm{Ni}^{2+}$ NTA Resin (2.1.2) (1 mL slurry in $50 \mathrm{~mL}$ solution) was added to the supernatant containing the protein of interest and incubated $1 \mathrm{~h}$ at $4{ }^{\circ} \mathrm{C}$ on a roller. The suspension was loaded on a plastic column (BioRad, München) with a frit and the resin was washed with two column volumes Ni-NTA wash buffer (2.1.6). Elution of the protein was performed using Ni-NTA elution buffer (2.1.6). For determination of OAADPRS containing fractions a SDSPAGE was performed. Fractions corresponding to the size of the protein were pooled, concentrated and loaded on a HiLoad ${ }^{\mathrm{TM}}$ 26/70 Superdex ${ }^{\mathrm{TM}} 200$ size-exclusion chromatography column (GE healthcare, UK) equilibrated in gel filtration buffer (2.1.6). Absorption was monitored at $254 \mathrm{~nm}$ and $4 \mathrm{~mL}$ fractions collected. Fractions containing OAADPRS, verified by SDS-PAGE, were pooled and concentrated in a microfiltrator (Amicon Ultra-15 Centrifugal Filter Unit, $30 \mathrm{kDa}$, Merck Millipore). Subsequently the protein solution was aliquoted, flash frozen in liquid nitrogen and stored at $-80^{\circ} \mathrm{C}$. 


\section{Purification of CobB}

The purification of CobB protein from frozen cell pellets was performed as described for OAADPRS. The corresponding size exclusion chromatogram is shown in Figure 2.1. For concentration a microfiltrator with a $10 \mathrm{kDa}$ molecular weight cut-off was used. Protein solution was aliquoted, flash frozen in liquid nitrogen and stored at $-80^{\circ} \mathrm{C}$.

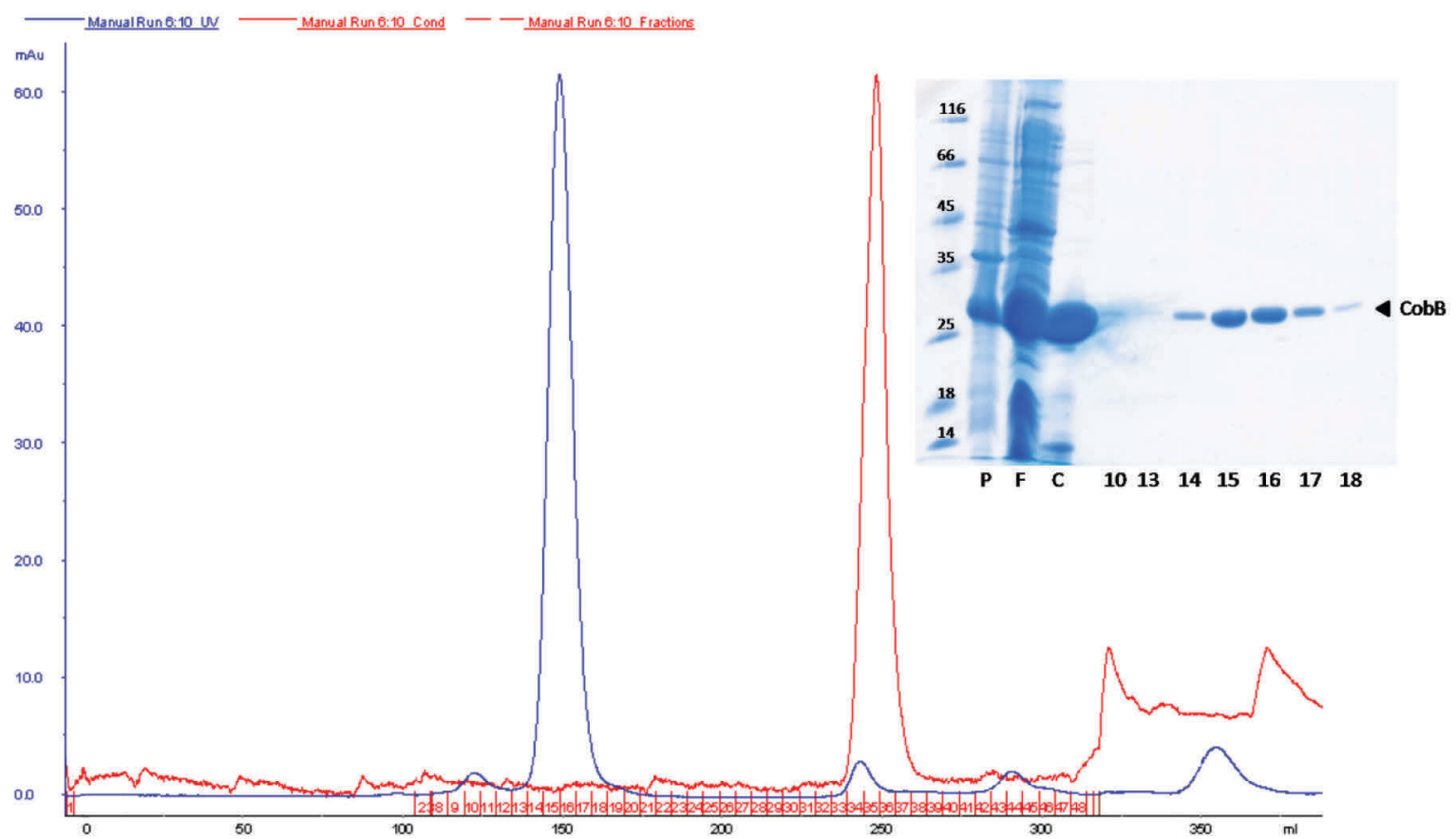

Figure 2.1: Size exclusion chromatogram of affinity tag purified CobB using a Superdex 200 26/70 column.

The synthase eluted with a size of $26 \mathrm{kDa}$. The $12 \%$ SDS gel displays CobB after the hexahistidine tag affinity purification (C) and fractions from the chromatogram. (P: cell pellet, F: flowthrough from hexahistidine tag affinity purification). 


\section{Purification of Sirt1}

The purification of Sirt1 protein from frozen cell pellets was performed as described for OAADPRS. The corresponding size exclusion chromatogram is shown in Figure 2.2. For concentration a microfiltrator with a $10 \mathrm{kDa}$ molecular weight cut-off was used. Protein was stored at $4{ }^{\circ} \mathrm{C}$.

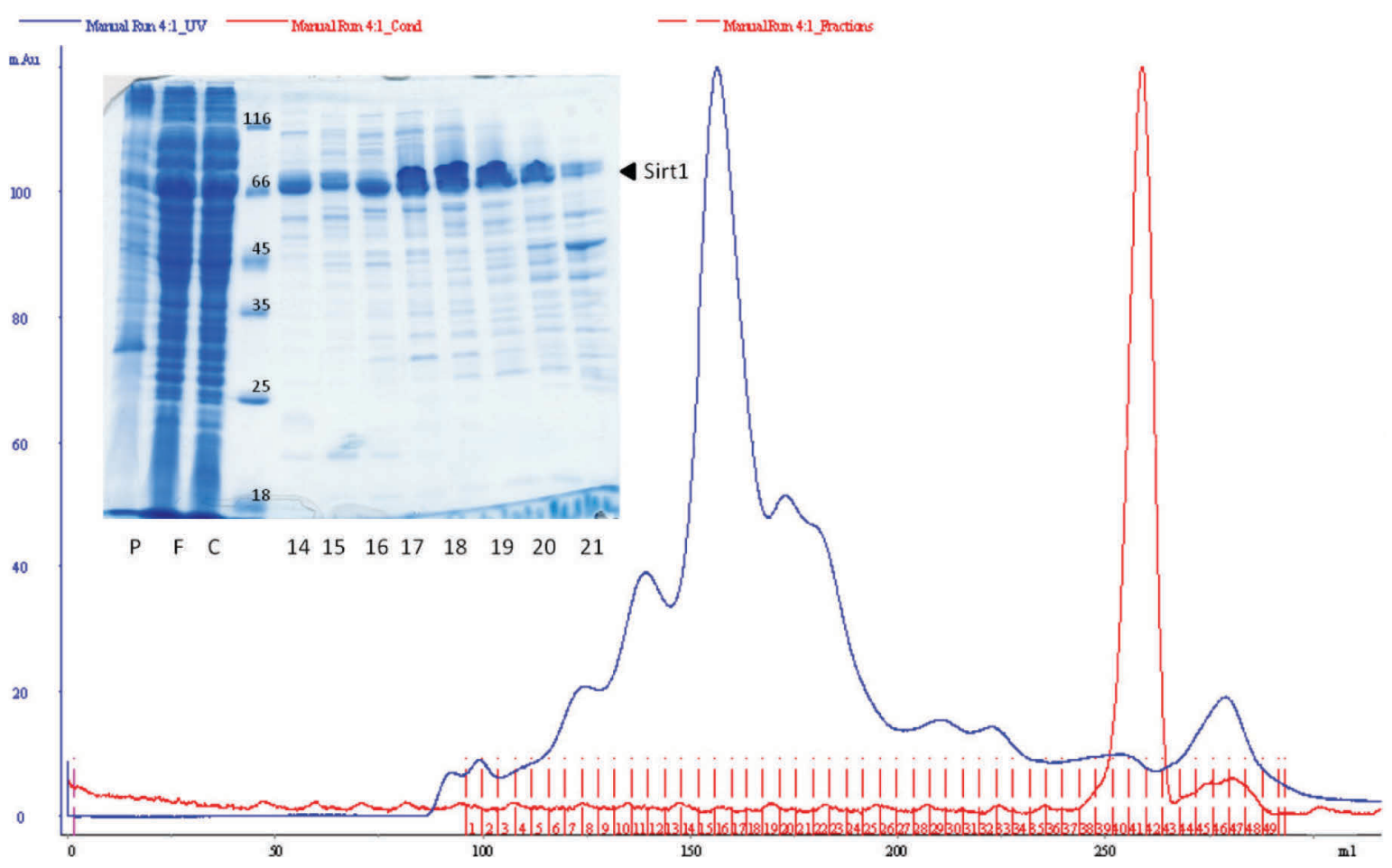

Figure 2.2: Size exclusion chromatogram of affinity tag purified Sirt1 using a Superdex 200 26/70 column. The synthase eluted with a size of $49 \mathrm{kDa}$. The $12 \%$ SDS gel displays Sirt1 after the hexahistidine tag affinity purification $(C)$ and fractions from the chromatogram. (P: cell pellet, F: flowthrough from hexahistidine tag affinity purification).

\section{Purification of acylated histone H4K16 fusion protein}

Frozen cell pellets were thawed on ice, followed by lysis in $30 \mathrm{~mL}$ PBS supplemented with $20 \mathrm{mM}$ NAM, $1 \mathrm{mM}$ DTT, $1 \mathrm{mM}$ PMSF, 1 XPIC and $0.2 \mathrm{mg} / \mathrm{mL}$ lysozyme. The cells were incubated at $37^{\circ} \mathrm{C}$ for 20 min shaking and then lysed via a pneumatic cell desintegrator on 
ice (2.2.2.4). The lysates were clarified by centrifugation ( $15 \mathrm{~min}, 18000 \mathrm{rpm}, 4^{\circ} \mathrm{C}$ ) and washed in PBS supplemented with $20 \mathrm{mM}$ NAM, $1 \mathrm{mM}$ DTT and 1\% Triton X-100. The inclusion bodies were collected as above and washed in PBS with $20 \mathrm{mM}$ NAM, $1 \mathrm{mM}$ DTT. The insoluble fraction was pelleted again, macerated in $1 \mathrm{~mL}$ DMSO and incubated at RT for $30 \mathrm{~min}$. Proteins were extracted from inclusion bodies by adding $30 \mathrm{~mL}$ of $6 \mathrm{M}$ guanidinium chloride, $20 \mathrm{mM}$ Tris ( $\mathrm{pH} 8.0), 2 \mathrm{mM}$ DTT and then incubated at $37^{\circ} \mathrm{C}$ for $1 \mathrm{~h}$ shaking, centrifuged as above and loaded on a pre-equilibrated $\mathrm{Ni}^{2+}-\mathrm{NTA}$ column. The column was washed with $100 \mathrm{~mL} 8 \mathrm{M}$ urea, $100 \mathrm{mM} \mathrm{NaH}_{2} \mathrm{PO}_{4}, 1 \mathrm{mM}$ DTT (pH 6.2). Proteins were eluted with $7 \mathrm{M}$ urea, $20 \mathrm{mM}$ sodium acetate, $200 \mathrm{mM} \mathrm{NaCl}, 1 \mathrm{mM}$ DTT $(\mathrm{pH}$ 4.5). The eluates were dialyzed against $5 \mathrm{mM} \beta$-mercaptoethanol. The protein solution was brought to $50 \mathrm{mM}$ Tris- $\mathrm{HCl}(\mathrm{pH} 7.4)$, supplemented with TEV protease $(20 \mu \mathrm{g} / \mathrm{mL})$ and incubated until cleavage was complete (monitored by SDS-PAGE). Subsequently, salts were removed as above and the protein lyophilized.

\section{Purification of pncA-MBP}

Frozen cell pellets were thawed on ice and resuspended in amylose resuspension buffer (2.1.6). Lysis was performed using a pneumatic cell disintegrator (2.2.2.4). After removal of cell debris via centrifugation the supernatant with the protein of interest was supplemented with amylose resin (2.1.2) ( $2 \mathrm{~mL}$ slurry in $50 \mathrm{~mL}$ solution) and incubated $1 \mathrm{~h}$ at $4{ }^{\circ} \mathrm{C}$. The suspension was loaded on a plastic column (BioRad, München) with a frit and the resin was washed with amylose wash buffer (2.1.6). Elution of the protein was performed using amylose elution buffer (2.1.6). Fractions were run on a $15 \%$ SDS gel to determine purity of the protein. Pnca-MBP containing fractions were pooled, flash frozen in liquid nitrogen and stored at $-80{ }^{\circ} \mathrm{C}$. The activity of pncA was analyzed via an enzymatic coupled assay (2.2.4.1). 


\subsubsection{Size exclusion chromatography}

Since all proteins were temperature sensitive size exclusion chromatography was performed at $4{ }^{\circ} \mathrm{C}$. At first the chromatography column was equilibrated with gel filtration buffer (2.1.6) until the absorption was stable. The protein solution was sterile filtered, applied to the column and the size separation performed at a flow rate of $1.5 \mathrm{~mL} / \mathrm{min}$. The eluent was fractionated in $4 \mathrm{~mL}$ volumes and the containing protein visualized on a SDS gel. Fractions containing the protein of interest were pooled and concentrated by microfiltration to $\sim 1 \mathrm{mg} / \mathrm{mL}$. Aliquots of OAADPRS and CobB were flash frozen and stored at $-80{ }^{\circ} \mathrm{C}$. Sirt1 protein was stored at $4{ }^{\circ} \mathrm{C}$.

\subsubsection{Spectroscopic methods and assays}

\subsubsection{Enzymatic coupled sirtuin assay}

Since the reaction products of the sirtuin catalyzed reaction are not photometrically traceable, an enzymatic coupled assay was used to determine the activity of the synthase. The depicted assay in Figure 2.3 continuously measures nicotinamide formation by using a coupled enzyme system with the nicotinamidase pncA and glutamate dehydrogenase. The pncA hydrolyzes nicotinamide to nicotinic acid and ammonia. Ammonia, together with $\alpha$-ketoglutarate and NADPH are converted to glutamate and $\mathrm{NADP}^{+}$by glutamate dehydrogenase. Amounts of NADPH can be spectrophotometrically measured at a wavelength of $340 \mathrm{~nm}$. The reaction mixture contained:

\begin{tabular}{rll}
\hline 3.3 & $\mathrm{mM}$ & $\alpha$-ketoglutarate \\
0.2 & $\mathrm{mM}$ & NADPH \\
7.5 & $\mathrm{U}$ & glutamate dehydrogenase \\
1 & $\mu \mathrm{M}$ & pncA \\
0.5 & $\mu \mathrm{M}$ & synthase \\
0.75 & $\mathrm{mM}$ & NAD $^{+}$ \\
2.5 & $\mathrm{mM}$ & acetyl-CoA \\
\hline
\end{tabular}


All components except $\mathrm{NAD}^{+}$and acetyl-CoA were mixed in gel filtration buffer and equilibrated until a stable absorption at $340 \mathrm{~nm}$ was obtained. $\mathrm{NAD}^{+}$was added and the mixture equilibrated again. To start the reaction cycle acetyl-CoA was added and the absorption measured over $10 \mathrm{~min}$. Kinetic parameters could be calculated from the absorption decrease.
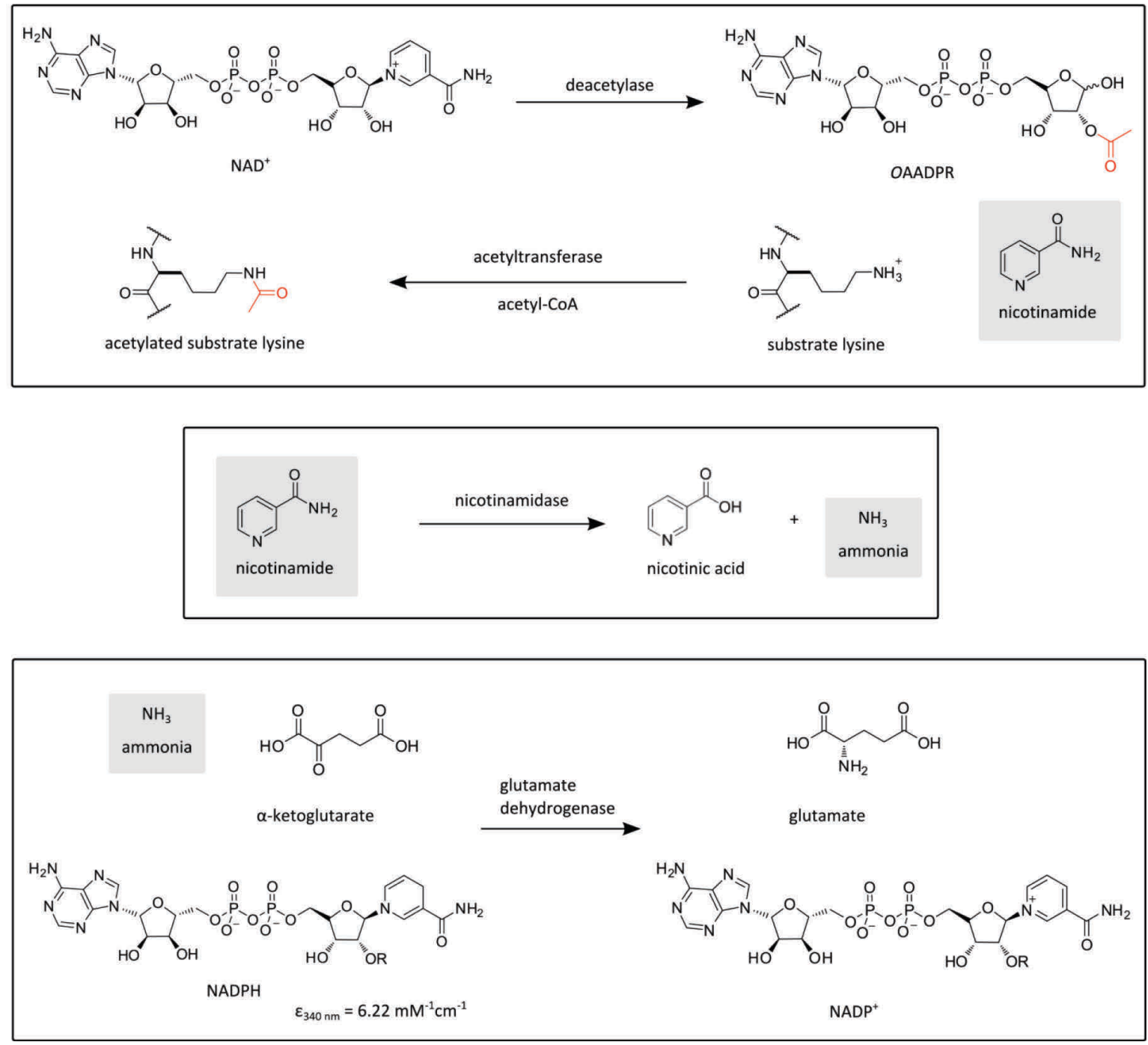

Figure 2.3: Enzymatic coupled sirtuin assay. ${ }^{\text {modified from } 59}$ An acetyltransferase loads the lysine residue with an acetyl group from acetyl-CoA. Nicotinamide is cleaved off $\mathrm{NAD}^{+}$and the acetyl group transferred from the substrate lysine to the remaining ADP-ribose. The deacetylated lysine residue can be loaded again by the acetyltransferase. Nicotinamide is converted to nicotinic acid and ammonium whereas ammonium serves as a substrate in the photometrically traceable reaction. NADPH is oxidized to NADP ${ }^{+}$by glutamate dehydrogenase while ammonia and $\alpha$-ketoglutarate are converted to glutamate. ${ }^{58}$ The absorption maximum of NADPH lies at $340 \mathrm{~nm}$. 


\subsubsection{Resazurin-Assay}

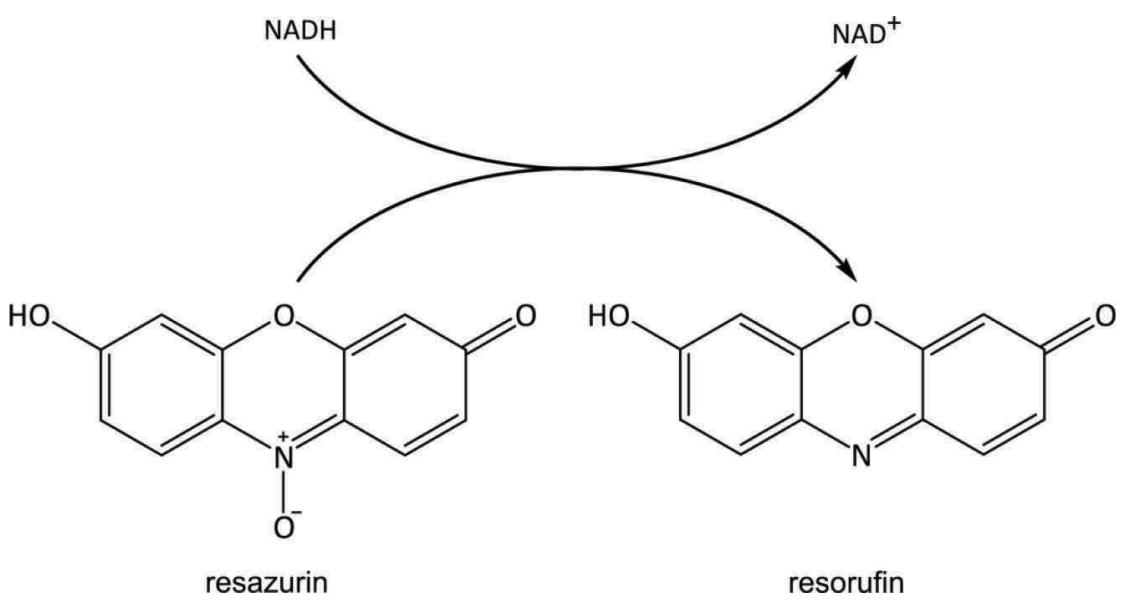

Figure 2.4: Reaction of resazurin (7-hydroxy-10-oxidophenoxazin-10-ium-3-one) to resorufin by acceptance of electrons from NADH which is oxidized to NAD ${ }^{+}$.

Resazurin is a blue redox dye which exhibits fluorometric change due to cellular metabolic activity. Intracellularly it is reduced to resorufin, a strong fluorophore, by oxidation of NADH, FADH, FMNH or NADPH while resazurin itself is only weakly fluorescent (Figure 2.4). The fluorescence signal of resorufin can be detected by application of $540 \mathrm{~nm}$ excitation wavelength and $590 \mathrm{~nm}$ emission wavelength. The redox dye is stable in culture media, non-toxic to cells and its reduction is directly dependent on the number of viable cells.

For determination of the metabolic activity of cells samples were taken at different time points during protein expression. The optical density of the samples was normalized and $200 \mu \mathrm{L}$ suspension was added to $10 \mu \mathrm{M}$ resazurin sodium salt (Sigma-Aldrich, Steinheim) in a 96-well microplate. Fluorescence was measure over $30 \mathrm{~min}$ in the FLUOstar plate reader and the percentage of the samples' metabolic activity was calculated. Values were compared to a control whose values were set to $100 \%$. 


\subsubsection{NAD ${ }^{+}$-Assay}

The EnzyChrom ${ }^{\text {TM }} \mathrm{NAD}^{+} / \mathrm{NADH}$ Assay Kit (2.1.3) was used to determine $\mathrm{NAD}^{+}$ concentration in cells. The colorimetric reaction is based on the reduction of a formazan reagent by $\mathrm{NADH}$ which is formed in a lactate dehydrogenase cycling reaction from $\mathrm{NAD}^{+}$ present in the cell. The absorption of the reduced reagent can be detected at $565 \mathrm{~nm}$ and is proportional to the $\mathrm{NAD}^{+}$concentration in the sample.

Cell samples were taken $2 \mathrm{~h}$ after induction and the optical density normalized. The assay was performed following the manufacturer's protocol using $\mathrm{NAD}^{+}$extraction buffer as first reagent in the sample preparation for $\mathrm{NAD}^{+}$determination.

\subsubsection{Activity test of pncA-MBP}

For activity determination of purified nicotinamidase pncA-MBP an assay was performed based on the enzymatic coupled sirtuin assay (2.2.4.1). In this assay nicotinamide is converted to nicotinic acid and ammonium by pncA-MBP (Figure 2.5). The ammonium serves as a substrate in the reaction where NADPH is oxidized to NADP ${ }^{+}$. The decrease of the amount of NADPH during the experiment can be detected at a wavelength of $340 \mathrm{~nm}$.
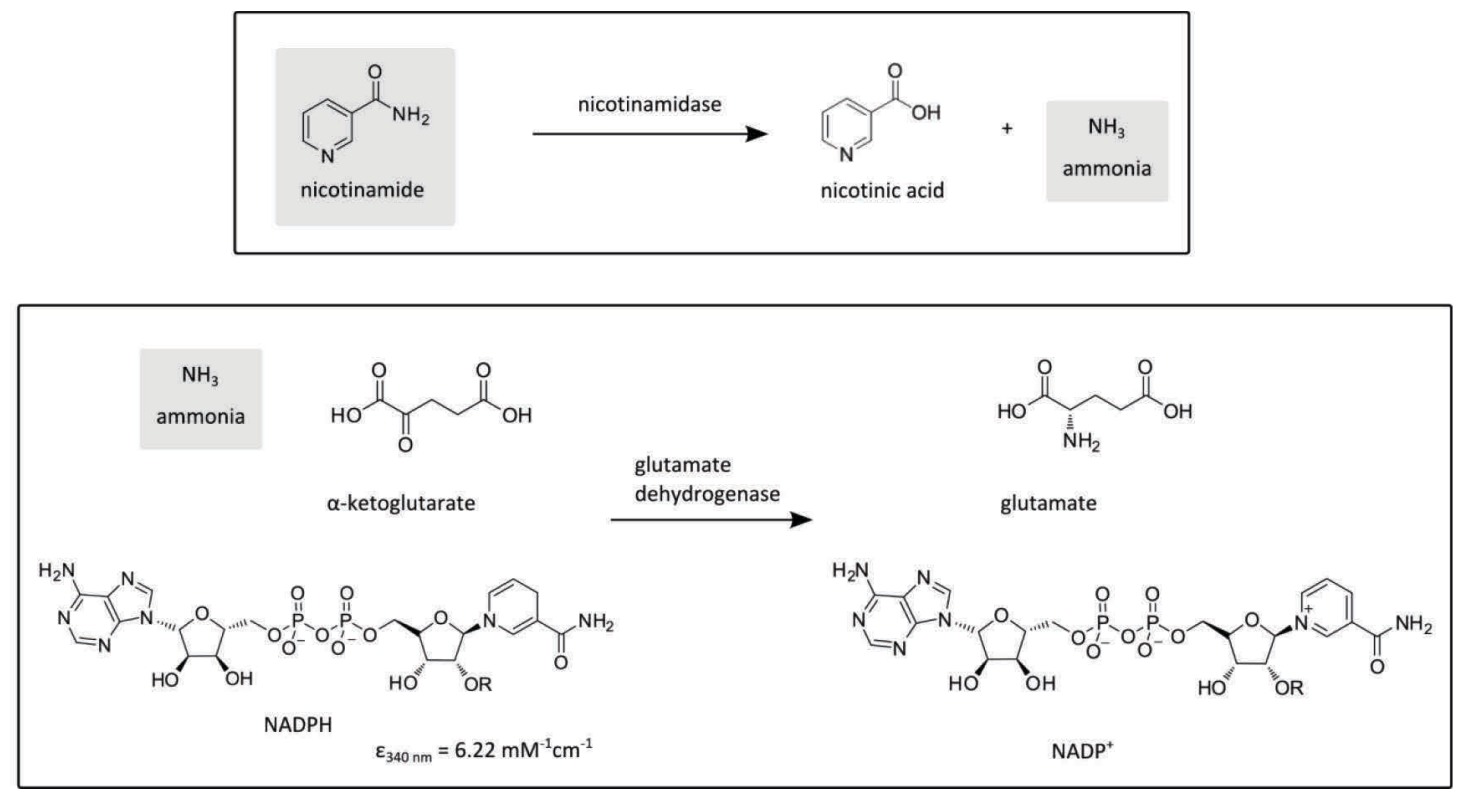

Figure 2.5: Enzymatic coupled assay for analysis of pncA-MBP activity. 
The reaction mixture contained:

\begin{aligned} & \hline $50 \mathrm{mM} \mathrm{KP}_{\mathrm{i}} \mathrm{pH} 7,5 \\ & 3.3 \mathrm{mM} \alpha$-ketoglutarate \\ & $200 \mu \mathrm{M}$ NADPH \\ & $1 \mathrm{mM}$ DTT \\ & $30 \mathrm{U} / \mathrm{mL}$ glutamate dehydrogenase \\ & $2 \mu \mathrm{M}$ pncA-MBP \\ & $0.4 \mathrm{mM} \mathrm{MgCl}_{2} \\ &$\hline\end{aligned}

All components except of nicotinamide were mixed and equilibrated until a stable absorption at $340 \mathrm{~nm}$ was obtained. Different amounts of nicotinamide, ranging from $0.01 \mathrm{mM}$ to $1 \mathrm{mM}$, were added to the reaction mixture and the absorption measured over $10 \mathrm{~min}$. From absorption decrease the velocity of the reaction could be calculated.

\subsubsection{Lysine deacylation assay}

For determination of the ability of CobB deacetylase to cleave different acylations at histone H4K16 a lysine deacylation assay was established. $30 \mathrm{ng} / \mu \mathrm{L}$ modified histone $\mathrm{H} 4$ in reaction buffer (500 mM Tris pH 7.5, $140 \mathrm{mM} \mathrm{NaCl}, 20 \mathrm{mM} \mathrm{Mg}(\mathrm{OAc})_{2}$ and $80 \mu \mathrm{M} \mathrm{NAD}^{+}$) were mixed with $2 \mathrm{ng} / \mu \mathrm{L}$ CobB deacetylase (30:1 molar ratio) and incubated at RT. After $0,1,2,3,4,5,10,15,20$ and $30 \mathrm{~min} 7.5 \mu \mathrm{L}$ samples were taken and quenched by addition of preheated $2.5 \mu \mathrm{l} 4 \times$ SDS-PAGE sample buffer containing $48 \mathrm{mM}$ NAM. The samples were incubated for $5 \mathrm{~min}$ at $95^{\circ} \mathrm{C}$ and analyzed by SDS-PAGE using $15 \%$ gels and immunoblotting (2.2.3). Polyclonal Histone H4K16ac antibody (2.1.10) was used to detect acetylation, butyrylation and propionylation and an anti-crotonyllysine antibody (2.1.10) was used to detect H4K16 crotonylation. Subsequently, the membrane was stripped and anti-histone $\mathrm{H} 4$ antibody (2.1.10) used to detect bulk histone H4. Quantification of the acylation cleavage was performed by comparison of acylation signals to amount of bulk histone applied using the computer program ImageJ (NIH, USA). 


\subsubsection{One-step synthesis of the metabolite $O$-acetyl-ADP-ribose}

The first part of the synthesis was performed in a $2 \mathrm{~mL}$ micro tube. $400 \mu \mathrm{L}$ glacial acetic acid were added to $16 \mathrm{mg}(0.15 \mathrm{mmol})$ sodium carbonate and the mixture stirred at RT. After the sodium carbonate was dissolved $50 \mathrm{mg}(0.075 \mathrm{mmol}) \mathrm{NAD}^{+}$was added. The micro tube was heated to $90{ }^{\circ} \mathrm{C}$ in a thermomixer for 20 min until the $\mathrm{NAD}^{+}$was completely dissolved. $1.0 \mathrm{~mL}$ of denatured alcohol (90:5:5 [v/v/v] ethanol/ methanol/ 2propanol) was slowly added to the mixture to dilute residual sodium carbonate. After transfer into a $15 \mathrm{~mL}$ falcontube the product was precipitated by rapid addition of another $2.6 \mathrm{~mL}$ of denatured alcohol. The suspension was centrifuged (RT, $20 \mathrm{~min}$, $4000 \mathrm{rpm})$, the supernatant removed and the remaining light tan solid dissolved in water for purification.

As first purification step an anion exchange chromatography was performed using a highpressure liquid chromatography (HPLC) system. A prepacked aminopropyl-linked silica gel column (Macherey-Nagel, Dülmen) was washed with elution buffer several times before usage. The elution buffer consisted of two solutions. Solution A contained $1 \mathrm{M}$ acetic acid and solution $\mathrm{B}$ contained $1 \mathrm{M}$ acetic acid and $1 \mathrm{M}$ sodium acetate. The column was equilibrated in $90 \%$ solvent $A$ and $10 \%$ solvent B prior to the chromatography run. After injection of the sample following separation gradient was applied:

\begin{tabular}{ccc}
\hline Time [min] & Solvent A & Solvent B \\
\hline 0 & 90 & 10 \\
5 & 90 & 10 \\
10 & 50 & 50 \\
20 & 50 & 50 \\
25 & 0 & 100 \\
30 & 0 & 100 \\
\hline
\end{tabular}

Absorption at $254 \mathrm{~nm}$ was monitored, substance containing fractions collected and the solvents evaporated. For removal of residual sodium acetate three desalting cycles had to be performed before the samples could be analyzed by mass spectrometry. Each sample 
was dissolved in $50 \mu \mathrm{L}$ water and slowly diluted with denatured alcohol (90:5:5 [v/v/v] ethanol/ methanol/ 2-propanol) to a final concentration of 95:5 (v/v) alcohol/ water. The product precipitated as a white solid. The suspension was centrifuged (RT, $20 \mathrm{~min}, 4000$ rpm) and the supernatant removed. After another two desalting cycles the precipitate was dissolved again in water and applied to the last purification step, the hydrophilic interaction chromatography (HILIC). A polyHYDROXYETHYL $A^{\mathrm{TM}}$ column was washed several times with elution buffer before the chromatography run was performed. Solutions for the elution buffer were $10 \mathrm{mM}$ ammonium acetate as solvent $A$ and acetonitrile as solvent $\mathrm{B}$. The following gradient was used to separate the products:

\begin{tabular}{ccc}
\hline Time [min] & Solvent A & Solvent B \\
\hline 0 & 20 & 80 \\
15 & 20 & 80 \\
35 & 80 & 20 \\
55 & 100 & 0 \\
60 & 100 & 0 \\
65 & 20 & 80 \\
\hline
\end{tabular}

Absorption at $254 \mathrm{~nm}$ was monitored and substance containing fractions collected. The analysis for OAADPR content was performed by mass spectrometry after the solvents were evaporated. Samples containing product were lyophilized and stored at $-20{ }^{\circ} \mathrm{C}$. 


\section{Results}

\subsection{The $O$-acetyl-ADP-ribose synthase (OAADPRS)}

\subsubsection{Engineering and functional optimization of the $O$-acetyl-ADP-ribose synthase}

For the successful engineering of an OAADPR synthase with a functional turnover the individual parts needed to be chosen with care. An orientation for this was provided by parts which can be found in nature. Under physiological conditions the acetylation of lysine substrates is catalyzed by a lysine acetyltransferase and the formation of OAADPR by a class III deacetylase, a sirtuin. Hence, a sirtuin was selected as deacetylase moiety in the synthase. The bacterial sirtuin CobB became the protein of choice because it is stably expressed in E. coli cells and its crystal structure is known. In later investigations of OAADPR functions in eukaryotic cells, it would provide advantages to use a bacterial deacetylase. Finding specific interaction partners and substrats in those systems would be highly limited. CobB is a yeast Sir2 homologue and was originally found to play a role in the cabalamin synthesis of the bacterium Salmonella typhimurium LT2. It partially compensates the inactivation of the phosphoribosyltransferase CobT and was therefore thought to have phosphoribosyltransferase activity. ${ }^{60}$ In Salmonella enterica, however, CobB was found to activate the acetyl-CoA synthetase by hydrolysis of the acetyl group from an acetyllysine in the active site, which revealed the deacetylase function of CobB. ${ }^{61,62}$ CobB from E. coli could be crystallized in complex with an acetyllysine containing peptide from histone H4. Following binding studies with cognate and noncognate peptide substrates revealed only little discrimination between them. Surprisingly binding with the non-cognate histone $\mathrm{H} 4$ peptide showed the lowest dissociation constant while the highest dissociation constant was obtained with a cognate acetyllysinetransferase peptide. ${ }^{63}$ Also the structure of yeast Hst2 (homologue of $\underline{\text { Sir two }}$ 2) had been analyzed in complex with the same acetylated histone $\mathrm{H} 4$ peptide. ${ }^{64}$ Hence, a histone $\mathrm{H} 4$ peptide sequence seemed to be the best choice to harbour the substrate 
lysine in the synthase. But this substrate peptide proved not to be accessible for CobB in the construct with a fused acetyltranferase. In earlier experiments, a construct containing the coding sequence of histone $\mathrm{H} 3$ instead of $\mathrm{H} 4$ showed promising deacetylating activity, but only weak acetylation activity. Therefore, a part of the H3 coding sequence was placed in front of the $\mathrm{H} 4$ sequence. This new peptide linker proved to function as substrate for both enzymatic parts.

As acetyltransferase moiety Hat1 was chosen out of different yeast variants. This choice was based on the stable expression in E. coli compared to constructs with other acetyltransferases. This B-type yeast protein was found to reside in the cytosol of yeast cells but might also be active in the nucleus. ${ }^{65-70}$ Hat1 possibly participates in DNA repair as well as in gene silencing through its acetylation activity during localized chromatin assembly. ${ }^{71}$ In vitro experiments showed specific acetylation of newly synthesized nonnucleosomal $\mathrm{H} 4$ at lysine 5 and 12 . Acetylation of lysine 12 by Hat1 was also demonstrated in vivo at sites of DNA double-strand breaks ${ }^{72}$ and is the most readily acetylated position. Newly synthesized histone $\mathrm{H} 3$ is acetylated as well but in a less conserved pattern. ${ }^{73}$ Therefore, lysine 12 of histone $\mathrm{H} 4$ was determined as substrate lysine in the peptide linker of the synthase.

A scheme of the final engineered $O$-acetyl-ADP-ribose synthase (OAADPRS) is depicted in Figure 3.1. At the N-terminal part of the synthase lies the deacetylase CobB. It consists of two domains connected by four loops. ${ }^{63}$ The large domain forms a classic pyridine dinucleotide binding fold, the Rossmann fold, which is a common feature of NAD ${ }^{+}$-binding domains. $^{74}$ The small domain is composed of two modules. The zinc-binding module contains a structural zinc ion coordinated by four invariant cysteins. The second module is a helical motif including a flexible loop. The binding sites for $\mathrm{NAD}^{+}$and the acetyllysine substrate are located in a cleft between the large and small domain. ${ }^{75}$

The C-terminal part of the synthase forms the acetyltransferase Hat1. It contains two domains connected by an extended loop where a hydrophobic interface is formed. The C-terminal domain of Hat1 contains the acetyl-CoA binding site. This binding cleft lies on the concave surface towards the center of the protein. ${ }^{76}$ The CobB and Hat1 enzymatic 
moieties are linked by a histone $\mathrm{H} 3 / \mathrm{H} 4$ peptide chain. The $\mathrm{H} 4$ sequence harbours the substrate lysine at position 12 .

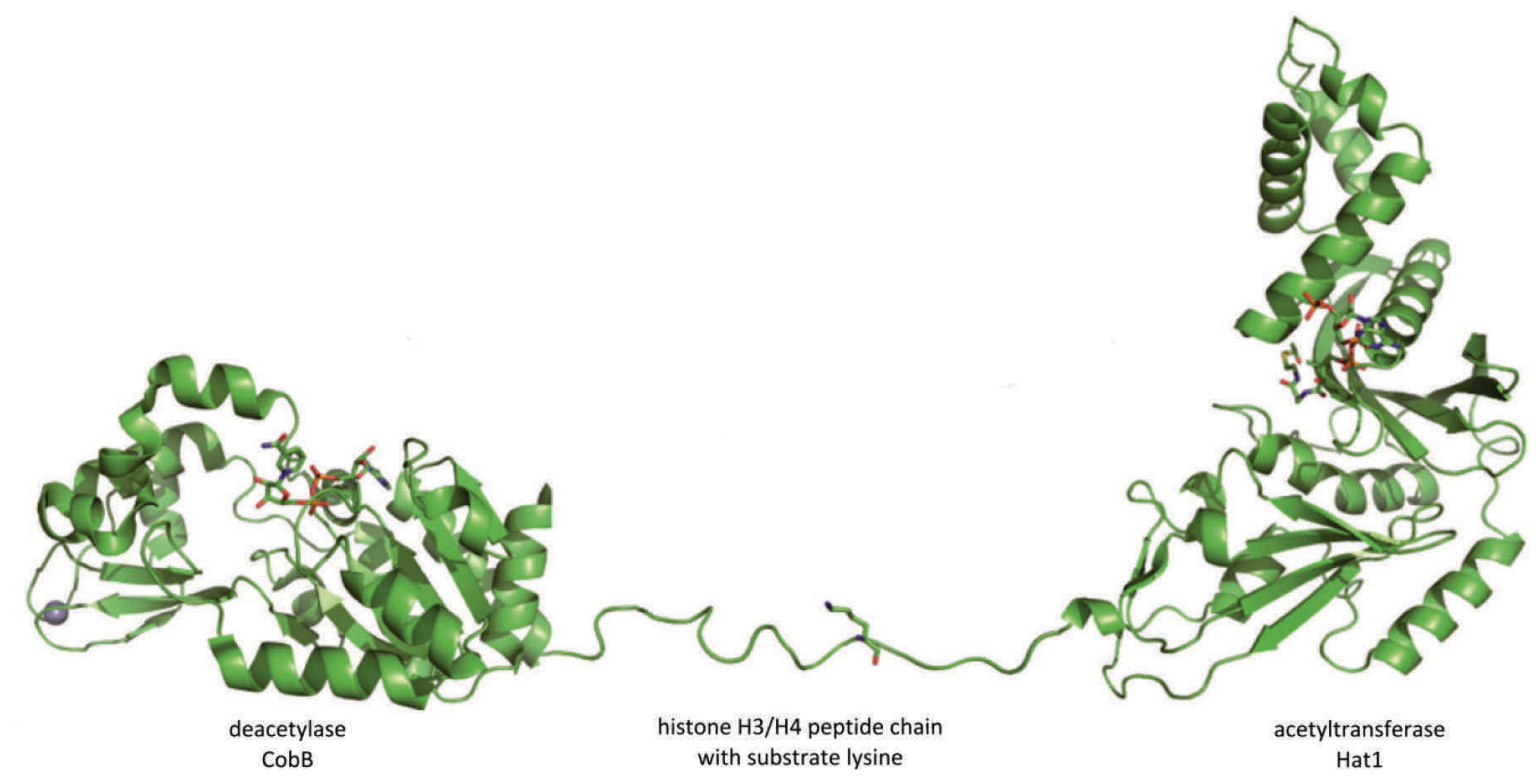

Figure 3.1: Scheme of the $O$-acetyl-ADP-ribose synthase (OAADPR) describing its individual parts. The histone $\mathrm{H} 3 / \mathrm{H} 4$ peptide chain harbours the substrate lysine and links the catalytic core domain of the deacetylase CobB (PDB-ID: 1S5P ${ }^{62}$ ) on the left with the domain of the acetyltransferase Hat1 (PDB-ID: $1 \mathrm{BOB}^{75}$ ) on the right. In the binding cleft of $\mathrm{CobB}$ its co-substrate $\mathrm{NAD}^{+}$is depicted and a structural zinc ion in the zinc-binding module. The substrate of Hat1, acetyl-CoA, is bound in a cavity on the surface of the Hat1 core domain. The structure was composed using PyMOL (DeLano Scientific LLC/Schrödinger LLC, USA).

The function of the OAADPR synthase and the components of the catalytic reaction to produce the metabolite OAADPR are depicted in Figure 3.2. In the first step CobB interacts with the acetylated lysine at position 12 of histone $\mathrm{H} 4$ in the linker peptide and a $\mathrm{NAD}^{+}$molecule. The acetyl group is transferred from the substrate lysine to $\mathrm{C}^{\prime}$ of the nicotinamide ribose of $\mathrm{NAD}^{+}$and nicotinamide is released. After some intramolecular reactions the acetyl group finally binds to the 2'-position of the nicotinamide ribose, forming the metabolite $O$-acetyl-ADP-ribose. In the last step of the reaction cycle a new acetyl group is transferred from an acetyl-CoA molecule to the substrate lysine by Hat1 and the next reaction cycle starts. 


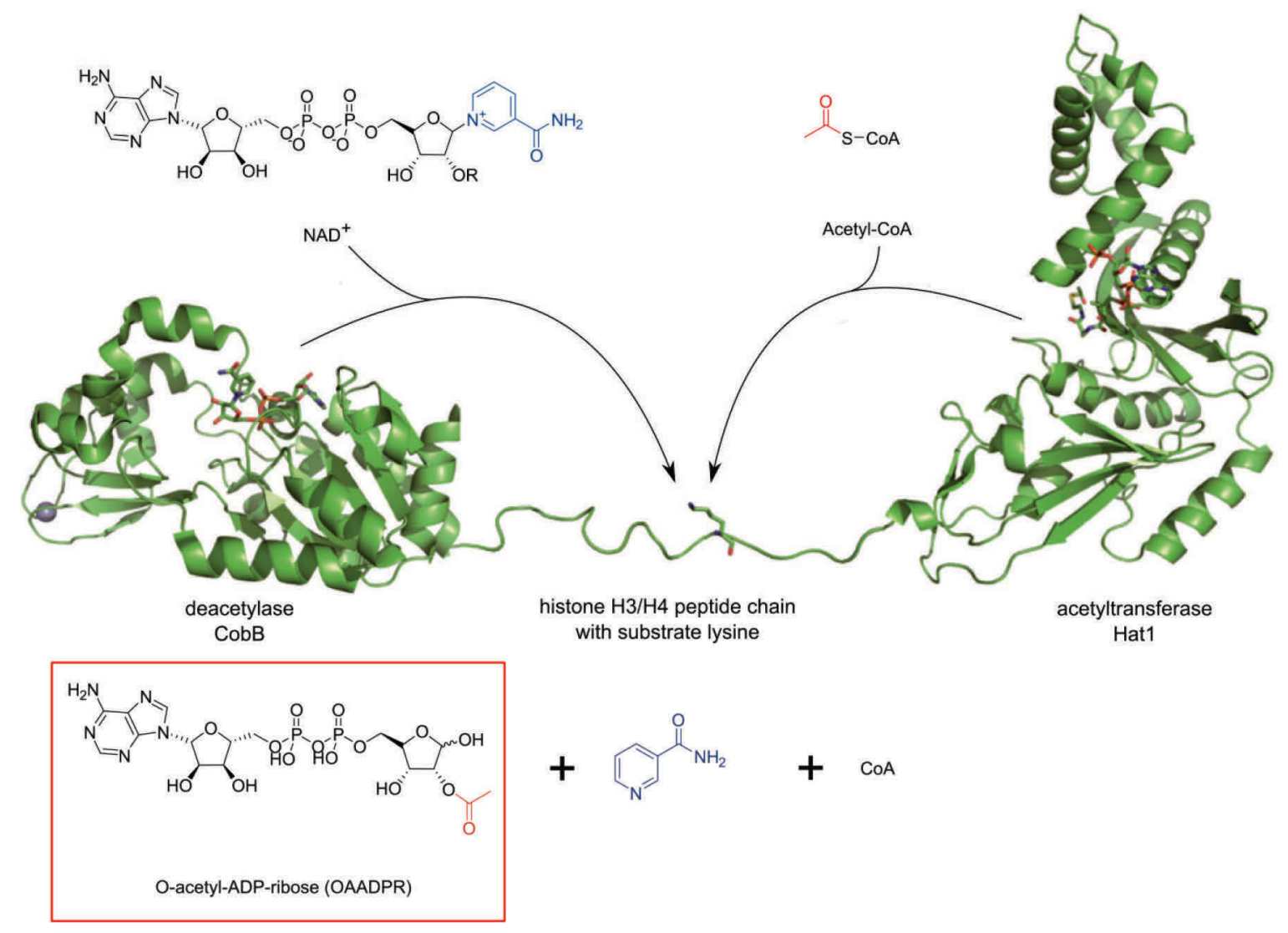

Figure 3.2: Function of the OAADPR synthase and formation of the metabolite OAADPR. Following the release of nicotinamide, CobB relocates the acetyl group from the substate lysine to $\mathrm{NAD}^{+}$. There it finally binds to $\mathrm{C2}^{\prime}$ of the nicotinamide ribose forming OAADPR. At last the acetyltransferase Hat1 reacetylates the substrate lysine by transfer of an acetyl group from acetyl-CoA. Products of one reaction cycle are one molecule of OAADPR, nicotinamide and CoA, respectively.

The first version of the $O$-acetyl-ADP-ribose synthase was named synthase 1.0. For simplyfication the $O$-acetyl-ADP-ribose synthases will be abbreviated "synthases" with the version number behind it. The DNA sequence of synthase 1.0 contained the catalytic core domain (aa 38 -279) of CobB, amplified by PCR from E. coli DH10B cells, and the acetyltransferase Hat1 moiety (aa $1-320$ ) which was amplified from Saccharomyces cerevisiae. The linking histone H3/H4 peptide consisted of amino acids 2 - 35 from histone $\mathrm{H} 3$ and amino acids 8 - 16 from histone $\mathrm{H} 4$. Histone $\mathrm{H} 4$ contained the substrate lysine for both enzymatic moieties at position 12 . The peptide sequences were amplified from 
Xenopus laevis and all components cloned into BamHI and Xhol restriction sites of a pCDF Duet-1 vector. The synthase 1.0 was expressed in E. coli BL21(DE3) cells (2.2.2.3) and purified via a N-terminal hexahistidine tag using Ni-NTA resin. After affinity tag purification, a size exclusion chromatography was performed to remove protein truncations and yield pure protein (2.2.3.3, Figure 3.3). Fractions from the chromatography were analyzed by SDS PAGE. On the gel fractions 18 to 24 contained larger amounts of synthase 1.0 with small impurities (Figure 3.4). The impurity mainly consists of a truncated CobB part of the synthase that can also be seen in fractions 26 and 28.

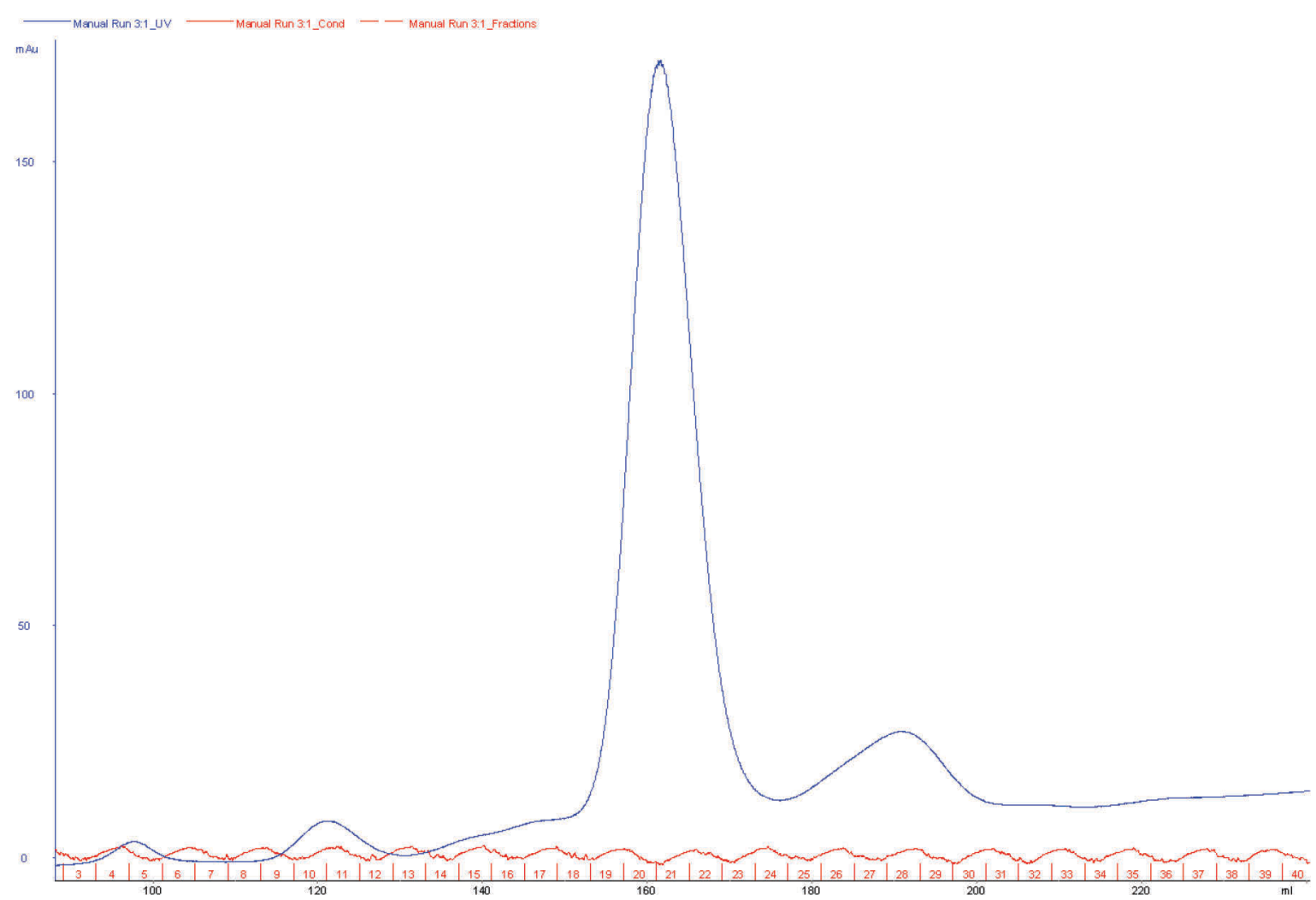

Figure 3.3: Size exclusion chromatogram of the affinity tag purified synthase 1.0 using a Superdex 200 26/70 column. The synthase eluted with a size of $70 \mathrm{kDa}$ after $162 \mathrm{~min}$. After $191 \mathrm{~min}$ CobB truncation of the synthase eluted from the column. 


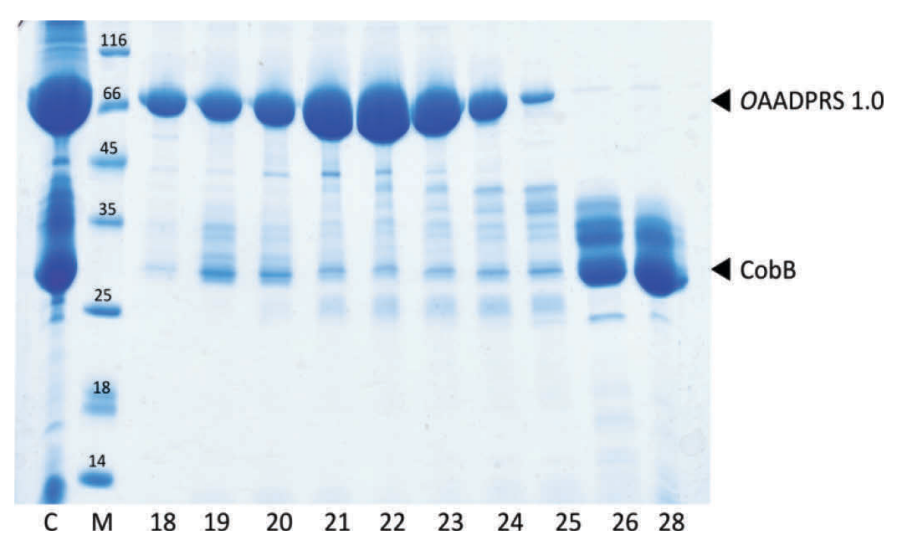

Figure 3.4: $12 \%$ SDS gel showing OAADPRS 1.0 after hexahistidine tag affinity purification (C) and fractions from the size exclusion chromatography (Figure 3.3). Bands at $70 \mathrm{kDa}$ represent the full length protein and at $28 \mathrm{kDa}$ CobB truncations.

Initial activity tests of synthase 1.0 were performed using thin-layer chromatography with a solvent mixture of ethanol and ammonium acetate (ratio 70:30). Since $\mathrm{NAD}^{+}$and OAADPR differ in their solubility in this solvent, an additional spot for OAADPR should be visible on the plate after the run if the synthase was active. The reaction mixture contained the substrates of CobB and Hat1, namely NAD ${ }^{+}$and acetyl-CoA together with nicotinamidase and the synthase 1.0. Nicotinamidase is necessary to hydrolyze nicotinamide, which is a product besides OAADPR of the deacetylation and can inhibit the reaction when aggregated. Samples of the reaction mixture after a certain incubation time were spotted on a TLC plate and the components chromatographically separated. Spots likely containing OAADPR were scraped off the plate and analyzed by mass spectrometry. It was found out that the amount of material in the samples was not sufficient and no traces of OAADPR could be detected.

An alternative method to test the activity of the engineered synthase was the enzymatic coupled sirtuin assay (2.2.4.1), published by Smith et al. in $2009 .{ }^{59}$ Since the reaction products of the deacetylation themselves were not spectrometrically detectable, the produced nicotinamid was coupled to the oxidation of NADPH. NADPH has an absorption maximum at $340 \mathrm{~nm}$ and can therefore be spectrometrically detected. Nicotinamide is converted to nicotinic acid and ammonia by the nicotinamidase pncA. The ammonia is 
finally utilized in the oxidation of NADPH to $\mathrm{NADP}^{+}$by glutamate dehydrogenase (Figure 3.5). The absorption of NADPH was measured during the deacetylation with a spectrophotometer and plotted against time.
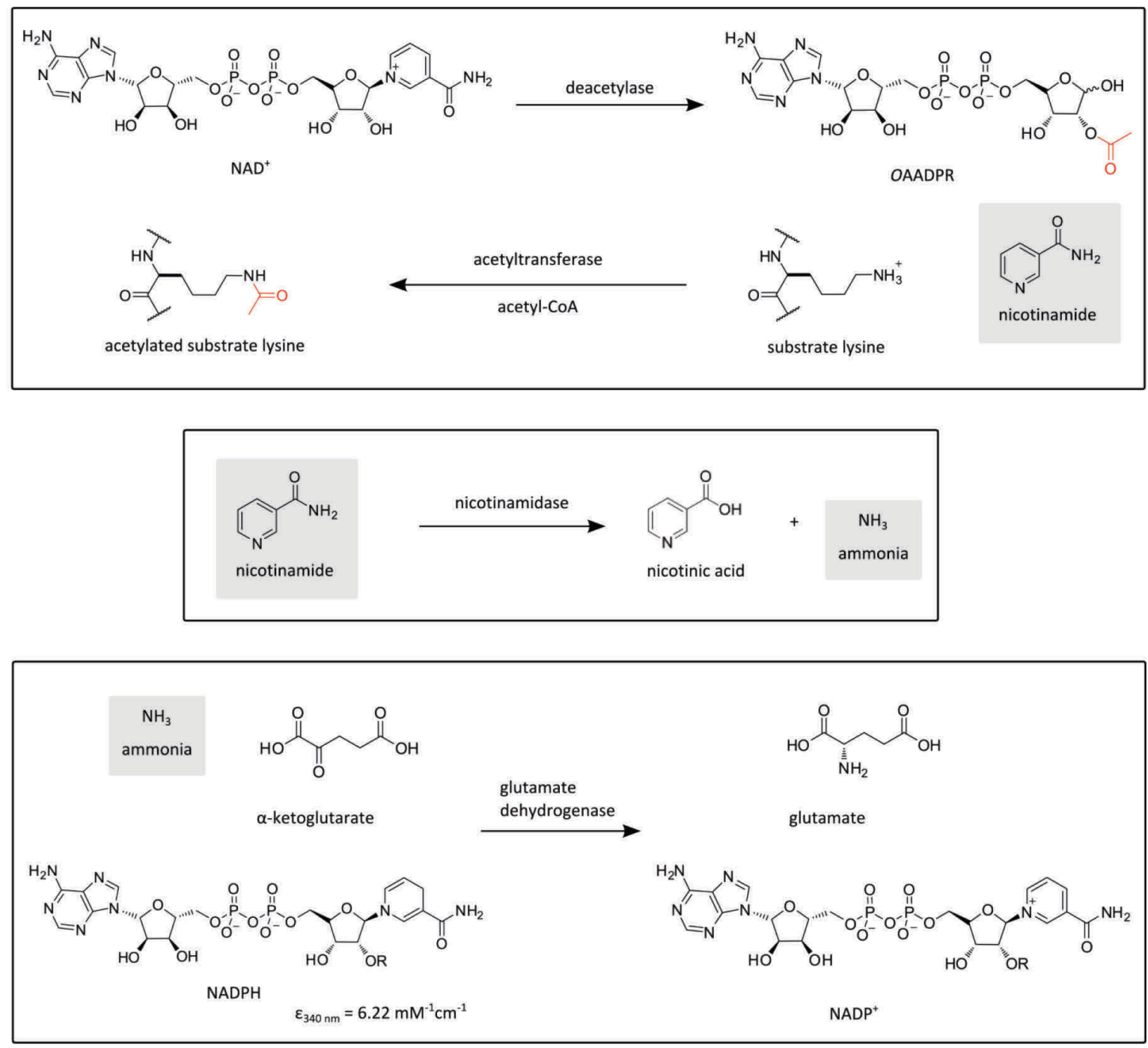

Figure 3.5: Scheme of the enzymatic coupled sirtuin assay. ${ }^{\text {modified from } 59}$ Nicotinamide is cleaved from NAD $^{+}$ and the acetyl group is transferred from the substrate lysine to the remaining ADP-ribose structure of NAD to form OAADPR. The deacetylated lysine residue is charged again with an acetyl group by the acetyltransferase. Nicotinamide is converted to nicotinic acid and ammonium, whereas ammonium serves as a substrate in the photometrically detectable reaction. Then, NADPH is oxidized to NADP ${ }^{+}$by glutamate dehydrogenase while ammonia and $\alpha$-ketoglutarate are converted to glutamate. The absorption maximum of NADPH lies at $340 \mathrm{~nm}$. 
Figure 3.6 details the change in absorption during the reaction catalyzed by synthase 1.0. First, the absorption of the reaction mixture without $\mathrm{NAD}^{+}$and acetyl-CoA was equilibrated. Only slight changes in absorption were visible due to equilibration reactions. Addition of $\mathrm{NAD}^{+}$resulted in a decrease of absorption. One reason for this could be an impurity in the $\mathrm{NAD}^{+}$solution. Aqueous solutions of $\mathrm{NAD}^{+}$are prone to degradation at certain $\mathrm{pH}$ and temperature conditions and they are sensitive to light. Therefore, nicotinamide could have been formed, which would lead to a conversion of NADPH to $\mathrm{NADP}^{+}$and a decrease of the abosorption after addition to the reaction mixture. After a stable absorption was obtained, acetyl-CoA was added. The measurement showed a linear descent of the absorption that referred to a reduction of NADPH. Consequently, the decreasing amount of NADPH led to the conclusion that the synthase was active and catalyzing the turnover of $\mathrm{NAD}^{+}$. The same experiment peformed with buffer instead of synthase showed no significant decrease of the absorption after addition of acetyl-CoA.

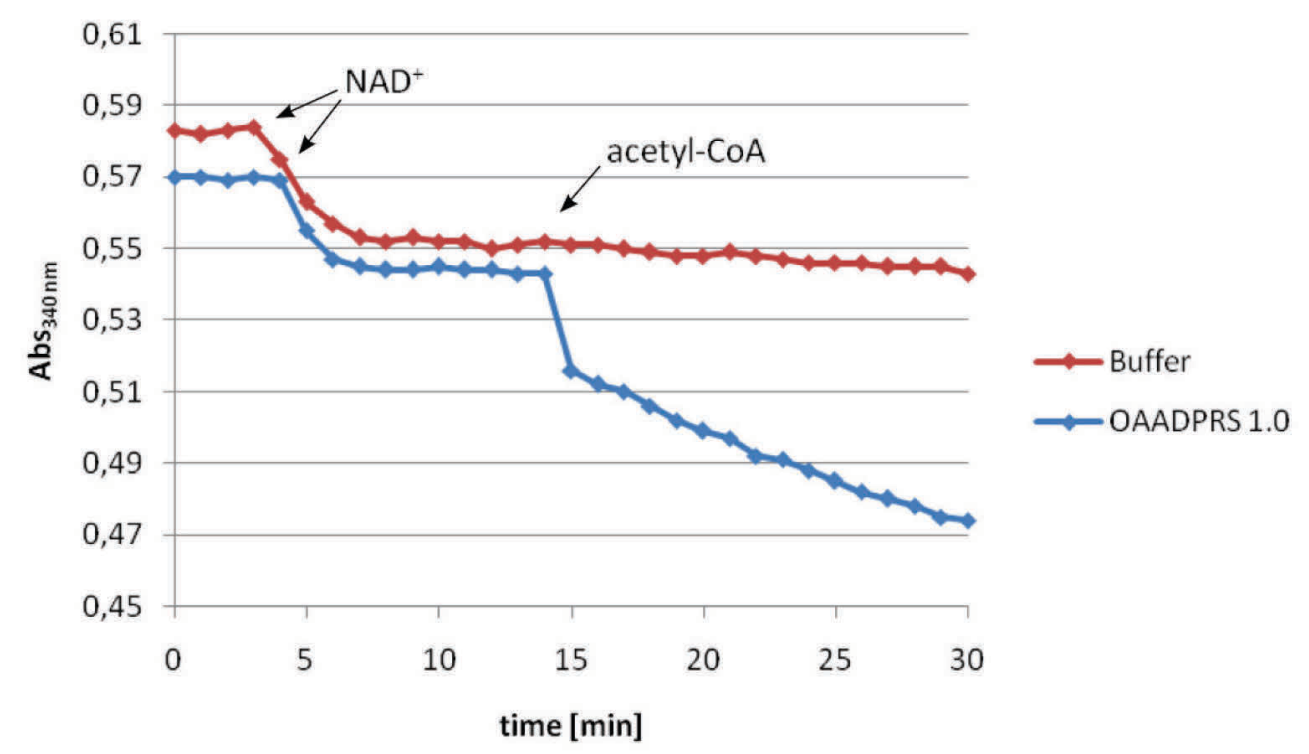

Figure 3.6: Enzymatic coupled sirtuin assay with synthase 1.0. Arrows indicate the time points of $\operatorname{NAD}^{+}$and acetyl-CoA addition. A decrease of absorption after $\mathrm{NAD}^{+}$addition was due to nicotinamide contamination. OAADPRS 1.0 activity was dependent on the addition of acetyl-CoA since a significant decrease of the absorption was only obtained after its addition. The negative control, containing buffer instead of synthase, did not show an absorption decrease upon acetyl-CoA addition. 
These experiments demonstrated the dependence of the activity of synthase 1.0 on acetyl-CoA and thus indicated, that also the acetyltransferase moiety of the synthase was active.

After the activity of synthase 1.0 was confirmed, experiments were performed to optimize its activity. Since the CobB and the Hat1 moieties were connected by a peptide chain, different length of this linker were analyzed to determine if the activity of the synthase could be improved. Therefore, mutations were introduced to the histone $\mathrm{H} 3 / \mathrm{H} 4$-part of the peptide chain by mutagenesis PCR. These mutations were deletions of 5 or 10 amino acids to shorten the linker region. For clarification resulting synthases were named synthase 1.1 and synthase 1.2 (Figure 3.7).

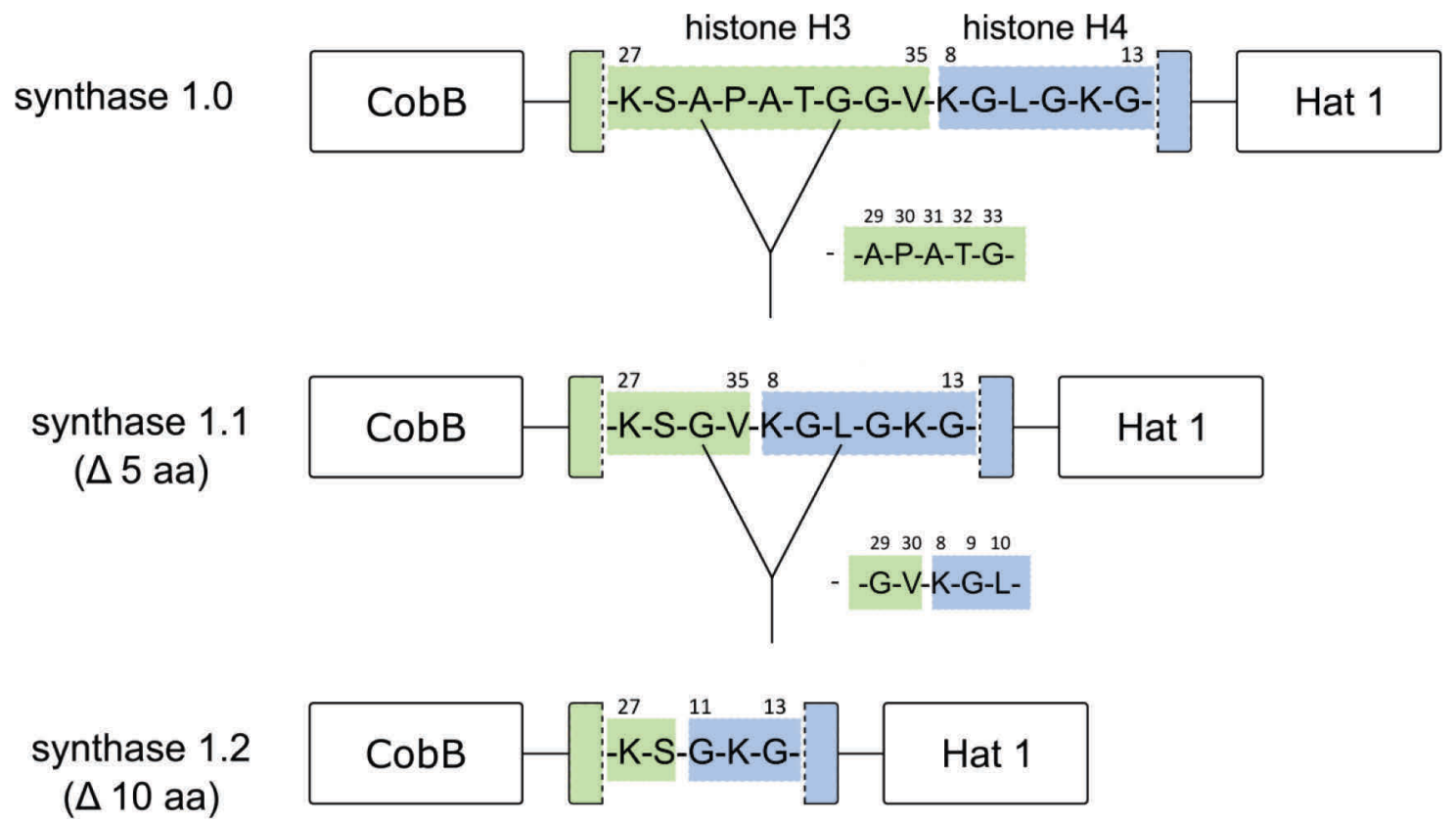

Figure 3.7: Schemes of the three $\boldsymbol{O}$-acetyl-ADP-ribose synthases. Synthase 1.1 was shortened by five amino acids in the histone $\mathrm{H} 3$ part of the linker sequence. In synthase 1.2 ten amino acids of the linker sequence were deleted.

For comparison of the activity of the original synthase 1.0 and the two modified versions 1.1 and 1.2 , the enzymatic coupled sirtuin assay was utilized. Figure 3.8 visualizes the decrease of NADPH absorption in the different samples, representing the activity of the 
synthases, respectively. Absorption of NADPH was measured over ten minutes after the addition of acetyl-CoA. Synthase 1.1 showed the highest decrease in absorption of all three synthases and therefore the highest activity. A deletion of ten amino acids in the linker region of synthase 1.2 led to a decreased activity of the synthase, which was lower than the activity of the original synthase. Turnover numbers were calculated from the slopes and listed in Table 3.1 together with the value of the human deacetylase Sirt1 as a reference. ${ }^{59}$ Synthase 1.1 showed the highest turnover number, which is comparable to free Sirt1 deacetylase. The original synthase 1.0 had a slightly lower turnover number. Synthase 1.2 showed with only $27 \%$ of the value of synthase 1.1 the lowest turnover of all three synthases. In summary, a deletion of five amino acids in the linker region in synthase 1.1 produced the highest increase of activity. Therefore, synthase 1.1 was chosen for further experiments investigating the effects of the metabolite OAADPR.

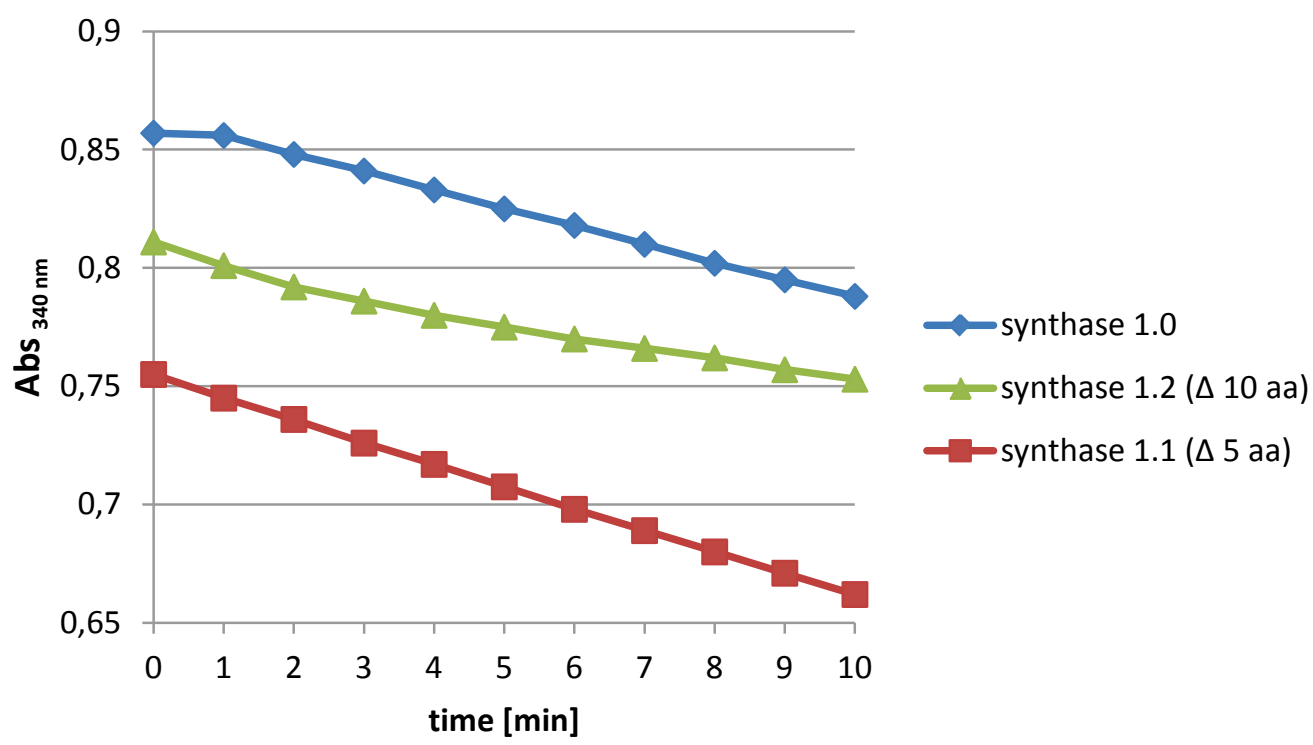

Figure 3.8: Measurement of the activity of the three different synthases with the enzymatic coupled sirtuin assay. The diagram shows the slopes of the decrease of NADPH absorption after addition of acetylCoA to the reaction mixture and hence indicates the activity. Synthase 1.1, with five deleted amino acids in the linker peptide, showed the highest activity of the three synthases. A deletion of ten amino acids in synthase 1.2 led to a reduced activity compared to the two others. 
Table 3.1: Turnover numbers of the three synthases and the human sirtuin Sirt1.

\begin{tabular}{cc}
\hline Deacetylase & $\boldsymbol{k}_{\text {cat }}\left(\mathbf{s}^{-1}\right)$ \\
\hline synthase 1.0 & $0.046 \pm 0.001$ \\
synthase 1.1 & $0.059 \pm 0.008$ \\
synthase 1.2 & $0.016 \pm 0.001$ \\
Sirt1 & $0.060 \pm 0.003$ \\
\hline
\end{tabular}

As a negative control for further experiments an inactive mutant of synthase 1.1 was made by mutagenesis PCR. The aim was to replace the substrate lysine with an amino acid that could not carry an acetyl group. Thus, the main substrate for the deacetylase would not be available and no target for the acetyltransferase. Consequently, the activity of the synthase should be reduced to approximately zero. The mutant carried a glycine in place of the substrate lysine. To confirm the replacement of the substrate lysine in this new mutant and the inability to bind acetyl groups, it was spotted on a membrane together with the active synthase 1.1 and synthase 1.0 as controls (Figure 3.9). The membrane was treated with an acetyllysine antibody and decorated with an ECL reagent. Spots of synthases 1.0 and 1.1 showed a bright signal, whereas the spot of the mutated synthase displayed only a weak signal. This weak signal could possibly be due to another lysine in the enzymatic moieties of the synthase which could be acetylated. The bright signals of synthases 1.0 and 1.1 demonstrated the presence of acetyllysine, which originate from the substrate lysines because the replacement of this amino acid was the only change made in the inactive mutant. To verify that the signals in the synthases 1.0 and 1.1 represented the acetylated substrate lysines, a deacetylation reaction was performed. All three synthases were mixed with $\mathrm{NAD}^{+}$, the co-substrate of the deacetylase moiety, and incubated for $20 \mathrm{~min}$. Afterwards the reaction mixtures were spotted on the membrane and treated as above. No signals for acetylated lysine could be detected, which led to the conclusion that the acetyl groups were cleaved. Summarized, 
these experiments proved the replacement of the substrate lysine by a non-acetylated amino acid in the mutant and therefore its highly decreased activity.
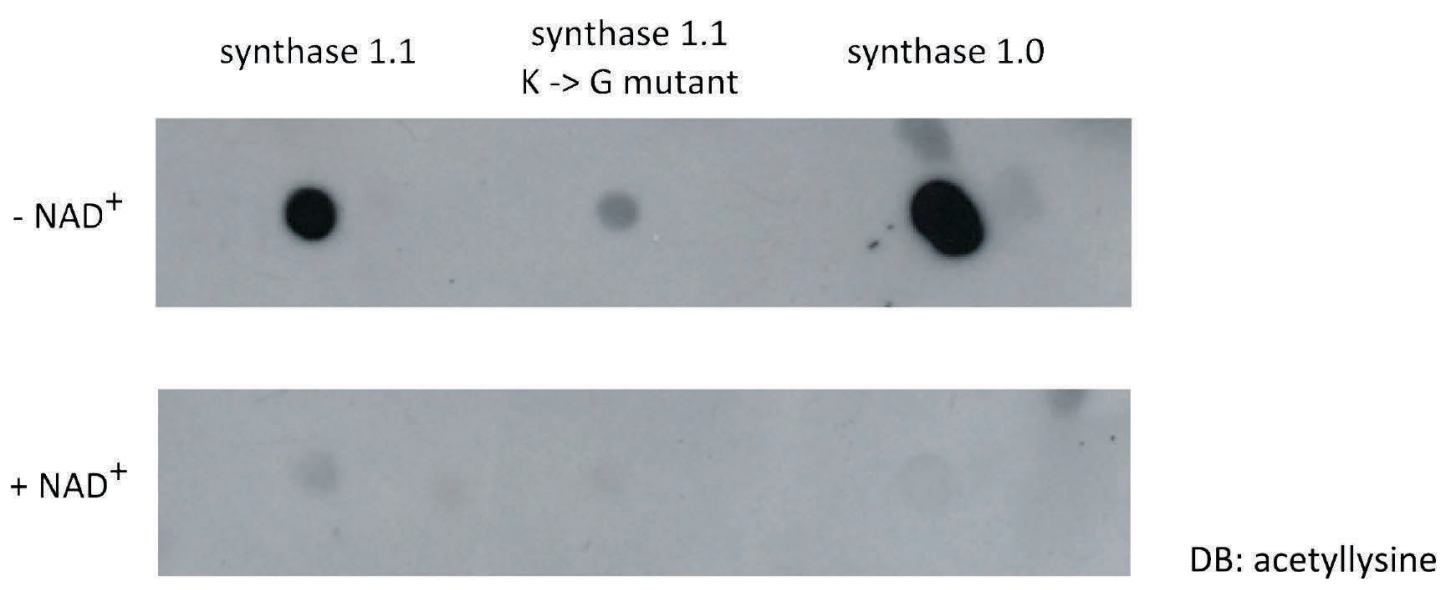

Figure 3.9: Dot Blot of the two active synthases 1.0 and 1.1 and the mutated version of synthase 1.1 where the substrate lysine was replaced by a glycine. The membrane was decorated with an acetyllysine antibody followed by an ECL reagent. In the upper section the samples were spotted untreated and the active synthases showed a strong signal in contrast to the mutant. In lower section the samples were incubated with $\mathrm{NAD}^{+}$for $20 \mathrm{~min}$, a substrate for the deacetylation. Upon this $\mathrm{NAD}^{+}$addition the signals disappeared which demonstrated a deacetylation of the substrate lysines.

\subsubsection{Validation of the formation of $O$-acetyl-ADP-ribose}

After a functional optimization of the synthase was achieved and the activity was shown, proof of the formation of the metabolite OAADPR was still elusive. Therefore, the product solution of the enzymatic coupled assay was measured by liquid chromatography coupled to tandem mass spectrometry. The mass spectrometer was used as a selective detector and signals of single masses were plotted against time in an extracted ion chromatogram (XIC). The product solution was scanned for masses of OAADPR and ADP-ribose. ADPribose is a degradation product of OAADPR and hence always found in OAADPR containing samples. Its retention time was determined in earlier experiments and served as a standard in the measurment searching for the metabolite. 
The chromatogram, a superimposition of two XICs, showed two signals at retention times of $38.15 \mathrm{~min}$ and $40.61 \mathrm{~min}$ (Figure 3.10). The minor signal had the same retention time as the standard ADP-ribose and also the mass of $558 \mathrm{Da}$ coincided. The major signal at 38.15 min was measured with a slightly higher mass of $600 \mathrm{Da}$ and the difference of $42 \mathrm{Da}$ to the other signal corresponded to an additional acetyl group in the molecule. Hence, it was concluded to represent OAADPR. Altogether, these results verified the formation of the metabolite OAADPR during synthase activity and with it the functionality the synthase was engineered for.

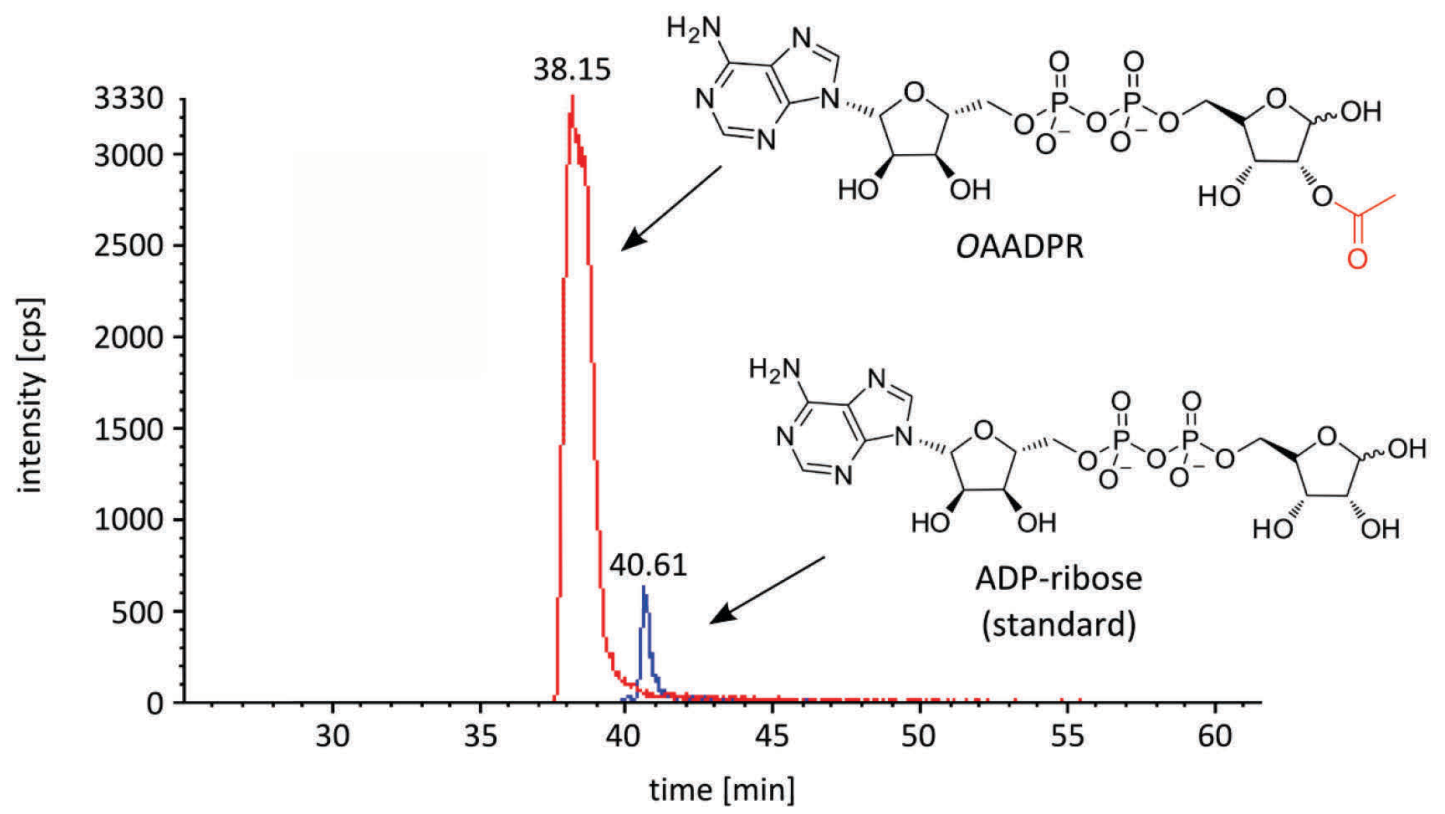

Figure 3.10: A superimposition of two XICs, obtained by mass spectrometry analysis of the product solution of the enzymatic coupled sirtuin assay. The minor signal represents a mass of $558 \mathrm{Da}$ and had the same retention time as the ADP-ribose standard. The major signal at a retention time of $38.15 \mathrm{~min}$ represents a mass of $600 \mathrm{Da}$, which corresponds to the mass of OAADPR.

\subsubsection{Kinetic characterization of the optimized synthase 1.1}

Overall results of the enzymatic sirtuin assay showed that the CobB part of the engineered synthase was able to deacetylate the substrate lysine in the linker region and the acetyltransferase Hat1 was able to acetylate this lysine again. Nevertheless, the effect 
of those two enzymatic moieties being linked remained elusive. The arising question was, if the activity of one enzyme is hampered by the other and how high their activities are in synthase 1.1 compared to uncoupled enzymes. For a kinetic characterization of both enzymatic moieties, the enzymatic coupled sirtuin assay was used. In the first steadystate kinetics acetyl-CoA was titrated from 2 to $64 \mu \mathrm{M}$ into the reaction mixture to analyze the substrate dependent activity of Hat1. $N A D^{+}$, the substrate for CobB, was added in excess to ensure that the substrate lysine was deacetylated at maximum velocity. The acetylation rates obtained in this experiment were plotted against the substrate concentration in a Michaelis-Menten plot (Figure 3.11).

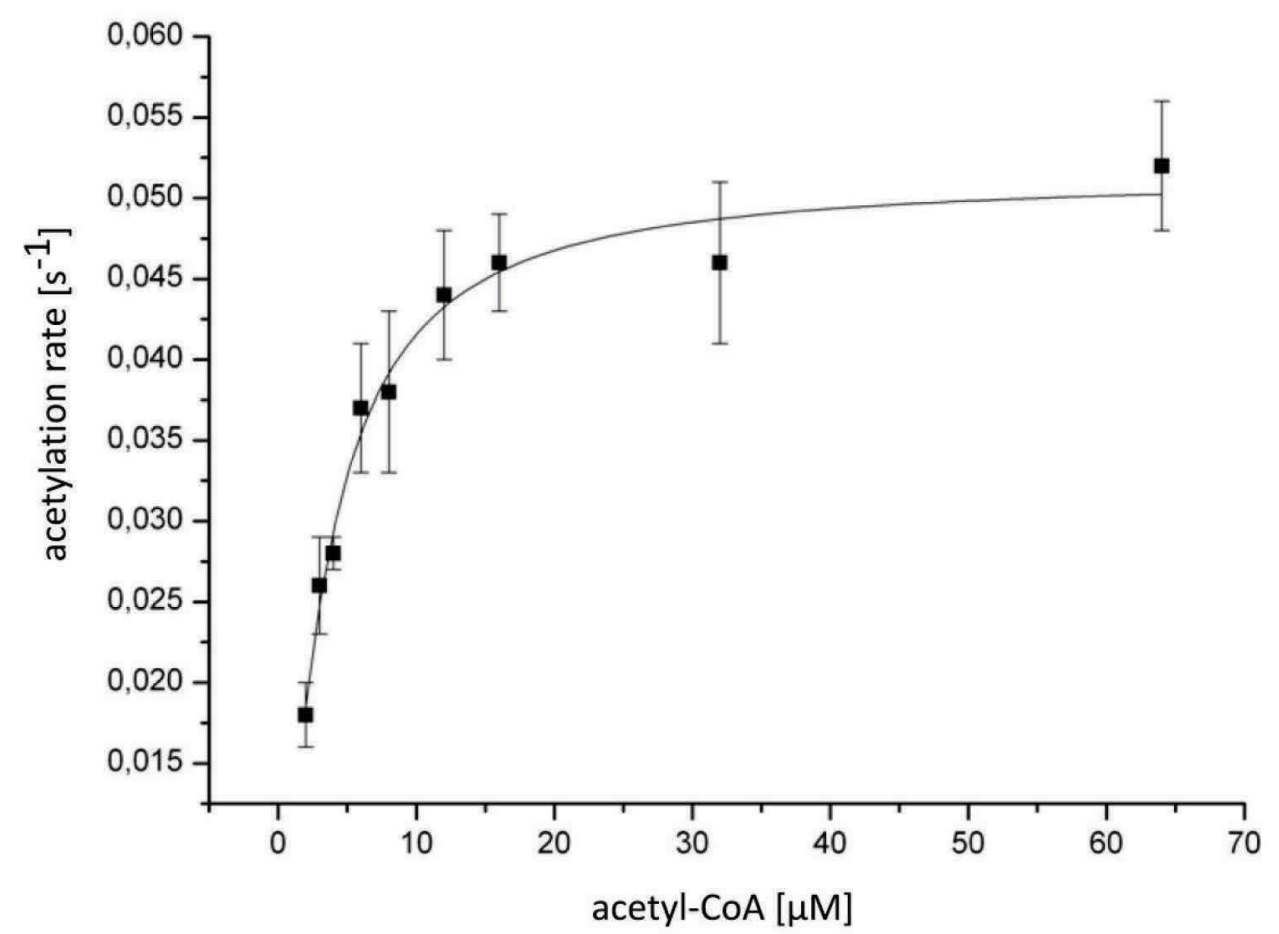

Figure 3.11: Steady-state kinetics of the Hat1 moiety in OAADPRS. Acetylation rates, determined using the enzymatic coupled sirtuin assay, were plotted against the acetyl-CoA concentrations.

The same experiment was performed with the CobB moiety. $\mathrm{NAD}^{+}$was titrated while acetyl-CoA was added in excess. Deacetylation rates were measured and plotted in a Michaelis-Menten plot against the $\mathrm{NAD}^{+}$concentration (Figure 3.12). 


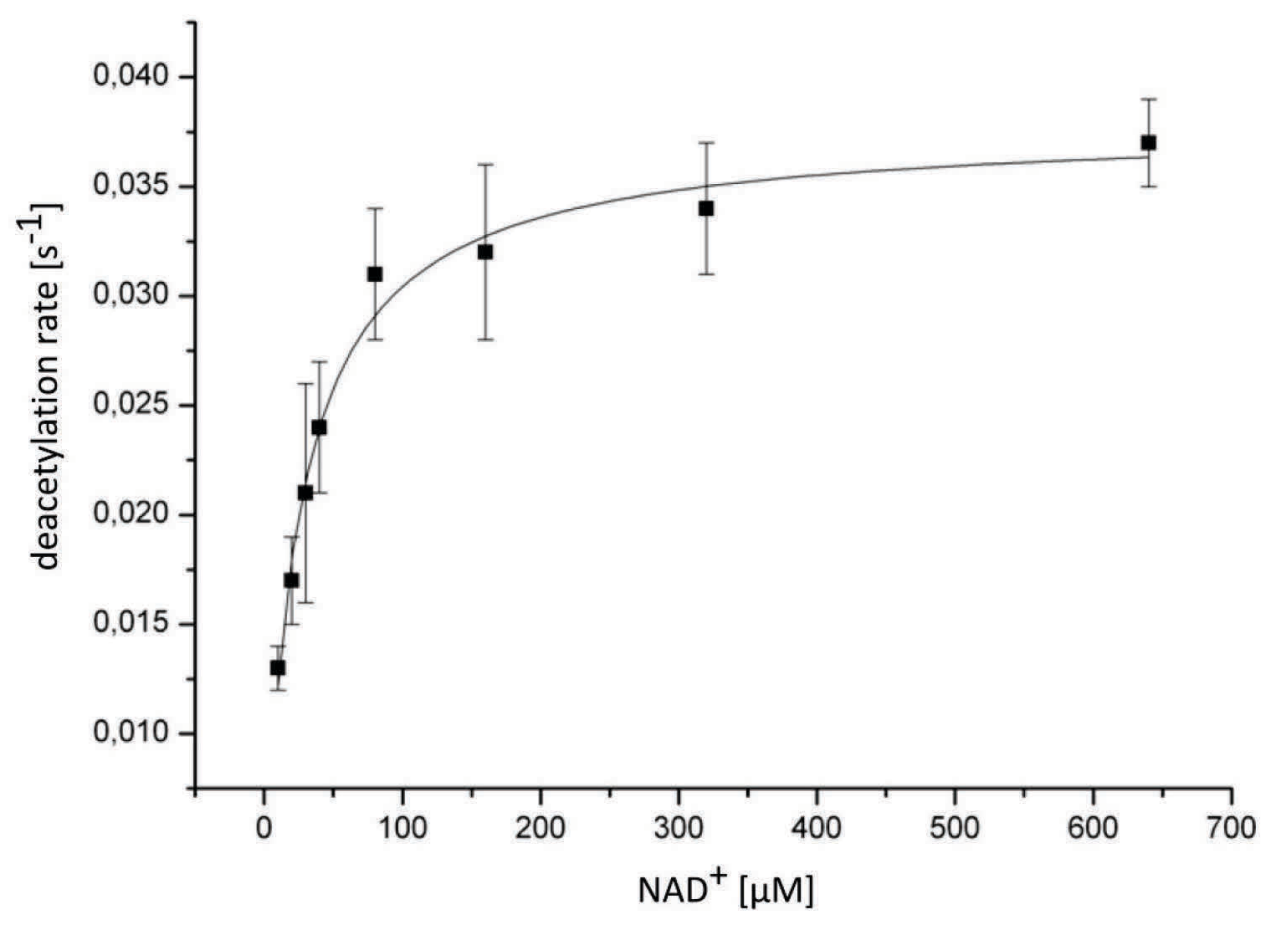

Figure 3.12: Steady-state kinetics of the CobB moiety in OAADPRS. Deacetylation rates, determined using the enzymatic coupled sirtuin assay, were plotted against $\mathrm{NAD}^{+}$concentrations.

Based on the obtained acetylation and deacetylation rates, different kinetic parameters could be calculated. The plots of the acetylation and deacetylation rates against the substrate concentrations were fitted to the Michaelis-Menten equation to determine the values for $v_{\max }, K_{\mathrm{m}}$ and $k_{\text {cat }}$ (Table 3.2 ). The maximum velocity $v_{\max }$ of Hat1 was with $0.8 \mu \mathrm{M} / \mathrm{min}$ about $36 \%$ lower than the maximum velocity of CobB, which had a value of 1.2 $\mu \mathrm{M} / \mathrm{min} . K_{\mathrm{m}}$ is the Michaelis constant and describes the affinity of the enzyme towards its substrate. CobB had a more than 4-fold higher affinity for NAD ${ }^{+}$than Hat1 for acetyl-CoA. The amount of substrate molecules, catalyzed per time unit under substrate saturation, is exhibited in the turnover number $k_{\text {cat }}$. It is calculated from the maximum velocity and concentration of the enzyme. Here, Hat1 had the higher turnover number with $0.06 \mathrm{~s}^{-1}$. The $k_{\text {cat }}$ value for CobB was $0.04 \mathrm{~s}^{-1}$. In summary, the CobB moiety showed a higher maximum velocity and a higher substrate affinity but a lower turnover rate than the Hat1 moiety. The different turnover rates for the two enzymatic moieties were 
unexpected since they share the same substrate. Their velocity depends on how fast the other enzymatic moiety respectively acetylates/deacetylates the substrate.

Table 3.2: Kinetic data of the CobB and Hat1 moieties in synthase 1.1 obtained from the Michaelis Menten equations.

\begin{tabular}{ccc}
\hline & CobB & Hat1 \\
\hline$v_{\max }$ & $1.2 \mu \mathrm{M} / \mathrm{min}$ & $0.8 \mu \mathrm{M} / \mathrm{min}$ \\
$K_{\mathrm{m}}$ & $18.0 \mu \mathrm{M}$ & $4.0 \mu \mathrm{M}$ \\
$k_{\text {cat }}$ & $0.04 \mathrm{~s}^{-1}$ & $0.06 \mathrm{~s}^{-1}$ \\
\hline
\end{tabular}

\subsubsection{Effects of the synthase and the formation of the metabolite OAADPR in Escherichia coli}

It is known that injections of the metabolite OAADPR delay or even block the maturation of starfish oocytes and blastomeres. ${ }^{29}$ Since synthase 1.1 produces this metabolite in the cell, it might lead to a change in phenotype as well. For analysis of the influence of synthase expression and metabolite formation on the phenotype of $E$. coli cells, a growth test was performed. Since synthase 1.1 was the only version used from this point on together with its inactive mutant, for symplification it was called active synthase.

Besides a plasmid with the active synthase, one containing the inactive mutant with the exchanged substrate lysine and an empty plasmid as control were transformed into BL21(DE3) cells. The cell cultures were incubated at $37^{\circ} \mathrm{C}$. Samples were taken every hour and the optical density measured. Growth curves from $40 \mathrm{~min}$ before until four hours after induction are depicted in Figure 3.13. After the expression of the active synthase was induced, a severe delay in growth could be observed. Compared to cells harbouring the inactive mutant and the control, which grew similar to each other, the cells expressing the active synthase reached only half of the optical density in the same time. Dilution 
series on agar plates demonstrated that the cells were severe delayed in growth but still alive.

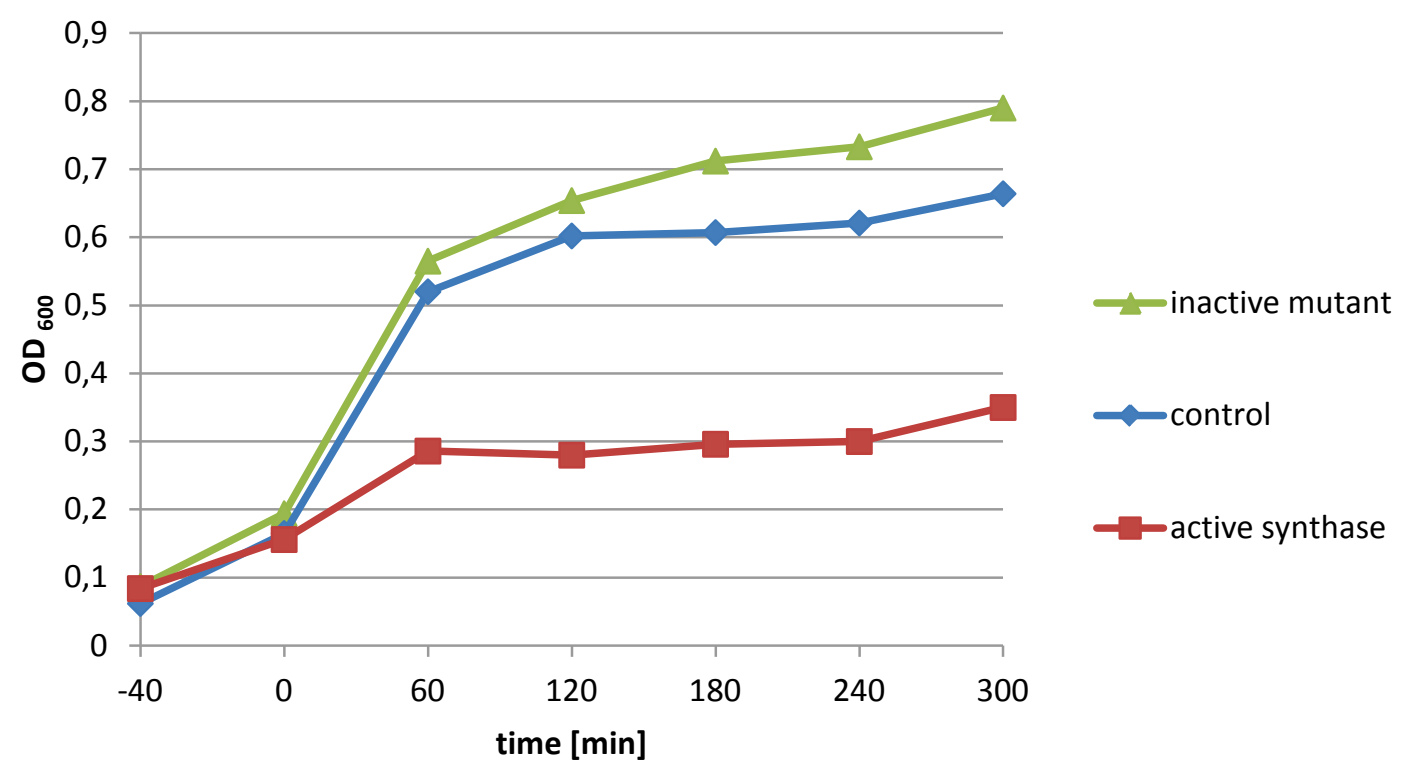

Figure 3.13: Growth test of $E$. coli cells harbouring plasmids with the active synthase or the inactive mutant. The control contained an empty plasmid. Cells were induced with $0.5 \mathrm{mM}$ IPTG at time point zero. Growth of cells expressing the active synthase was severe delayed after induction while cells with the inactive synthase grew comparable to the control.

The growth tests showed that synthase expression and the coupled formation of the metabolite OAADPR had indeed a high influence on cell metabolism.

For further analysis of the changes in the metabolism of the cells harbouring the synthase a tightly controlled expression system was needed. The expression had to be downregulated to a level where the cells did not show such a high growth deficiency. An arabinose inducible promoter, the $\mathrm{P}_{\mathrm{BAD}}$ promoter, was chosen because it can be regulated dose-dependently. The active synthase and the inactive mutant were cloned into a plasmid containing this promoter. Different induction levels from 200 to $10000 \mathrm{ppm}$ arabinose in the cell cultures were tested for synthase expression. Western blot analysis demonstrated that protein was also expressed at the lowest induction level of $200 \mathrm{ppm}$ arabinose (Figure 3.14). Growth tests of cultures with the two induction levels resulted in the same phenotype and no growth delay was detected. 


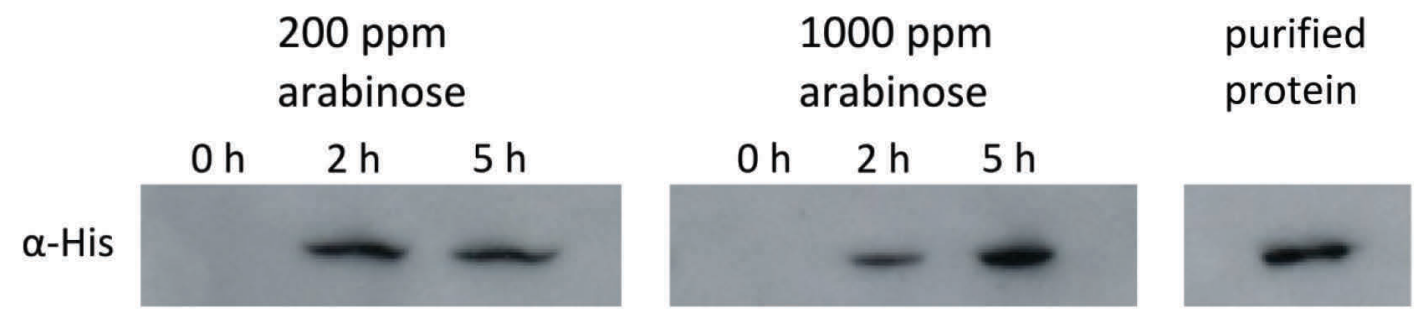

Figure 3.14: Western blot of the active synthase expressed from a plasmid with an arabinose inducible promoter. The amount of synthase expressed, utilizing induction levels of 200 and 1000 ppm arabinose were compared. Protein was detected with an anti-histidine antibody. Samples were taken before, 2 and $5 \mathrm{~h}$ after induction. On the right purified synthase was blotted as control. At both induction levels protein expression was visible after induction.

For analysis of the effects of the synthase expression level and OAADPR formation on cell metabolism, a method was needed to observe metabolic activity. The method used is based on resazurin (7-hydroxy-10-oxido-phenoxazin-10-ium-3-one), a redox dye, which can be reduced to resorufin. Resazurin itself is only weakly fluorescent but resorufin emits a strong flourescence signal at $590 \mathrm{~nm} .{ }^{77}$ The reduction is activated by oxidation of NADH, FADH, FMNH or NADPH. Those coenzymes participate in redox reactions of metabolic pathways and are indicators of the metabolic flux. Hence, the observation of the reduction of resazurin can be used to determine and compare metabolic activity in cell samples.

In two experiments cell cultures expressing the active synthase, its inactive mutant and a negative control were induced with 200 and 1000 ppm arabinose. Samples were taken every hour from the moment of induction untill five hours after. The metabolic activity was determined by using the resazurin-assay (2.2.4.2). Fluorescence signals were normalized to the control samples and are depicted in Figure 3.15. The diagrams detail a dependence of the cell viability on the level of induction. In some samples the fluorescence signal exceeded the value of the control and lay above $100 \%$. This is probably due to an error in cell density measurement. The amount of fluorescent resorufin formed is directly dependent on the number of viable cells and therefore differs with an unequal quantity which leads to values exceeding the control. In Figure $3.15 \mathrm{~A}$ ) 
the fluorescence signals of cells after induction with $200 \mathrm{ppm}$ arabinose are shown. Before induction the signal of the sample with the active synthase was slightly higher than the one with the inactive mutant. This changed after induction. The signal for the active synthase started to decrease after three hours to $70-75 \%$ of the control, whereas the signal for the inactive mutant rose to the level of the control. It remained similar to the control until four hours after induction, where it dropped again to the level of the active synthase. An induction with 1000 ppm arabinose, as shown in Figure 3.15 B), resulted in higher effects on cell viability. Before induction, the sample with the active synthase showed a higher fluorescence signal than the control and the inactive mutant. After the beginning of protein expression this signal decreased. Two hours after induction, it dropped to less than half of the value of the control signal. In the following hours the signal raised again to $61 \%$ of the control. The signal of the cells with the inactive synthase was higher than the control one hour after induction. But it started to decrease to about $80 \%$ of the control value in the last two hours of the experiment.

In summary, the induction with $1000 \mathrm{ppm}$ arabinose led to a higher decrease of the metabolic activity in samples containing the active synthase than an induction with $200 \mathrm{ppm}$ arabinose. This is probably be due to a higher rate of synthase expression and subsequently more metabolite formation. In samples containing the inactive mutant an increase of the induction level had only very little effect on the cell metabolism. 
A) Induction with $200 \mathrm{ppm}$ arabinose

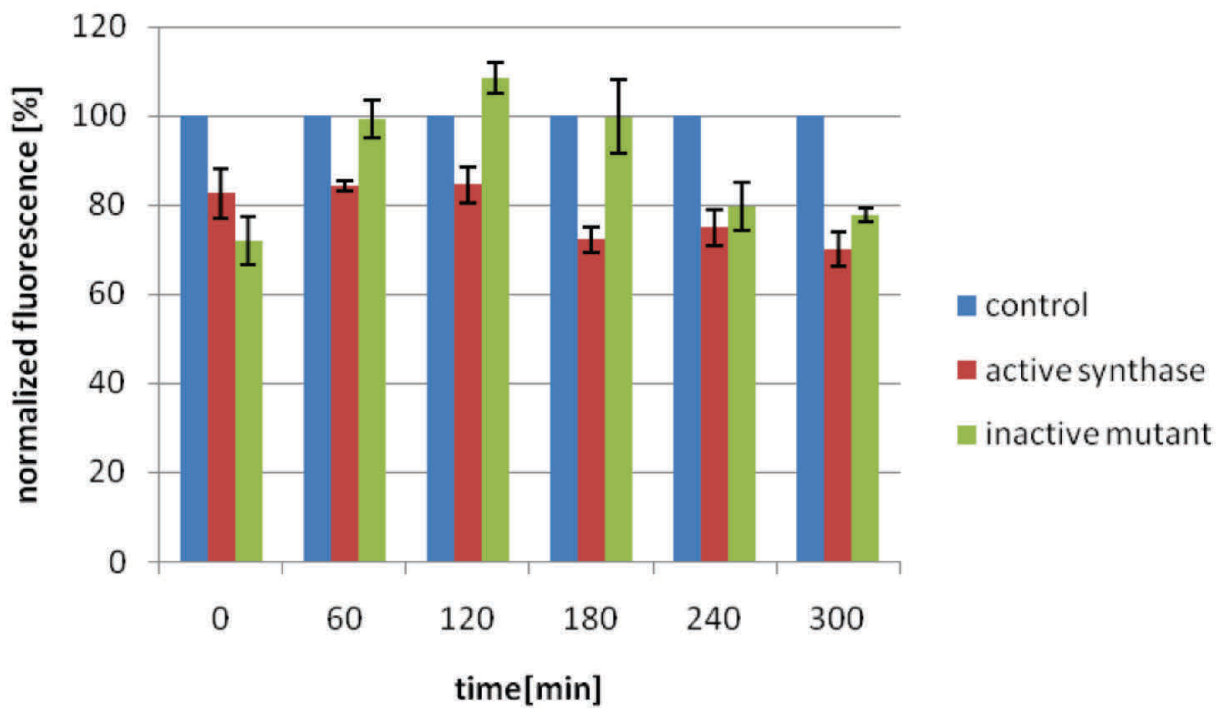

B) Induction with $1000 \mathrm{ppm}$ arabinose

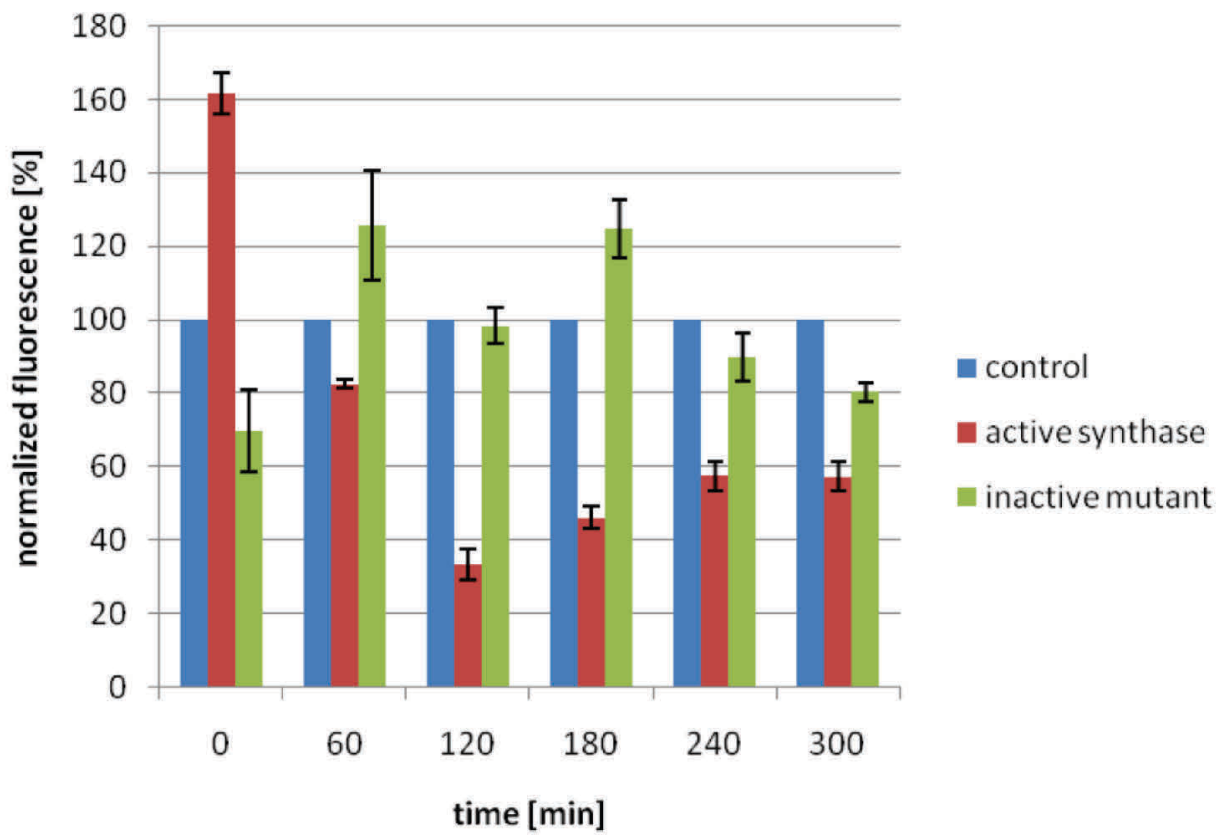

Figure 3.15: Resazurin assay of cells expressing the active synthase, the inactive mutant and a negative control. Samples were taken at indicated time points after induction. The measured fluorescence signals of the formed reagent were normalized to the control, which was set to $100 \%$. In A) cells were induced with 200 ppm arabinose and in B) with 1000 ppm arabinose. A higher expression level of the active synthase and the coupled formation of the metabolite resulted in a higher depression of cell metabolism. 
In unison, the same cell cultures were used to perform another experiment. $\mathrm{NAD}^{+}$levels inside the cells were measured with the EnzyChrom ${ }^{\mathrm{TM}} \mathrm{NAD}^{+} / \mathrm{NADH}$ Assay Kit (2.1.3). Since $\mathrm{NAD}^{+}$is a co-substrate for the synthase, it is likely that the activity of the synthase caused a decrease or maybe even a depletion of the $\mathrm{NAD}^{+}$pool in the cells. This would result in a decrease of metabolic activity depending on the degree of the $\mathrm{NAD}^{+}$reduction and would explain the observed effects. The assay used in this experiment contains a formazan reagent, which is reducable by NADH. NADH is formed in a lactate dehydrogenase cycling reaction depending on the $\mathrm{NAD}^{+}$concentration in the sample (2.2.4.3). The absorption of the formazan reduction product was measured at $565 \mathrm{~nm}$. On the basis of a standard curve, the amount of $\mathrm{NAD}^{+}$in the samples was calculated and plotted in Figure 3.16. At both induction levels, the $\mathrm{NAD}^{+}$concentration of cells expressing the active synthase was significant lower compared to the control. Induction with $200 \mathrm{ppm}$ arabinose resulted in a decrease of $50 \%$ of the $\mathrm{NAD}^{+}$concentration and the induction with $1000 \mathrm{ppm}$ in a $86 \%$ decrease. Samples of the inactive synthase showed a significant smaller reduction of the $\mathrm{NAD}^{+}$pool. After induction with $200 \mathrm{ppm}$ arabinose $15 \%$ less $\mathrm{NAD}^{+}$was detectable than in the control and at the higher induction level $29 \%$ less.

Summarized, the induction of the expression of the active synthase had a highly negative effect on $\mathrm{NAD}^{+}$levels in the cell. A further 5 -fold increase of the induction level from 200 to $1000 \mathrm{ppm}$ arabinose led to a continuing 3,5-fold lower $\mathrm{NAD}^{+}$amount in the cells. Samples containing the inactive mutant showed a much lower effect after induction and no influence by an increase of the induction level.

Since the induction with $200 \mathrm{ppm}$ arabinose led to a synthase expression with a lower impact on the $\mathrm{NAD}^{+}$pool in the cells and a lower decrease of metabolic activity, it was used in subsequent experiments to find possible other effects of the metabolite. 
A) Induction with $200 \mathrm{ppm}$ arabinose

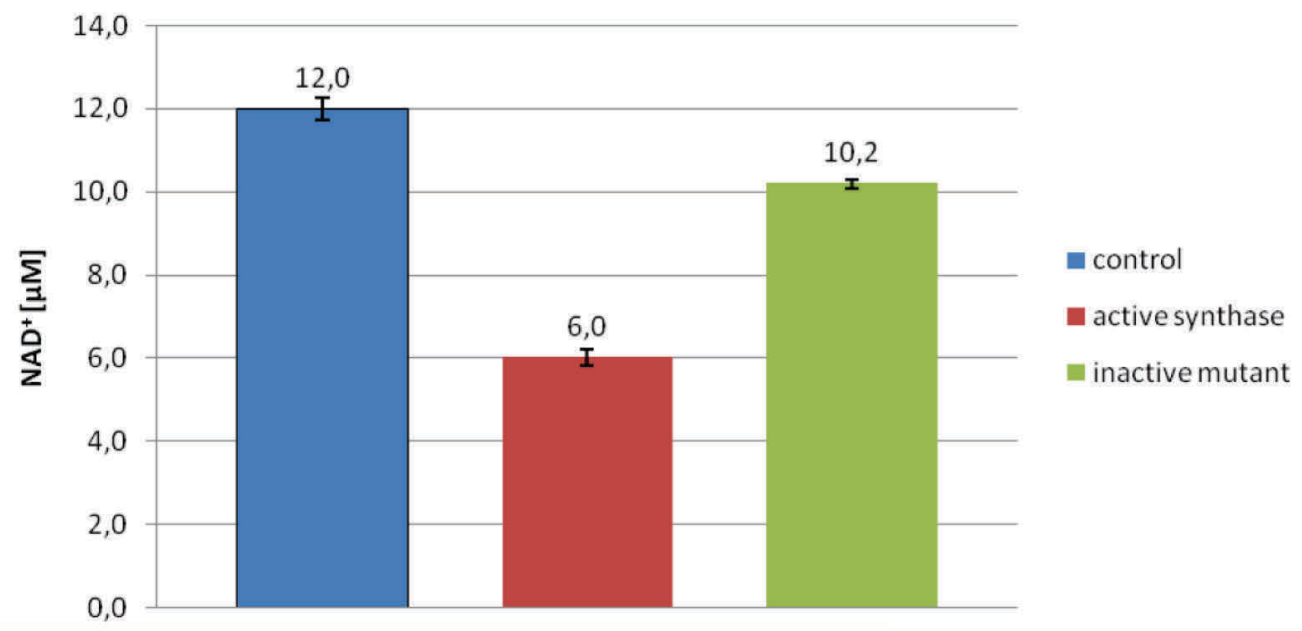

B) Induction with 1000 ppm arabinose

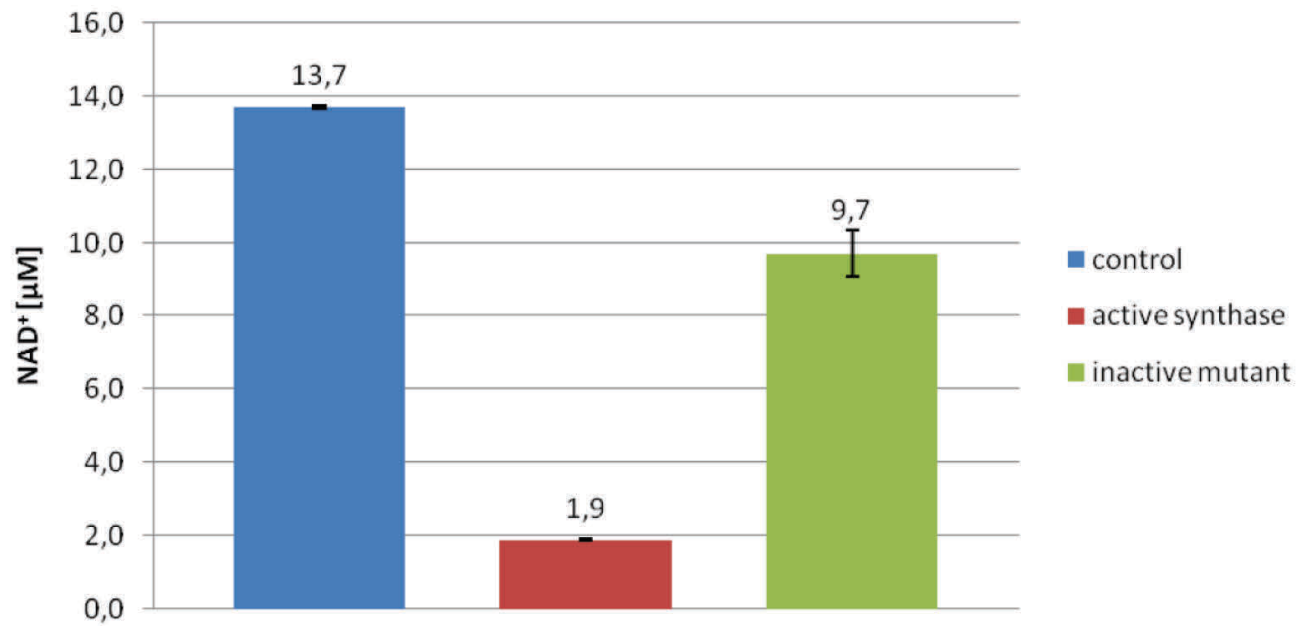

Figure 3.16: Determination of $\mathrm{NAD}^{+}$levels in cells expressing the active synthase, the inactive mutant or a negative control. Samples were taken two hours after induction. A formazan reagent was added and the amount of reduction product, which depends on NADH concentration in the sample, was measured. NAD ${ }^{+}$ amounts were calculated from those NADH concentrations. In A) cells were induced with $200 \mathrm{ppm}$ arabinose and in B) with $1000 \mathrm{ppm}$ arabinose. $\mathrm{NAD}^{+}$levels were severely decreased when the active synthase was expressed and nearly depleted after increasing the induction to $1000 \mathrm{ppm}$ arabinose. Expression of the inactive synthase resulted in a much lower decrease of NAD ${ }^{+}$. 


\subsubsection{Gene expression analysis}

The previous experiments detailed the expression of the synthase and formation of $\mathrm{O}$-acetyl-ADP-ribose and their influence on cell metabolism and $\mathrm{NAD}^{+}$levels in the cell. To obtain further insights into these metabolic changes, a gene expression analysis was performed. This method is used to determine expression levels of genes under defined conditions, and therefore gives information about affected pathways in the cell.

For this experiment, cell cultures with the active synthase, its inactive mutant and the control plasmid were utilized. Protein expression was induced with $200 \mathrm{ppm}$ arabinose and the cells were harvested two hours after. Samples were normalized after cell count followed by extraction of the total RNA using TRI reagent (2.2.1.9). The RNA was electrophoretically separated via a denaturing agarose gel for qualitative and quantitative analysis. Finally, the RNA samples were given to the "Transcriptome Analysis Laboratory (TAL)" at the DNA Microarray Facility in Göttingen, where they were analyzed. First, the samples were transcribed into fluorescent labled cDNA. Subsequently, the cDNA was incubated with probes consisting of oligonucleotide sequences from $E$. coli genes immobilized on a microchip. Fluorescence signals of bound CDNA was measured and assigned to the corresponding genes. From the intensity of the fluorescence signals the regulation of the genes could be determined. A 2-fold or higher regulation of a gene was considered significant.

Three separate surveys were performed. An overview of the numbers of regulated genes is given in Figure 3.17. In the first survey the results from the gene expression of samples with the active synthase was compared to that of the negative control. When the active synthase was expressed and OAADPR formed, 204 regulated genes could be found which were not regulated in the sample with the negative control. When the inactive mutant was assayed against the negative control, 55 less regulated genes were detected. In a final comparison, samples with the active synthase were assayed against the ones with the inactive mutant. The catalytic activity of the synthase led to a regulation of 74 genes. The most eminent changes in gene expression were in the results of this last survey. They showed the influence of the active synthase and therefore the metabolite on gene 
expression and hence on affected pathways. Two of the regulated genes were also listed in the survey of the inactive mutant versus the negative control. They were regulated the same fold and were considered as background. The remaining 72 genes were partially only regulated in this evaluation and partially also in the evaluation of the active synthase versus the negative control. Altogether, 34 of the genes in this survey were upregulated and 40 genes were downregulated.

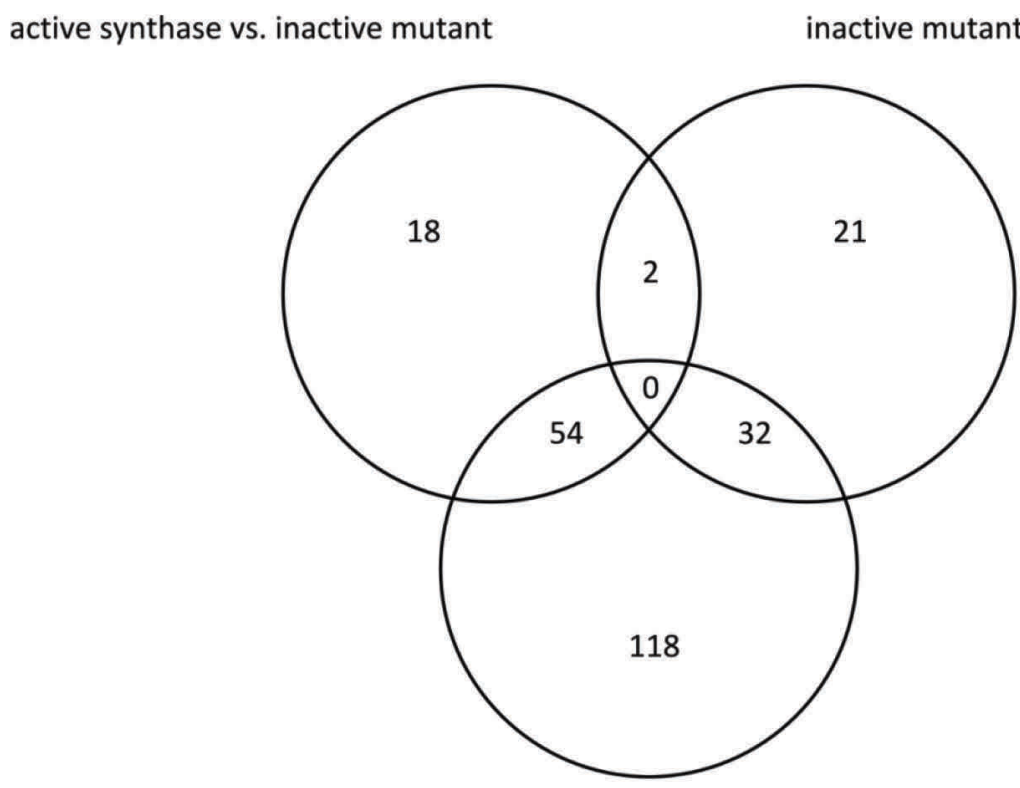

active synthase vs. negative control

Figure 3.17: Venn analysis diagram of the gene expression surveys that were performed. Comparing the active synthase to the negative control, 204 genes were regulated. In the samples of the inactive mutant compared to the negativ control 55 genes were regulated. When gene expression was measured in the presence of the active synthase versus the inactive mutant, 74 genes were regulated. None of the regulated genes were found in all three surveys, only partially in two of them.

Table 3.3 highlights a selection of the 34 upregulated genes, which were more than 2 -fold upregulated (all changes in gene regulation can be obtained in the supplemental information (7.3.1)). Genes were arranged in groups, depending on the pathways they belong to. An exception is the group containing pnuC. The pnuC encoding protein is part of $\mathrm{NAD}^{+}$salvage pathways, while the other genes in the group belong to the de novo $\mathrm{NAD}^{+}$ 
biosynthesis pathway. Thus, they all are connected to $\mathrm{NAD}^{+}$biosynthesis, but through different pathways.

Table 3.3: List of genes with more than 2-fold upregulation in samples with the active synthase versus samples with the inactive mutant. The genes which belong to the same pathway were allocated to the same group.

\begin{tabular}{|c|c|c|}
\hline Symbol & Gene name & $\begin{array}{l}\mathrm{n} \text {-fold of gene } \\
\text { upregulation }\end{array}$ \\
\hline \multicolumn{3}{|c|}{$\mathrm{NAD}^{+}$biosynthesis } \\
\hline ECs0778 & quinolinate synthetase A protein & 14.85 \\
\hline $\operatorname{nad} A$ & quinolinate synthetase & 14.08 \\
\hline $\operatorname{nad} B$ & quinolinate synthase, L-aspartate oxidase (B protein) subunit & 10.18 \\
\hline pnuC & predicted nicotinamide mononucleotide transporter & 4.33 \\
\hline \multicolumn{3}{|c|}{ D-glucarate degradation pathway } \\
\hline garl & alpha-dehydro-beta-deoxy-D-glucarate aldolase & 3.94 \\
\hline garR & tartronate semialdehyde reductase & 3.79 \\
\hline gark & glycerate kinase I & 2.40 \\
\hline \multicolumn{3}{|c|}{$\underline{\text { L-arabinose degradation pathway }}$} \\
\hline $\operatorname{araD}$ & L-ribulose-5-phosphate 4-epimerase & 3.27 \\
\hline $\operatorname{araA}$ & L-arabinose isomerase & 2.71 \\
\hline $\operatorname{araB}$ & L-ribulokinase & 2.24 \\
\hline \multicolumn{3}{|c|}{ phosphoenolpyruvate-carbohydrate phosphotransferase system } \\
\hline fruB & fused fructose-specific PTS enzymes: IIA component/HPr & 2.40 \\
\hline fruK & fructose-1-phosphate kinase & 2.33 \\
\hline uhpt & hexose phosphate transporter & 16.68 \\
\hline yjiY & predicted inner membrane protein & 5.22 \\
\hline soxs & DNA-binding transcriptional dual regulator & 2.47 \\
\hline phoH & $\begin{array}{l}\text { conserved protein with nucleoside triphosphate hydrolase } \\
\text { domain }\end{array}$ & 2.15 \\
\hline
\end{tabular}


In summary, at least four different metabolic systems and pathways were affected when the metabolite was formed in the cells. The highest upregulated genes except of uhpT belong to $\mathrm{NAD}^{+}$biosynthesis. This is probably due to the detected lower $\mathrm{NAD}^{+}$level in cells where the metabolite is formed.

In the same survey 40 genes were downregulated when the synthase was active. A selection is depicted in Table 3.4. Some of them obtain similar functions or are connected by usage of the same substrates. These genes are allocated in groups like ompA, Ecs3221 and Ecs1333. All three genes encode membrane proteins.

Table 3.4: Genes with more than 2-fold downregulated transcription in samples harbouring the active synthase compared to samples with the inactive mutant. Genes belonging to the same pathway or having connected functions were clustered.

\begin{tabular}{llc}
\hline Symbol & Gene name & $\begin{array}{c}\text { n-fold of gene } \\
\text { downregulation }\end{array}$ \\
\hline cell membrane & 3.93 \\
ompA $\quad$ outer membrane protein & 2.03 \\
Ecs3221 $\quad$ outer membrane protein & 2.58 \\
Ecs1333 putative membrane protein & \\
PRPP biosynthesis l, ATP synthesis/hydrolysis & \\
prs & ribose-phosphate diphosphokinase & 3.05 \\
papD & subunit of ATPsynthase F ${ }^{0}$ complex & 2.82 \\
glyS & glycine tRNA synthetase subunit & 2.25 \\
stress response & \\
yifE & conserved protein & 2.96 \\
yjiD & synonym of iraD, an inhibitor of sigma factor $\sigma^{5}$ proteolysis \\
tricarboxylic acid cycle & 2.36 \\
aceA & isocitrate lyase & \\
aceB & malate synthase A & 2.34 \\
& & 2.30 \\
Ecs2845 & glycosyl transferase \\
gnd & gluconate-6-phosphate dehydrogenase & \\
\hline
\end{tabular}




\begin{tabular}{llc} 
Symbol & Gene name & $\begin{array}{c}\text { n-fold of gene } \\
\text { downregulation }\end{array}$ \\
\hline gpmA & phosphoglyceromutase 1 & 2.71 \\
nupC & nucleoside transporter & 2.49 \\
typA & member of ribosome-binding GTPase superfamily & 2.47 \\
hmp & nitric oxide dioxygenase & 2.34 \\
glgP & glycogen phosphorylase & 2.27 \\
lysA & diaminopimelate decarboxylase & 2.16 \\
speA & arginine decarboxylase & 2.08 \\
\hline
\end{tabular}

Here, four groups of downregulated genes could be found with connected functions or pathways but almost as many genes belong to other pathways.

In general, the formation of the metabolite caused the regulation of a variety of genes. Affections of certain pathways by upregulated genes could be expected after the results of the analyses before.

\subsubsection{Colorimetric ß-galactosidase assay}

In the gene expression analysis the hexose phosphate transporter gene uhpT showed the highest transcription with an over 16-fold upregulation, when the active synthase was expressed compared to the inactive mutant. To confirm this result, a promoter reporter ß-galactosidase assay was performed.

This assay is based on the formation of o-nitro-phenol (ONP) from o-nitrophenyl-ß-Dgalactopyranosid (ONPG) by ß-galactosidase (Figure 3.18). ONP can be quantified fluorometrically by measuring the absorption at $420 \mathrm{~nm}$. 


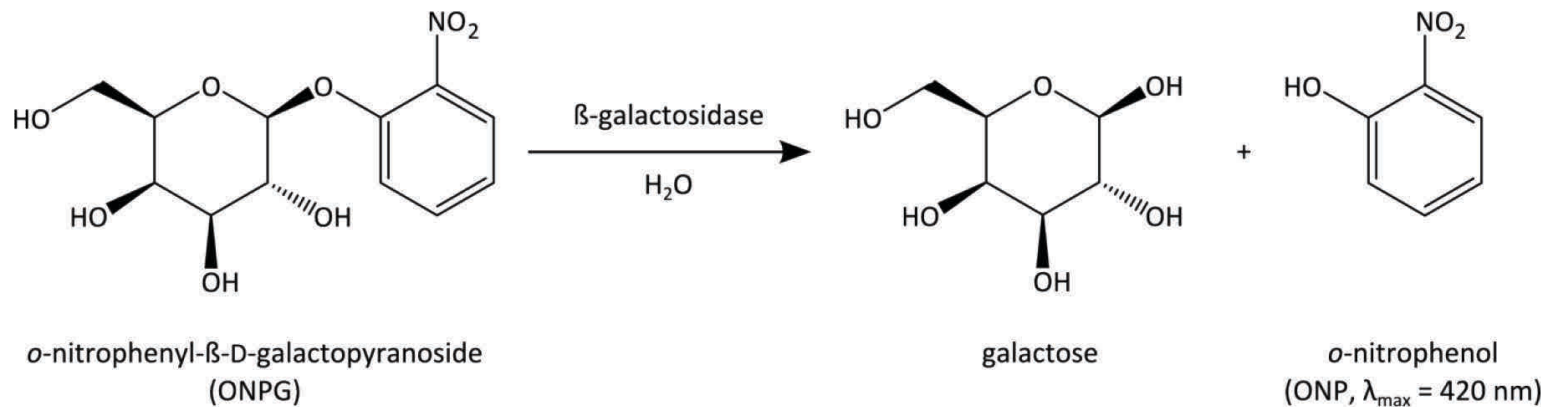

Figure 3.18: Reaction scheme of the ß-galactosidase assay. $o$-nitrophenyl- $ß$-D-galactopyranoside (ONPG) is enzymatically converted to galactose and $o$-nitrophenol (ONP) by the lacZ encoded B-galactosidase. ONP formation can be quantified by measuring its absorption at $420 \mathrm{~nm}$.

For this assay, a plasmid harbouring the DNA coding sequence for ß-galactosidase was modified. The native promoter was replaced by the promoter region of the uhpT gene (Figure 3.19). This plasmid was transformed into E. coli cells together with a plasmid either containing the active synthase, the inactive mutant or the negative control.

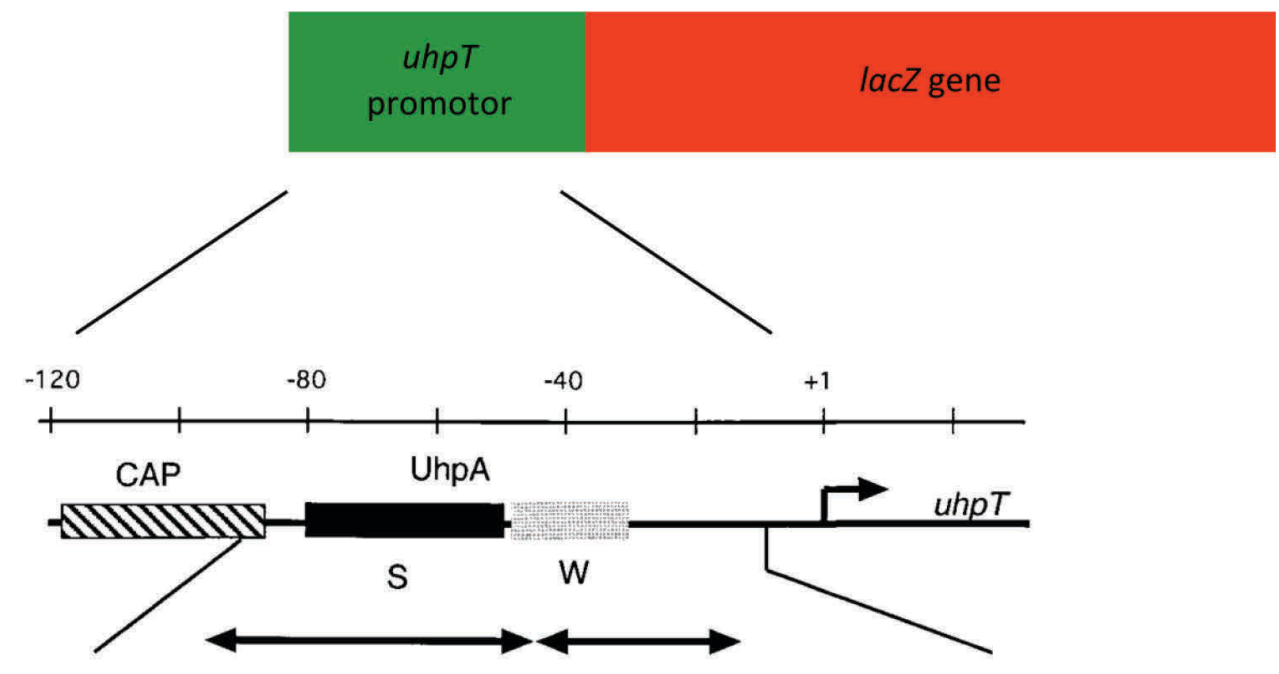

Figure 3.19: Scheme representing the lacZ gene encoding for B-galactosidase and the new promoter region. ${ }^{\text {modified from } 78}$ The original promoter was replaced by the $u$ hpT promoter sequence, including a CAP binding site, a strong-affinity and a low-affinity UhpA-binding region. Depending on synthase and metabolite presence, the promoter should be activated and induce lac $Z$ gene transcription and subsequently ß-galactosidase expression. 
The cell cultures were grown under similar conditions as for the gene expression analysis. In cells expressing the active synthase and forming the metabolite the uhpT promoter was expected to be induced at the same level as in the gene expression analysis. Consequently, also the expression of the ß-galactosidase would be at this level. A particular amount of ONPG would be converted into ONP, which could be subsequently quantified.

For the analysis, cell samples were taken at different time points after the induction with 200 ppm arabinose. The cells were permeabilized and mixed with ONPG. After a certain period of time, when the samples turned yellow due to the formation of ONP, the reaction was stopped by addition of sodium carbonate. The cells were pelleted and the clear supernatant was transferred into a microplate and the absorption measured at $420 \mathrm{~nm}$. Miller units were calculated using Formula 3.1. $\Delta t$ indicates the period of time from addition of the ONPG untill the end of the reaction. $V$ displays the sample volume. The resulting Miller Units were blank corrected and normalized to samples with the control plasmid.

$$
1 \text { Miller Unit }=1000 \times \frac{A b s_{420}}{\Delta t \times V \times A b s_{600}}
$$

Figure 3.20 details the percentages of the measured expression levels of ß-galactosidase relative to the control, which was set to $100 \%$. Measurements were performed one, two and three hours after induction. All samples containing the active synthase and the inactive mutant exhibited a lower expression level of ß-galactosidase than the control. The values for the active synthase decreased from $77 \%$ one hour after induction to $63 \%$ two hours later. Amounts of ß-galactosidase in samples with the inactive mutant decreased from initially $68 \%$ to $38 \%$. Taking the error into account, the values for the active synthase and the inactive mutant were similar in the first hour after induction. Afterwards the values started to diverge. The ß-galactosidase expression in the samples with the inactive mutant decreased significantly while it only slightly deecreased in the samples with the active synthase. In general, the biggest change in the ß-galactosidase 
expression was observed from the first to the second hour after induction. The samples of the active synthase even showed a slight increase in ß-galactosidase expression from the second to the third hour after induction.

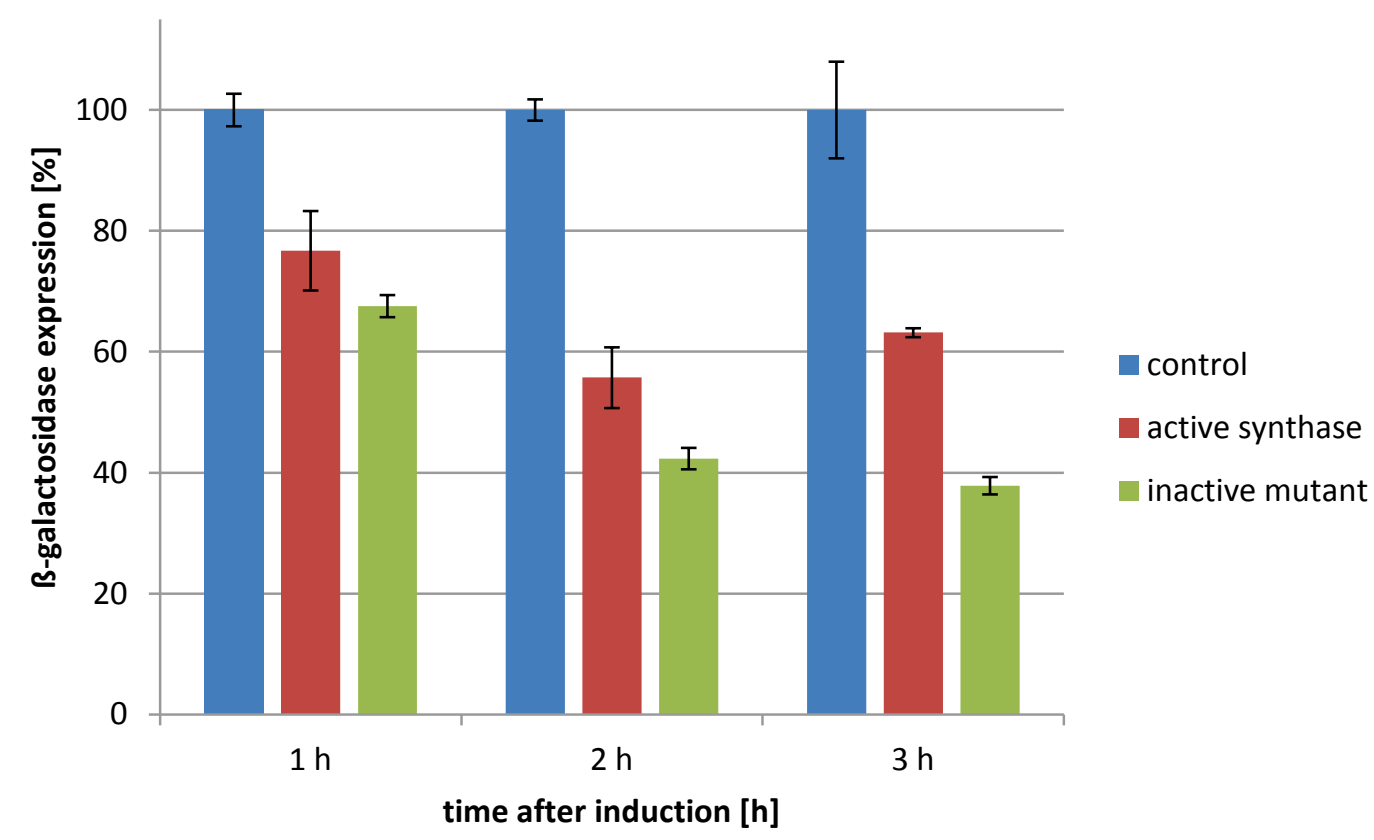

Figure 3.20: Levels of expressed ß-galactosidase one, two and three hours after induction. All values are depicted in percentage and were normalized to the control, which was set to $100 \%$. Samples harbouring the inactive mutant showed a lower ß-galactosidase expression than the samples with the active synthase.

Overall, the observed little difference in expression of ß-galactosidase in samples harbouring the active synthase and the inactive mutant was unexpected and did not match the results from the gene expression analysis. There, a 16-fold difference in gene expression was observed. Thus, the results of the colorimetric ß-galactosidase assay could not verify if the expression of the gene uhpT was upregulated.

\subsubsection{Transcription analysis via real-time PCR}

As already stated above, the microarray gene expression analysis and the ß-galactosidase assay did not show concordant results concerning the gene uhpT. Therefore it was asked, if the gene upregulation could be confirmed, although no changes on the translational 
level were detectable. In order to determine this, quantitative real-time PCR was used, a method for analysing transcription levels of specific genes. For quantification of the gene transcripts, a fluorophore was added to the PCR mixture which emited a signal through binding to newly synthesized dsDNA. The signal intensity increased with each amplification round correlating to product accumulation and was measured at $521 \mathrm{~nm}$.

For this experiment RNA was isolated from E. coli cell samples and cDNA synthesized using a kit (2.1.3). A standard PCR was performed with the cDNA to confirm that the target DNA could be amplified. For unknown reasons the uhpT gene sequence could not be amplified from cDNA but only from genomic $E$. coli DNA. Hence, the experiments were performed with other upregulated genes from the list of the gene expression analysis (Table 3.3) to analyze if these results correlate with each other.

The experiments were performed with cell cultures expressing the active synthase or its inactive mutant. RNA was isolated from cell culture samples (2.2.1.9) and transcribed into cDNA (2.2.1.10). cDNA sequences belonging to the genes of interest were quantified in real-time PCR using appropriate primer pairs. Values of the crossing point $(\mathrm{CP})$ are listed in Table 3.5. The CP is defined as the threshold, where the fluorescence signal raises above the background noise. The lower the value of this crossing point, the more cDNA was initially existing in the sample. From the four genes that were analyzed, three samples reached a lower $\mathrm{CP}$ value when the active synthase was present. The $\mathrm{CP}$ values were higher when the inactive mutant was present in the samples. Only pnuC showed the opposite behaviour. 
Table 3.5: CP values of genes measured by real-time PCR. The left column showes CP values obtained from samples containing the active synthase. In the right column values from samples in which the inactive mutant was expressed are depicted.

\begin{tabular}{lll}
\hline Gene name & $\begin{array}{l}\text { CP value } \\
\text { (active synthase expressed) }\end{array}$ & $\begin{array}{l}\text { CP value } \\
\text { (inactive mutant expressed) }\end{array}$ \\
\hline nadA & 29,85 & 30,16 \\
nadB & 28,21 & 28,94 \\
pnuC & 29,77 & 28,76 \\
fruB & 27,89 & 28,70 \\
\hline
\end{tabular}

From the $C P$ values of the genes of interest and the reference gene gapA, the transcription ratio between samples with the active synthase and the inactive mutant could be determined. gapA is a housekeeping gene whose transcription is not regulated. Its transcription level is considered as the standard transcription level in the cell. Therefore, the value was subtracted from the transcription level of the genes of interest to reveal a potential regulation. These ratios are depicted in Figure 3.21. Only for the gene $n a d B$ a significant higher transcription level could be determined in samples with the active synthase compared to samples with the inactive mutant. As already indicated by the values in Table 3.5, pnuC gene transcription was only increased when the inactive mutant was present and not the active synthase.

In general, it has to be considered that these values are preliminary results since more measurements are needed to obtain adequate error values for all samples. The present error values are calculated from two measurements and their range is in some cases too broad to determine if transcription ratios differ from each other. Additionally, the efficiency of the primers, which was not obtained to that time point, might influence the results.

Summarized, these preliminary values do not reflect the gene regulation values obtained from the gene expression analysis. There, significant regulations have been measured when the active synthase was expressed and the metabolite formed compared to the inactive mutant. 


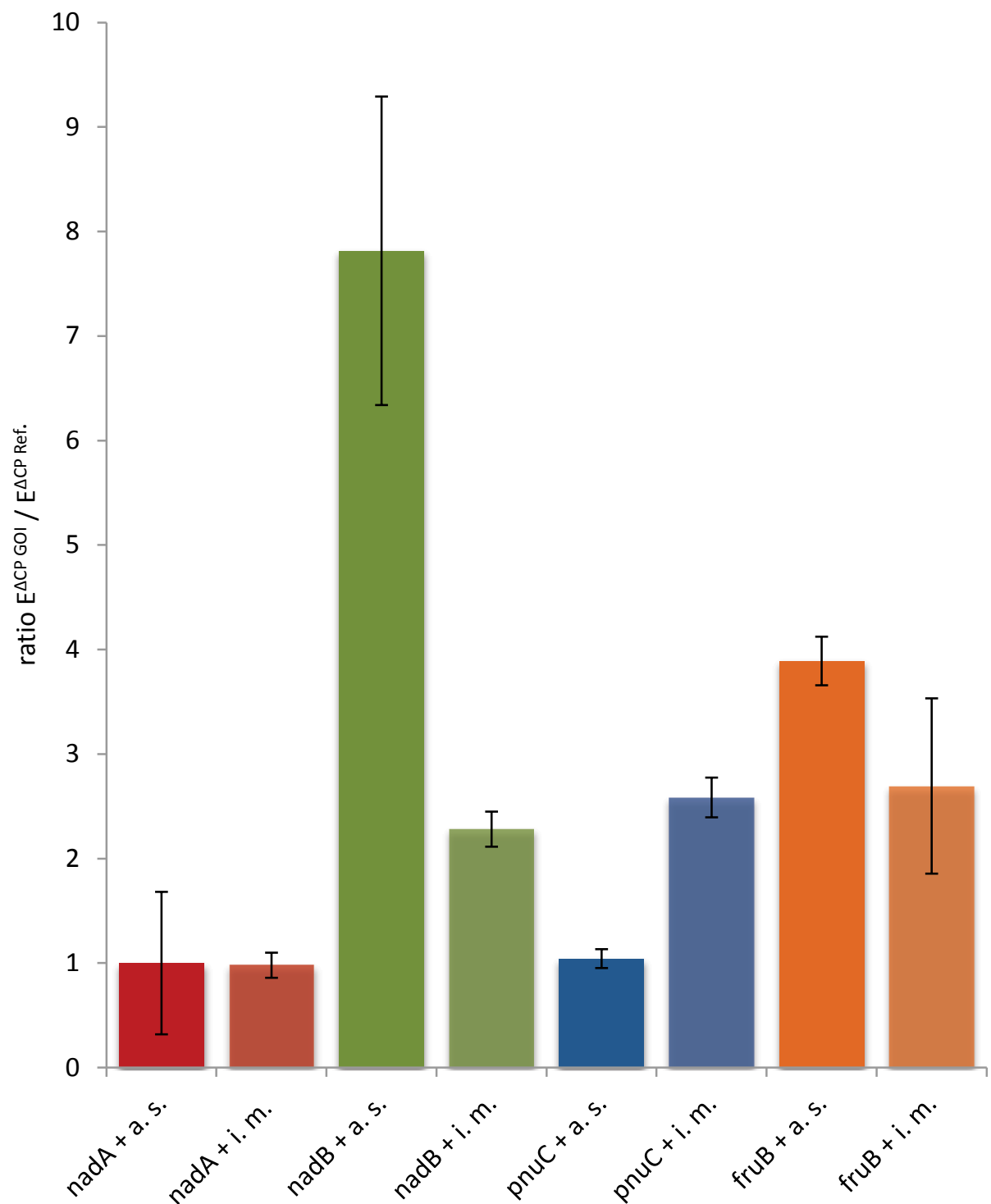

Figure 3.21: Comparison of transcription ratios of genes from Table 3.5, transcribed in the presence of the active synthase (a. s.) or the inactive mutant (i. m.). As reference the housekeeping gene gapA was used and its transcription level was substracted from the values of the genes of interest. nadB was the only gene where, depending on the presence of the active synthase, a significant difference in the transcription level could be determined. The transcription of the pnuC gene was increased in the presence of the inactive mutant. 


\subsubsection{Chemical synthesis of the metabolite $O$-acetyl-ADP-ribose}

The first total synthesis of the metabolite was published by Comstock and Denu. ${ }^{79}$ It was a twelve-step reaction, yielding a mixture of 2'-O-acetyl-ADP-ribose and 3'-O-acetyl-ADPribose. In 2011, a non enzymatic one-step synthesis of OAADPR from NAD ${ }^{+}$was published (Figure 3.22). ${ }^{80}$ This protocol (2.2.5) was followed to prepare the metabolite for later use in in vitro assays to study interactions in gene silencing.
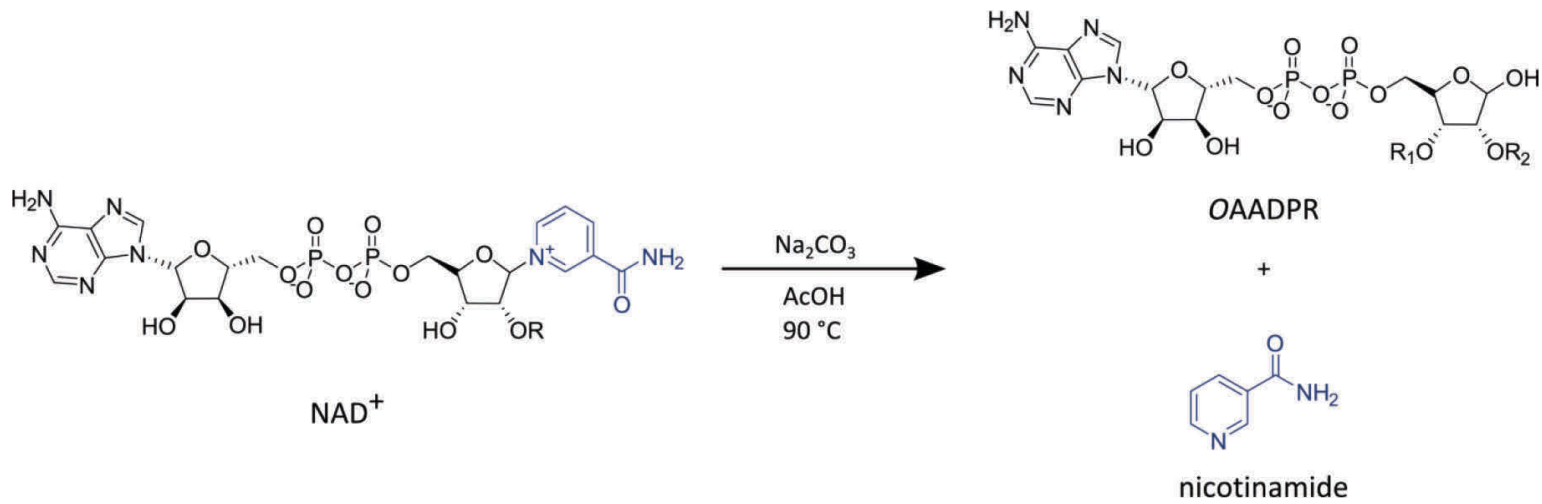

Figure 3.22: Reaction scheme for a one-step synthesis of $O A A D P R .{ }^{\text {modified from } 80}{ }^{8 A D^{+}}$is mixed with sodium carbonate and glacial acetic acid, followed by heating to $90^{\circ} \mathrm{C}$. The product solution containes besides nicotinamide an isomeric mixture of 2'-OAADPR $\left(\mathrm{R}_{1}=\mathrm{Ac}, \mathrm{R}_{2}=\mathrm{OH}\right)$ and 3'- OAADPR $\left(\mathrm{R}_{1}=\mathrm{OH}, \mathrm{R}_{2}=\mathrm{Ac}\right)$.

The reaction was performed in a micro tube. First, glacial acetic acid was added to sodium carbonate and stirred at ambient temperature. Then $\mathrm{NAD}^{+}$was added, the mixture heated to $90{ }^{\circ} \mathrm{C}$ and stirred until the $\mathrm{NAD}^{+}$dissolved. For dilution of the residual sodium acetate a small amount of denatured alcohol was slowly added before precipitating the product by rapid addition of more denatured alcohol. After preparation of the product mixture, two chromatographic steps were performed to yield pure isomeric compounds. At first, an ion exchange chromatography with an HPLC system was performed to separate residual $\mathrm{NAD}^{+}$from the products (Figure 3.23). This was achieved by using an aminopropyl-linked silica gel column. The chromatogram was monitored at an absorption of $254 \mathrm{~nm}$ and peaks presumably containing product were collected. Since acetic acid and sodium acetate were used as solvents, salt remained in the sample after evaporation. At 
least three desalting cycles with denatured alcohol were neccessary before the presence of metabolite in the samples could be analyzed by mass spectrometry. As final step in the purification, the products were separated by an hydrophilic interaction chromatography (HILIC) using a polyHYDROXYETHYL $A^{\mathrm{TM}}$ column (Figure 3.24). In this mode molecules are retained according to their hydrophilicity. The elution gradient contained ammonium acetate and acetonitrile. Absorption at $254 \mathrm{~nm}$ was monitored, peaks collected and analyzed by mass spectrometry.

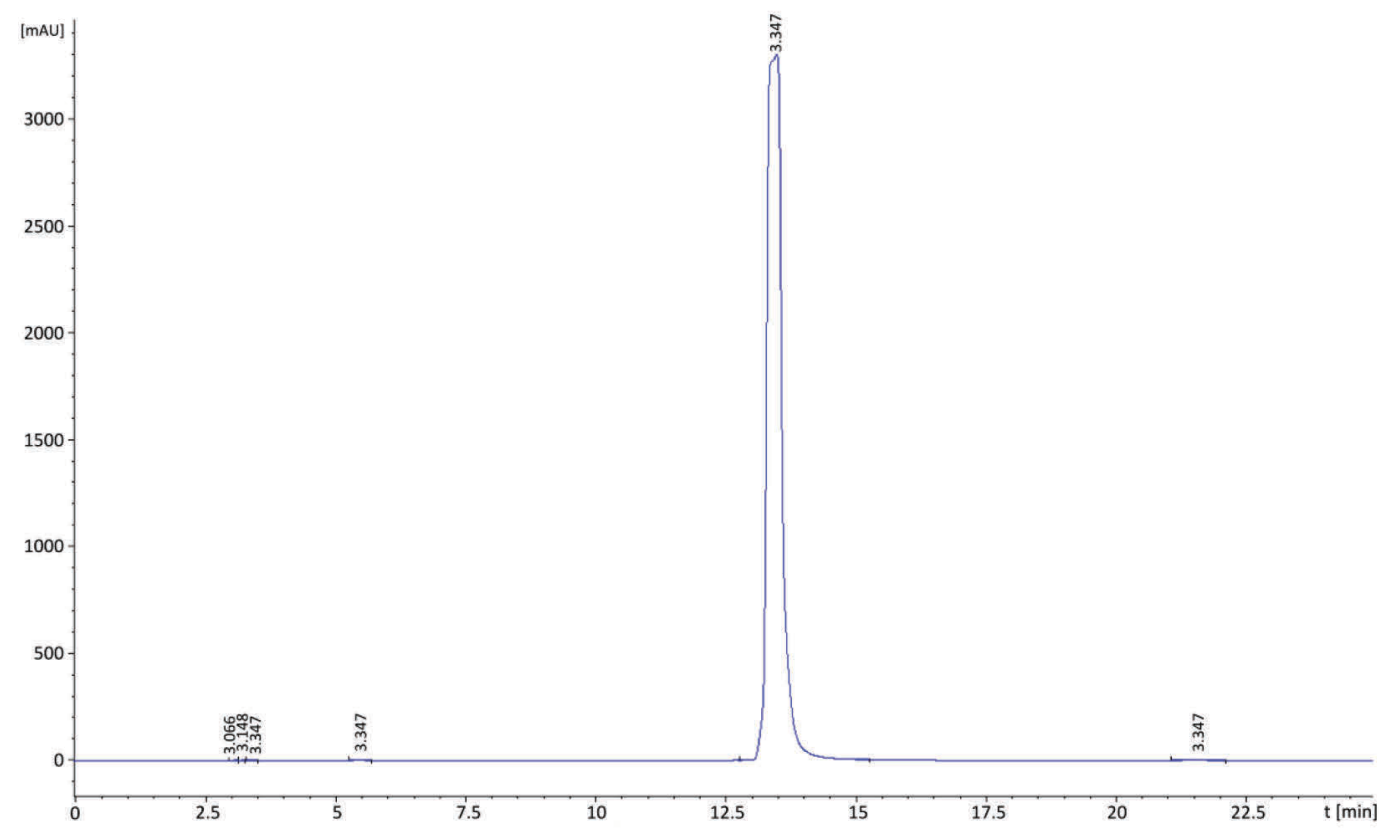

Figure 3.23: Ion exchange chromatogram of the crude reaction products from the one-step synthesis of OAADPR. The chromatography was performed using an aminopropyl-linked silica gel column and $1 \mathrm{M}$ acetic acid as buffer $\mathrm{A}$ and $1 \mathrm{M}$ sodium acetate and $1 \mathrm{M}$ acetic acid as buffer $\mathrm{B}$. The products were eluted over a separation gradient. In the peak at 13.46 min OAADPR was detected by mass spectrometry. 


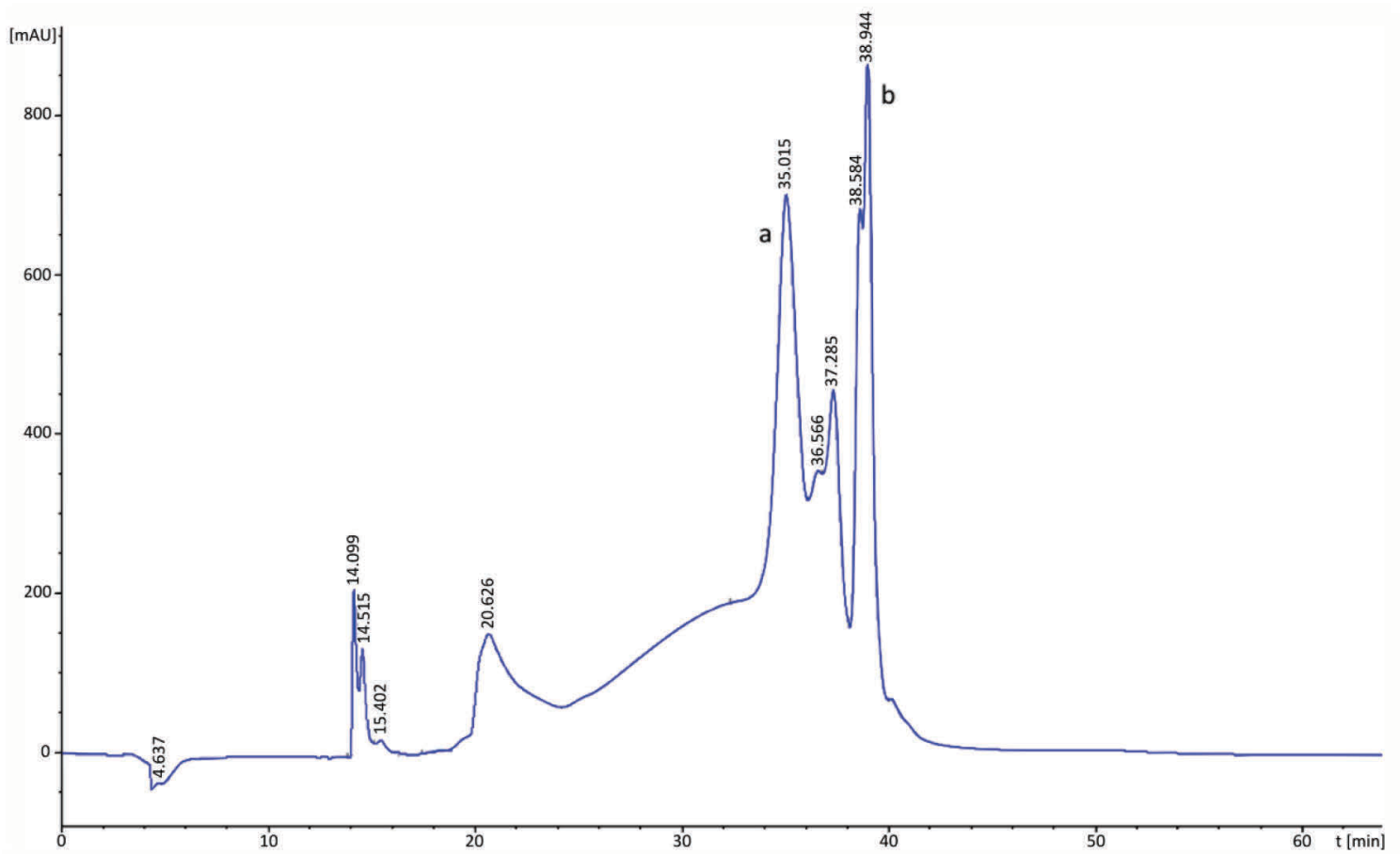

Figure 3.24: Chromatogram of the hydrophilic interaction chromatography performed as final purification step in OAADPR synthesis. $10 \mathrm{mM}$ ammonium acetate and acetonitrile were used for the separation gradient on a polyHYDROXYETHYL $A^{\text {TM }}$ column. Peaks were collected and analyzed by mass spectrometry for the presence of OAADPR. Mass spectra of the marked retention times $a$ and $b$ are shown in Figures 3.25 and 3.26 .

Figures 3.25 and 3.26 detail mass spectra of the two peaks marked as $a$ and $b$ in Figure 3.24. In spectrum a, the peak with the highest intensity possessed a mass of $600 \mathrm{Da}$, which correlates with the mass of OAADPR. The highest intensity in mass spectrum $b$ represented a peak at $558.2 \mathrm{Da}$. This is $42 \mathrm{Da}$ less than OAADPR and corresponds to the mass of ADPR.

In summary, these results detail the successful synthesis and isolation of OAADPR. 
a)

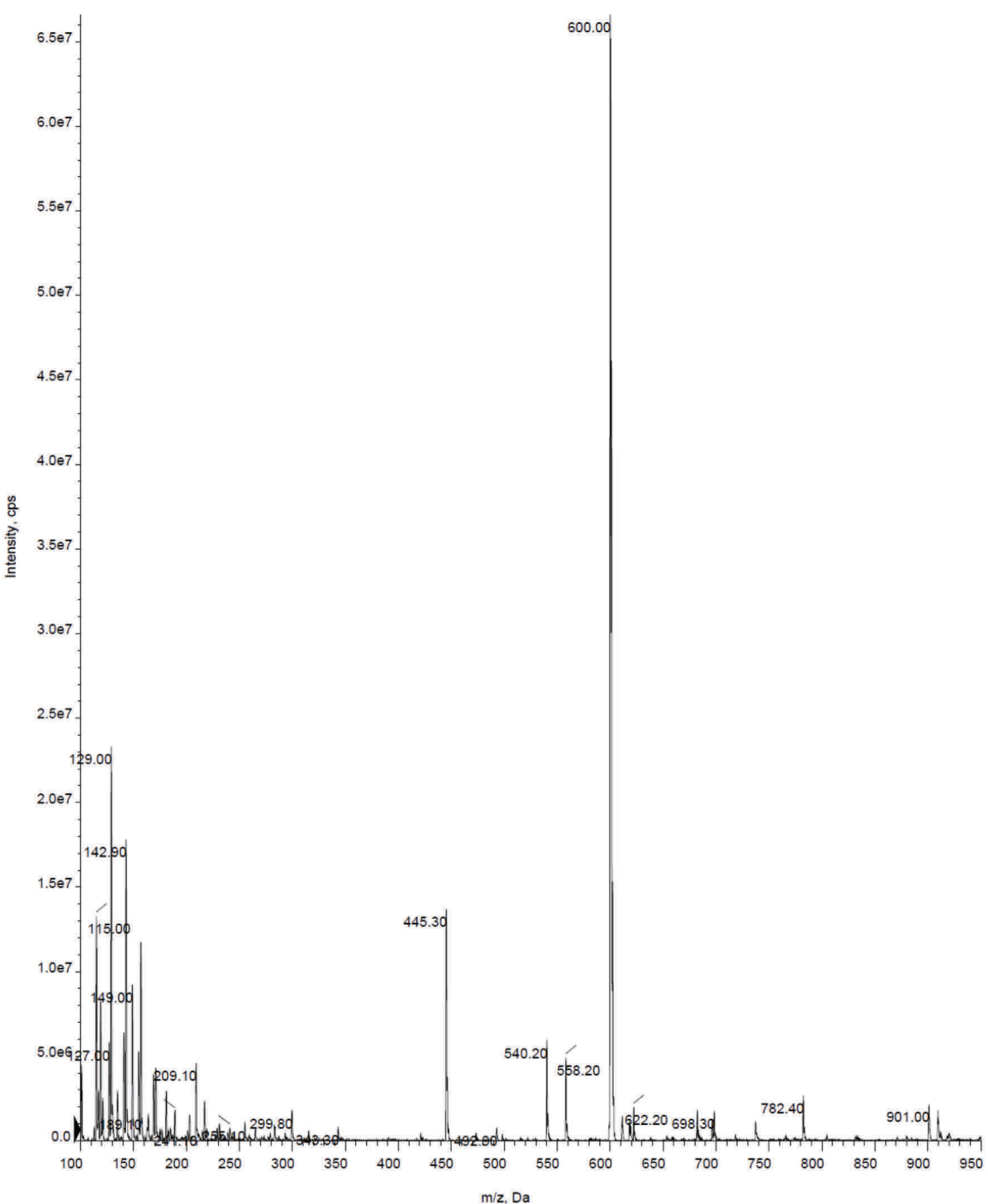

Figure 3.25: Mass spectrum of the sample from the retention time indicated in Figure 3.24. The spectrum displays the mass peak of OAADPR $(\mathrm{m} / \mathrm{z}=600 \mathrm{Da})$. 
b)

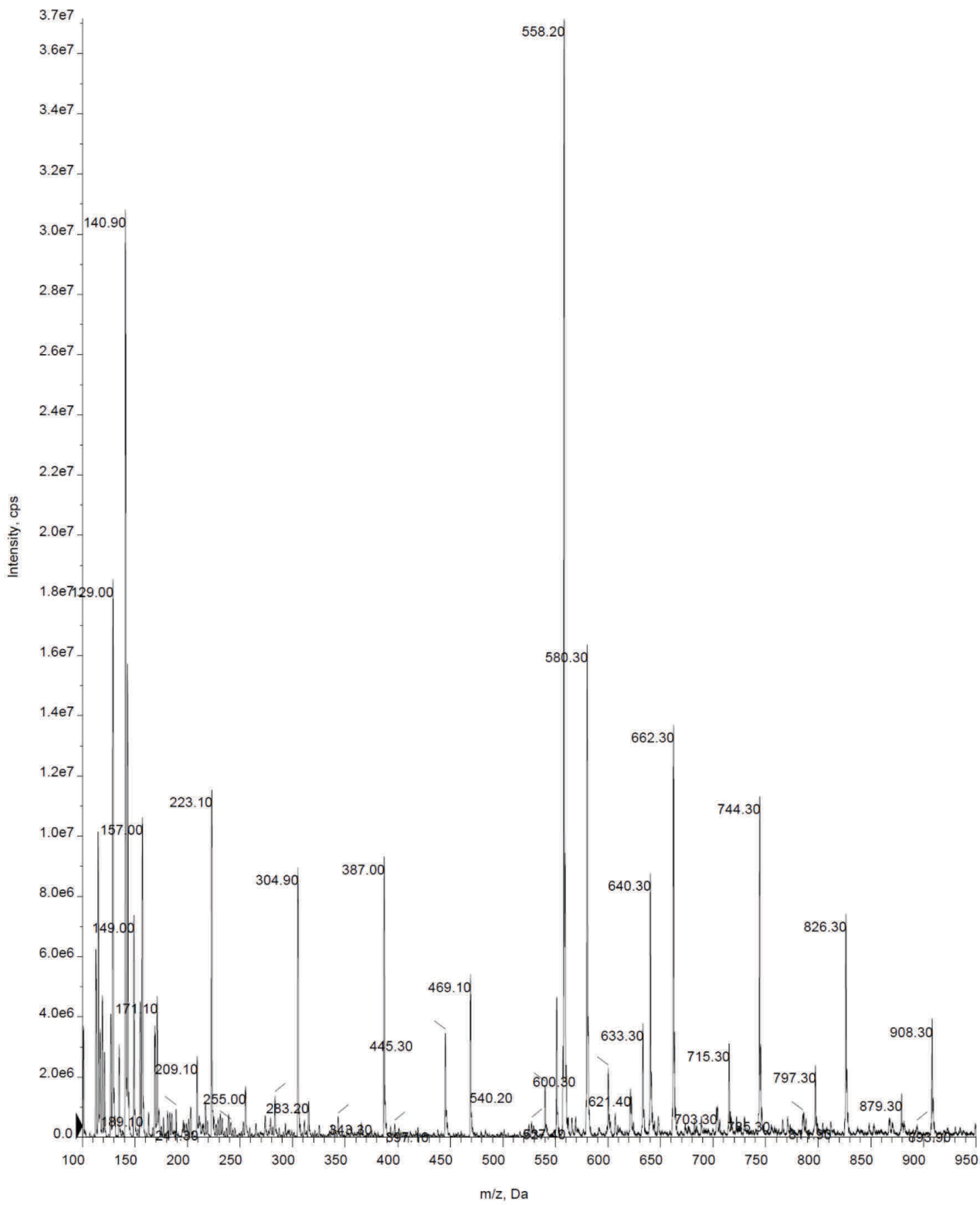

Figure 3.26: Mass spectrum of the sample from the retention time indicated in Figure 3.24. The main peak at 558 Da represents ADPR. The peaks at $662,744,826$ and 908 Da refer to remaining salt in the sample. 


\subsection{Removal of acyl modifications on histone H4K16 by sirtuins}

The following project was a collaboration with Liljan Hahn, a PhD student in the same group. The contributions are named in the different sections.

In recent years, studies have shown that the acylation of histone H4K16 is not limited to acetyl groups. Numerous acylations like butyrylation, propionylation, crotonylation myristoylation, malonylation and succinylation have been observed. ${ }^{10-12}$ Analysis of their effects on nucleosome structure and function have proven difficult, since the generation of histone $\mathrm{H} 4$ with site-specific modifications was not feasible. The protein was prone to degradation and therefore not accessible for genetic code expansion. In 2012, Bryan Wilkins solved this problem by creating a stable fusion protein (data unpublished). It consists of a shortened histone $\mathrm{H} 3$, a linker including a TEV protease cleavage site, and the histone $\mathrm{H} 4$ with an amber codon at position $\mathrm{K} 16$ for the incorporation of an unnatural amino acid (UAA) of interest via genetic code expansion.

For the integration of such an UAA with a modification into the genetic code, an additional pair of tRNA and aminoacyl-tRNA synthetase (AARS) needs to be evolved. Additionally, it needs to be targeted towards a stop codon, whose original function will be suppressed. The evolved AARS has to be specific towards the UAA and the tRNA. Since the incorporation of the UAA occurs parallel to the normal translation, it has to be orthogonal to the host's transcription machinery. The orthogonality is retained when the orthogonal synthetase does not charge endogenous tRNAs and the orthogonal tRNA cannot be recognized by endogenous sythetases. This is assured by the type of selection experiments performed while isolating a specific and orthogonal AARS from a library of mutants.

The ability to produce cleanly modified histone $\mathrm{H} 4$ allowed for the opportunity to investigate different acyl modifications at lysine 16 on this histone.

Focus of the following analysis lay on the effect of the different acyl-chain lengths of acetyl-, butyryl- and crotonyllysine on turnover kinetics by HDACs. It was asked if histone deacetylases could discriminate between different substrates and show putative specificities. 
For these experiments the sirtuin CobB was chosen as deacetylase. I cloned the plasmid and prepared the protein as described in 2.2.2.3 and 2.2.3.3. Liljan Hahn evolved the tRNA/tRNA-synthetase pairs to incorporate the acetyl, butyryl and crotonyl modifications at position $\mathrm{K} 16$ on histone $\mathrm{H} 4$ and prepared the histones $(2.2 .2 .3,2.2 .3 .3)$. In the next step, we established a lysine deacylation assay (2.2.4.5). Immunoblotting was used to monitor the cleavage of the acyl modifications over time. At first, I tested the antibodies for their specificity (Figure 3.27). It was observed, that the anti-histone H4K16ac polyclonal antibody could also detect butyrylation, but no crotonylation. The anticrotonyllysine monoclonal antibody was also able to detect butyrylation but to a smaller degree than crotonylation. It was not able to detect acetylation. In the following experiments, the anti-histone H4K16ac antibody was used to detect acetylation and butyrylation and the anti-crotonyllysine antibody to detect crotonylation.

A)

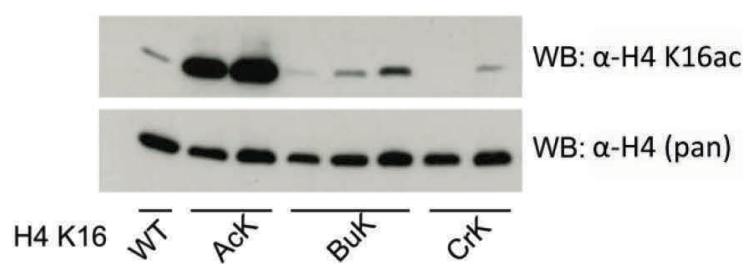

B)

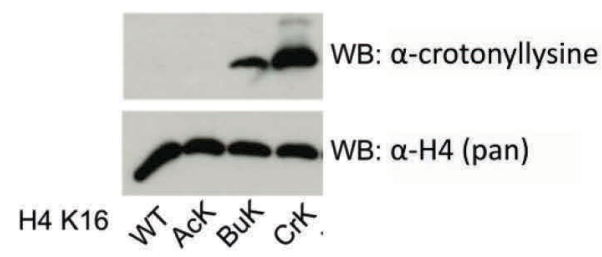

Figure 3.27: Western Blots of antibody tests with wild type histone H4 and differently acylated H4K16 histones. A) Western Blot decorated with anti-histone H4K16 ac antibody. Besides the very strong signal for acetylation, also a signal for butyrylation was visible. Crotonylation could not be detected by this antibody. B) Western Blot decorated with anti-crotonyllysine antibody. Crotonylation was recognized best by this antibody and butyrylation to a smaller degree. Wild type histone and acetylation were not recognized by this antibody.

The assays were performed with a 30-fold molar excess of modified histone compared to the deacetylase. In this way, the reaction rate was only dependent on the velocity of the enzyme and reflected its affinity for the substrate. Also the co-substrate for CobB, $N A D^{+}$, was added in excess. The reaction was started by addition of CobB to the modified histone. Then, the mixture was incubated at RT for 30 minutes. Samples were taken at different time points and quenched by the addition of preheated nicotinamide and $4 x$ 
loading buffer for SDS gels and denaturation at $95^{\circ} \mathrm{C}$ for $5 \mathrm{~min}$. The level of remaining acylation was detected by immunoblotting with the corresponding antibody and could be quantified by comparison to the total amount of protein loaded. The resulting Western Blots are depicted in Figure 3.28. They show that the reaction of CobB with acetylated H4K16 possessed a slower tunrover rate than with the butyryl or the crotonyl modification. The butyrylation was completely consumed after $4 \mathrm{~min}$, while the acetylation was still present after $10 \mathrm{~min}$. The fastest deacylation event occured on crotonylated lysine and was completed within 2 min of the reaction.

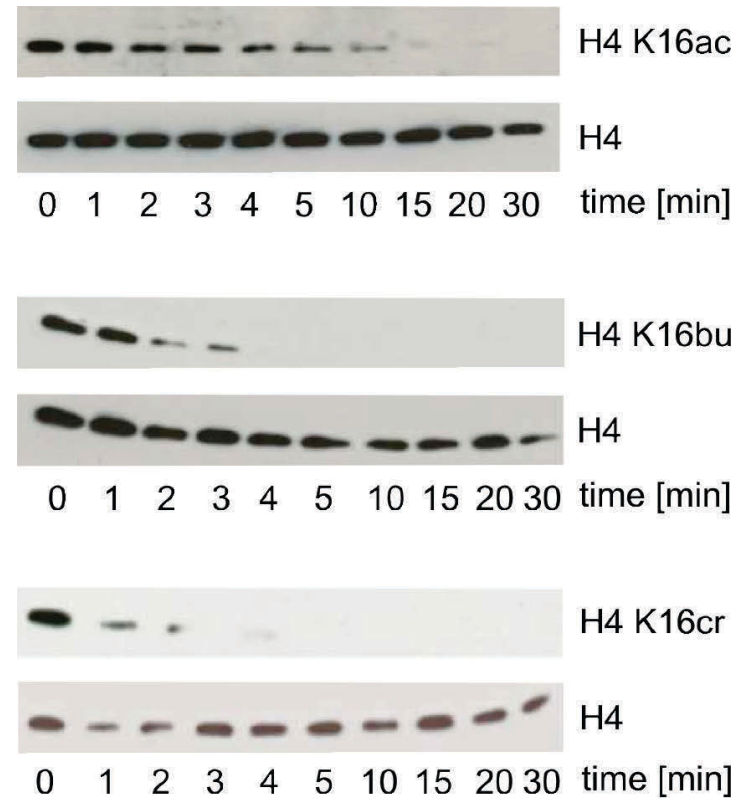

Figure 3.28: Western Blots of lysine deacetylation assay samples taken at indicated time points. The assay was performed with CobB deacetylase and histone H4 carrying different acyl modifications at position K16 as depicted. Signals in the upper sections represent acylated protein, visualized with anti-acetyl or anticrotonyllysine antibody, respectively. In the lower sections, protein was visualized with anti-H4 antibody for quantification purpose.

The same lysine deacetylation assay was also performed with the human sirtuin Sirt1. I cloned this deacetylase from human cDNA and isolated the recombinant protein from E. coli (2.2.2.32.2.3.3). The experiments were performed by Liljan Hahn and me, using the same protocol as in the assays with CobB. Figure 3.29 shows the resulting Western Blot 
signals of the butyryl modification. Sirt1 cleaves the butyryl groups within 4 min and already after $1 \mathrm{~min}$ half of the signal intensity is gone. Hence, this reaction is faster than the debutyrylation by CobB, where after $1 \mathrm{~min}$ more than half of the signal intensity remained.

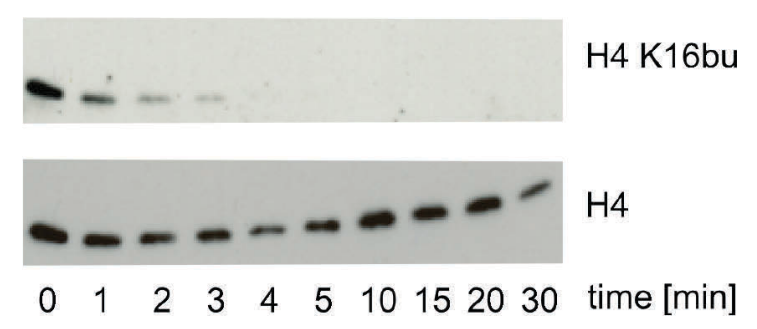

Figure 3.29: Western Blots of histone H4K16 debutyrylation by Sirt1. Consumption of the butyryl modification was completed within 4 min after the reaction was initiated. $50 \%$ was cleaved after 1 min.

The reactions of Sirt1 with acetyllysine- and crotonyllysine-modified $\mathrm{H} 4$ were not consistent within the three different measurements performed (Figure 3.30). For that reason they are regarded as preliminary results. More measurements need to be performed to obtain authentic evidence about the turnover rates of Sirt1 towards the two modifications. For the acetyl modification, one measurement shows a clearly noncomplete consumption until 30 min reaction time. In the two other measurements, the reaction velocity is somewhat faster. Overall, the turnover rate is slower than in the experiments with CobB, where the signal was cleaved after $10 \mathrm{~min}$. The measurements of decrotonylation also showed different turnover rates. In one reaction more than $50 \%$ of the signal intensity was cleaved within $2 \mathrm{~min}$ and it was completely consumed after $5 \mathrm{~min}$. The second reaction was slower and it took $10 \mathrm{~min}$ until $50 \%$ of the signal intensity was cleaved. The reaction was completed after $30 \mathrm{~min}$. In the third experiment only a marginal reduction of the crotonyllysine signal could be observed. Therefore, no conclusion can be drawn for the decrotonylation rate of Sirt1. 

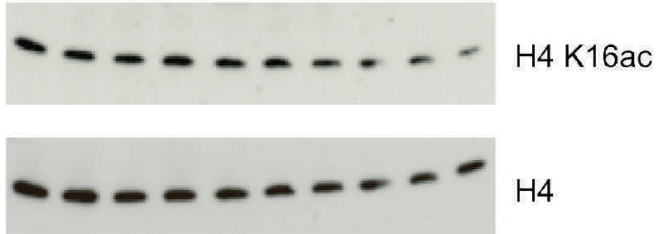

$\begin{array}{lllllllllll}0 & 1 & 2 & 3 & 4 & 5 & 10 & 15 & 2030 & \text { time }[\mathrm{min}]\end{array}$

$-\quad \ldots \ldots+\ldots$ H4 K16ac
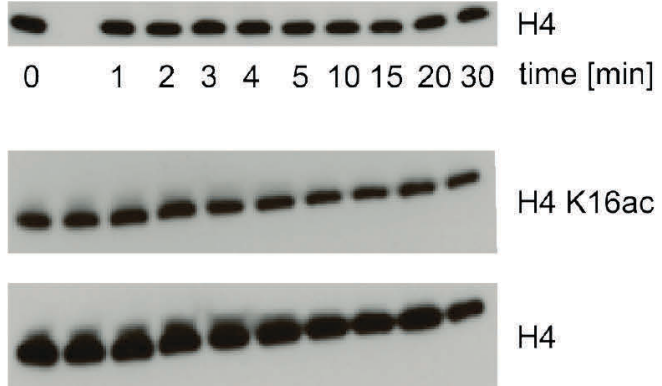

$\begin{array}{lllllllllll}0 & 1 & 2 & 3 & 4 & 5 & 10 & 15 & 20 & 30 & \text { time }[\mathrm{min}]\end{array}$

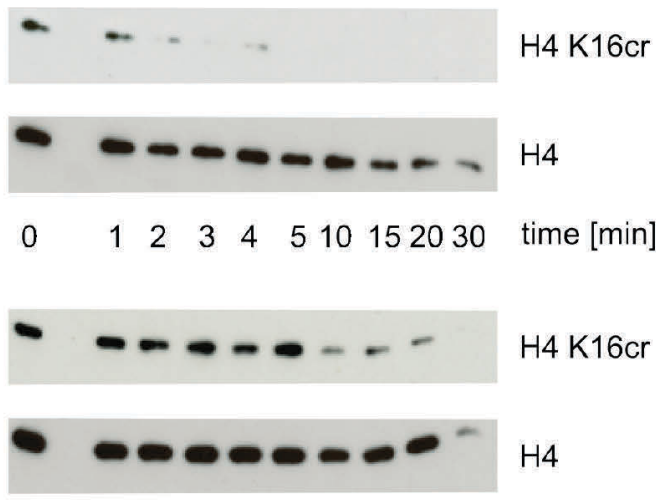

$\begin{array}{lllllllllll}0 & 1 & 2 & 3 & 4 & 5 & 10 & 15 & 2030 & \text { time [min] }\end{array}$
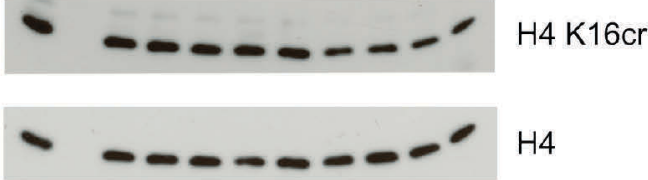

$\begin{array}{llllllllll}0 & 1 & 2 & 3 & 4 & 5 & 1015 & 2030 & \text { time [min] }\end{array}$

Figure 3.30: Western Blots of lysine deacetylation on the left and decrotonylation on the right by Sirt1. Measurements of the individual modifications are not consistent and therefore regarded as preliminary results. Cleavage of acetyl groups off histone H4K16 took either about 30 min or longer. Rates of decrotonylation were between $2 \mathrm{~min}$ and more than $30 \mathrm{~min}$. 


\section{Discussion}

\subsection{The functional optimized O-acetyl-ADP-ribose synthase 1.1 and its characterization}

A main aspect of this thesis was the engineering and optimization of an enzyme, which is capable of in situ formation of the sirtuin metabolite OAADPR. The first step in optimizing the original synthase was to determine an ideal length for the linker region connecting the deacetylase and acetyltransferase moieties to obtain enhanced activity. The peptide chain had to be long enough for the enzyme moieties to reach the substrate lysine in the linker but also short enough to ensure no hindrance and fast binding. In the original synthase OAADPRS 1.0, the peptide chain consisted of 68 amino acids. This proved to not be an optimal length, since OAADPRS 1.1, which has a five amino acid deletion in the linker region, showed a $21 \%$ higher catalytic activity. A third linker, shortened by another five amino acids to 58, led to a decrease in activity. Of the three linker lengths tested, the one with 63 amino acids, OAADPRS 1.1, proved to be the most functional. This result was reflected in the turnover numbers derived from the enzymatic coupled sirtuin assay. On this account, OAADPRS 1.1 was chosen for further characterization and following experiments.

A kinetic characterization was performed to investigate the properties of the two enzymatic moieties CobB and Hat1 in OAADPRS 1.1. First, the moiety of the sirtuin CobB was characterized in a $\mathrm{NAD}^{+}$titration assay. In general, sirtuins are slow turnover enzymes with typical deacetylation rates between 0.01 and $0.1 \mathrm{~s}^{-1} .^{81}$ Analysis of the CobB moiety yielded a turnover number of $0.04 \mathrm{~s}^{-1}$, which lies within that range. Human Sirt1, in comparison, possesses a deacetylation rate of $0.06 \mathrm{~s}^{-1}$ and yeast Sir2 one of $0.03 \mathrm{~s}^{-1} .59,82$ These values show that the turnover number of CobB, when incorporated into the synthase, was comparable to deacetylation rates of free sirtuins. The proximity of the acetyltransferase moiety Hat1 did not hamper CobB activity and the substrate lysine was 
well accessible. Substrate turnover mediated by the Hat1 moiety was determined in a second assay, where acetyl-CoA was titrated. The resulting turnover number was similar to that of CobB with a value of $0.06 \mathrm{~s}^{-1}$. Literature shows that other acetyltransferases can acetylate substrate molecules significantly faster. Gcn5, another acetyltransferase in S. cerevisiae, with histone H3 lysine 14 as primary target, has a turnover number of $1.70 \mathrm{~s}^{-1} .83$ Human Hat1 has an acetylation target, like yeast Hat1 in the synthase, on histone $\mathrm{H} 4$ lysine 12 but with a significant higher rate of $4.14 \mathrm{~s}^{-1}$. This is about 70-fold faster. ${ }^{84}$ The reason for these higher substrate turnover numbers of free acetylases might be due to known complex formations with proteins needed for the optimal function of the enzyme. While searching for the predominant cytoplasmic histone acetyltranferase activity in S. cereviviae, Parthun and coworkers found a protein that co-purified from cytosolic extracts with Hat1. The protein, called Hat2, was identified as a histone chaperon and is a homologue of mammalian Rbap46/48 proteins, which play a role in chromatin modification. It forms the HAT-B complex together with Hat1. This evolutionary conserved complex acetylates newly synthesized soluble histone $\mathrm{H} 4$ on lysine 5 and $12 .{ }^{85}$ HAT-B complexes with a catalytic subunit similar to Hat1 and a subunit similar to Hat2 have also been identified in human, Xenopus laevis and maize cell extracts. ${ }^{66,70,86-88}$ Studies with $\Delta$ hat2 strains indicated that Hat2 association is significant for full Hat1 activity by increasing the interaction between Hat1 and its substrate. ${ }^{89}$ Hat1 incorporated into the synthase has no such association and might therefore not be fully active.

A second explanation for the slow acetylation activity of the Hat1 moiety could lie in the fact that it shares its substrate with the CobB moiety. The turnover rate of CobB determines how fast the substrate lysine is deacetylated and available for acetylation by Hat1. Therefore, the acetylation rate of Hat1 cannot be significant faster than the deacetylation rate of CobB. Consistent with this assumption are the similar turnover numbers of the two enzymatic moieties. 


\subsection{Analysis of the effects of synthase 1.1 expression and OAADPR formation}

A growth test yielded initial information about the phenotype of $E$. coli cells expressing the synthase and, in turn, harbouring the metabolite OAADPR. Previous experiments in starfish oocytes and blastomeres showed a delay, or block in maturation when OAADPR was injected. ${ }^{29}$ Indeed, a significant difference of the phenotype could be observed in the growth test after induction. The cells which contained the active synthase and produced the metabolite, showed a severe delay in growth. Cells expressing the inactive synthase did not show this growth deficiency and the growth curve was similar to the control. These different phenotypes indicate that the metabolite is involved in this depression of the cellular metabolism.

In further studies with starfish oocytes, it was also observed that the injection of ADPR delayed or blocked maturation. It could not be distinguished if the observed maturation defect was a direct effect of OAADPR or of a metabolic breakdown product. In E. coli, a Nudix hydrolase, Orf209, was found to catalyze the hydrolysis of the pyrophosphate linkage in ADP-sugars and possesses high activity on ADP-ribose. This protein is functionally related to the yeast ADP-ribose hydrolase Ysal. ${ }^{90,91}$ In vitro studies revealed that Ysal cleaves OAADPR as well as ADP-ribose. ${ }^{30}$ Therefore, it might be possible that Orf209 possesses the same ability and that the growth delay seen in this growth test is triggered by the metabolic breakdown products of OAADPR. A growth test of cells with a deletion of the Orf209 gene, or with the protein inhibited, and the active synthase present would clarify if the effect is based on the metabolite itself.

The initial expression system for the synthases in E. coli used an IPTG inducible T7lac promoter on a pCDF plasmid vector. This system can be turned on by the addition of IPTG but the expression level cannot be controlled by IPTG titration. It is also known that the transcription can take place in the absence of the inducer. ${ }^{92}$ For analysis of the metabolic changes caused by the active synthase and metabolite formation, a more tightly controlled expression system was needed. The expression level should be modulated by 
inducer concentration. Hence, an expression system with the $\mathrm{P}_{\mathrm{BAD}}$ promoter of the $\operatorname{araBAD}$ operon was chosen. In the presence of the inducer arabinose, moderately high levels of expression are obtained and the promoter can be dose-dependent regulated. ${ }^{93}$ After the synthase and the inactive mutant were cloned into the new vector, analysis of expression level dependent metabolic activity and cell viability was performed. The resazurin assay was used to visualize the status of metabolic activity in the different cell cultures. In this assay, the redox dye resazurin is reduced by coenzymes like NADPH, which are indicators for the metabolic flux. The strong fluorescence signal emitted by the reduction product can be measured and metabolic activity quantified.

Overall, the results of the resazurin assay showed a direct effect of the induction level of the active synthase on metabolic activity of the cells and their viability. The more synthase was produced, the more the metabolism was depressed. Expression of the synthase triggered the formation of the metabolite. It is suspected to act as a signalling molecule for cell activity and influences metabolism, like observed in the starfish oocytes experiments. Modulated induction showed no impact on expression of the inactive synthase. Noticeably, at both induction levels, metabolic activity decreased after four hours of expression to approximately $80 \%$ of the control. From the first through the third hour it remained at $100 \%$. This might be due to effects caused by the inactive synthase, although it does not harbour a substrate lysine. It is not able to catalyze a reaction with this substrate but maybe with other lysine substrates in proximity. If this was the case, a synthase with mutations in the active sites of CobB and Hat1 would show no such decrease of metabolic activity.

Since the synthase needs $\mathrm{NAD}^{+}$as a co-substrate for catalysis, the extent of $\mathrm{NAD}^{+}$ consumption was analyzed at the two different induction levels. $N A D^{+}$is an important coenzyme in many metabolic pathways like glycolysis and the tricarboxylic acid cycle (TCA). A decrease in its availability would affect those pathways. Results of the analysis of the $N A D^{+}$levels in the cells showed a severe decrease when the active synthase was expressed and the metabolite formed. Depending on the induction level, 50 to $86 \%$ less 
$\mathrm{NAD}^{+}$could be determined in those cells compared to the control. Such a high decrease of available $\mathrm{NAD}^{+}$certainly affected the function of the mentioned metabolic pathways in the cells and their fidelity. These results are concurrent with the depression of cell metabolism measured in the resazurin assay. Expression of the inactive mutant resulted in a decrease of $\mathrm{NAD}^{+}$as well but to a lot smaller degree. As the deacetylase moiety in the inactive mutant is fully functional and no substrate lysine is present in the linker, it could still react with other acetyllysines in the cytosol. Hence, NAD ${ }^{+}$would be consumed, which would affect metabolism and explain the decreased metabolic activity measured in the resazurin assay. But the whole process would be slower than with the active synthase, since this is not an in situ reaction and deacetylase and acetyllysine must first congregate.

For a better understanding, and an overview of the affected metabolic pathways by synthase expression and metabolite formation, a microarray gene expression analysis was performed. As in analyses before, samples with the active synthase, the inactive mutant and the control were analyzed and compared. Unsurprisingly, the most regulated genes were detected in the comparison of the active synthase with the control samples. But the most eminent gene regulations were found in the survey where samples of the active synthase were compared to the ones of the inactive mutant. The activity of the synthase and the subsequent metabolite formation led to an upregulation of 34 genes and a downregulation of 40 genes. This number of regulated genes is comparable to the effects of a transcription factor. Some transcription factors like ArcA or FNR can regulate more than 150 genes and CAP more than 180 genes, while others only 20. Genes that exhibited a more than 2-fold regulation in this survey were considered to be the best candidates to inform upon the modulation of cellular pathways caused by activity of the synthase.

The highest upregulated gene was $u h p T$. It encodes a sugar phosphate transport protein in the phosphoenolpyruvate-carbohydrate phosphotransferase system (PTS). ${ }^{94}$ Expression of this gene is controlled by two transcription factors, UhpA and CAP (catabolite gene activator protein). UhpA is required for transcription activity whereas CAP stimulates the transcription and is not essential. ${ }^{95,96}$ The promoter activity is induced by extracellular 
glucose 6-phosphate (Glu6P) through an unusual two-component regulatory system, in which the products of the $u h p B$ and $u h p C$ genes regulate the activation of UhpA. ${ }^{97}$ Since the growth medium in the experiments was not supplemented with additional Glu6P, uhpT upregulation did not originate from an increased UhpA activation.

CAP, on the other hand, is activated by cyclic AMP (CAMP) through a conformational change while forming a cAMP-CAP complex, which enables CAP to bind DNA. Cellular levels of CAMP are influenced by the carbon source available in the medium. Under catabolite-repressing conditions the level of cAMP-CAP complex is low and the activity of CAP-dependent promoters decreased. As already discussed, $\mathrm{NAD}^{+}$is used as a cosubstrate by the synthase and its decreasing amount negatively influences the energy metabolism of the cell. To compensate, more carbohydrates need to be utilized and metabolism is shifted from a catabolite-respressing to a non-catabolite-repressing state. This stimulates CAMP synthesis and subsequently cAMP-CAP complex formation. The complex finally enhances uhpT transcription, which can lead to a 10 - to 15 -fold upregulation of the gene expression. ${ }^{78}$ In summary, the increased expression of uhpT is probably due to a shift in metabolism indirectly caused by OAADPR formation.

The CAMP-CAP complex dependent transcription increase was also observed for other regulated genes in the survey. One example is the gene yjiY. Expression of this gene, which encodes an inner membrane protein, was 5-fold upregulated. The gene is activated in cells utilizing amino acids or peptides as their carbon source at the beginning of the stationary-growth phase. ${ }^{98}$ Two other genes, fruB and fruK, encode proteins similar to UhpT that belong to the superfamily of the phosphotransferase system (PTS) and are controlled by the CAMP-CAP complex as well. ${ }^{99-101}$ Interestingly, fruA, also residing in the same operon, did not display a significant upregulation. A third group of CAMP-CAPdependent upregulated genes were $\operatorname{araA}, \operatorname{araB}$ and $\operatorname{araD}$. All three enzymes are located in the araBAD operon and catalyze steps in the L-arabinose degradation pathway. ${ }^{102,103}$ The experiments included the addition of L-Arabinose to the cell cultures for induction of the $\mathrm{P}_{\mathrm{BAD}}$ promoter. Although it was also present in the cultures of the control and the inactive synthase, an upregulation of those genes could only be detected in samples with the 
active synthase. Transcription of the araBAD operon is stimulated by the CAMP-CAP complex. ${ }^{104}$ Therefore, the same effect might occur as for the other genes mentioned above. Interestingly, the gene $\operatorname{araC}$ that encodes the transcription activator of the araBAD operon is not upregulated. Its transcription is activated by cancelling the AraC-dependent repression in the presence of arabinose and needs the cAMP-CAP complex as coactivator. $^{105}$

Upregulation of genes Ecs0778, nadA, nadB and pnuC can be explained by their involvement in $\mathrm{NAD}^{+}$synthesis. nadA and nadB encode enzymes in the $\mathrm{NAD}^{+}$de novo biosynthesis from L-aspartate. ${ }^{106}$ pnuC resides in the same operon as nadA but is involved in the $\mathrm{NAD}^{+}$salvage pathway. ${ }^{107}$ Regarding the results from the $\mathrm{NAD}^{+}$assay, an upregulation of these genes was expected. Due to a higher consumption of $\mathrm{NAD}^{+}$during OAADPR formation, the cell compensated for this by upregulation of its biosynthesis pathways. Since the inactive mutant of the synthase converts far less $N A D^{+}$, an upregulation of the relevant genes was not necessary.

In summary, an upregulation of genes belonging to $\mathrm{NAD}^{+}$biosynthesis was not unexpected after it was shown that the $\mathrm{NAD}^{+}$level is decreased in cells where the metabolite is formed.

The observed downregulation of the gene $g \lg P$ is not consistent with the assumption that the metabolism in cells producing OAADPR is shifted to a non-catabolite-repressing state. The glycogen phosphorylase is a distinct $\alpha$-glucan phosphorylase in $E$. coli. The activity of the glycogen phosphorylase is allosterically regulated by HPr, a PTS component which links PTS to the regulation of glycogen breakdown. The transcription is activated by CAMP-CAP. A decreased transcription of $g / g P$, as observed in cells harbouring the active synthase, leads to an accumulation of glycogen. This accumulation serves as an energy reserve and is one of the responses to nitrogen limitation. ${ }^{108,109}$

Expression of the genes garl, garR and gark was 2- to 4-fold upregulated in the presence of the metabolite. They are involved in the D-glucarate degradation pathway. In this pathway, D-glucarate is degraded to pyruvate and 2-phosphoglycerate which subsequently enter glycolysis. ${ }^{110,111}$ This utilization of this alternative carbon source could 
be due to the higher need for energy by cells harbouring the active synthase. The production of the metabolite OAADPR releases energy comparable to hydrolysis of ATP to ADP, which must be compensated. In glycolysis, 2-phosphoglycerate is normally formed by the phospho-glyceromutase from 3-phosphoglycerate. The expression of the gene gpmA, which encodes this enzyme, was 3-fold decreased in the presence of OAADPR and hence the synthesis of 2-phosphoglycerate negatively affected. Supplementation with 2phospho-glycerate from the D-glucarate degradation pathway would present an alternative source of this molecule. The reason for the downregulation of $g p m A$ might lie in observed interactions of OAADPR with enzymes in the preceding steps of glycolysis. The metabolite is proposed to modulate the activity of PGK and GAPDH, presumably to reroute energy metabolism to the pentose phosphate pathway. Hence, the repression of gpmA could be an effect of this modulation.

The biggest decrease of transcription could be observed for the gene Ecs2845, which encodes a glycosyl transferase. These transferases are involved in the biosynthesis of oligo- and polysaccharides and glycoconjugates producing glycoproteins which are involved in membrane formation including porins. Since the transcription of one of the major porins in E. coli and two other membrane proteins were decreased, a reduced biosynthesis of glycoproteins could be a consequence of this.

Proteins NupC and Gnd, whose corresponding genes were 3-fold downregulated, both favour the formation of ribose-5-phosphate. A decrease of ribose-5-phosphate formation by these enzymes can be associated with the metabolite, since the hydrolysis of it by Nudix hydrolases produces ribose-5-phosphate besides AMP. This new source might affect the regulation of native ribose-5-phosphate generating proteins.

Another gene downregulated in the presence of OAADPR is hmp. It encodes a flavohemoglobin, which obtains a nitric oxide dioxygenase activity to prevent nitric oxidedependent respiratory inhibition and cellular damage. The biosynthesis of nitric oxide is dependent on arginine and connects this to another downregulated gene, speA. The protein SpeA is an arginine decarboxylase. The decreased expression of $h m p$ would presume a low level of nitric oxide in the cell. But a repressed biosynthesis could not be 
observed and a degradation of arginine within the context of nitric oxide biosynthesis was not detected. Nitric oxide, together with superoxide, effect the activation of the transcription of sox $R$ from the soxRS superoxide response regulon. ${ }^{112}$ The protein SoxR, in turn, activates soxS transcription. ${ }^{113,114}$ This transcription was 2.5 -fold upregulated in cells with the metabolite present. An upregulation of soxS based on superoxide is not consistent with the formation OAADPR, since the metabolite is associated with decreased ROS levels in the cell. This would prevent activation of the soxRS regulon. Therefore, it can be speculated that the metabolite might also be associated with a decrease of reactive nitrogen species.

A closer look on the genes controlled by the soxRS regulon shows that one of them encodes a small regulatory RNA, which suppresses the synthesis of the outer membrane porin OmpF. ${ }^{115}$ This protein is a member of the OmpA-OmpF porin family. Another member of this family is OmpA, one of the major porins in E. coli. The corresponding gene was 4-fold downregulated together with two other membrane proteins in the samples harbouring the active synthase. Induction of the transcription of this porin occurs when the availability of nitrogen sources is limited. This indicates that the cells perform a de novo biosynthesis of nitrogen-containing compounds like amino acids and nucleotides when these nutrients are no longer available in the culture medium. The growth test of the active synthase and its inactive mutant showed that the culture with the active synthase was severely delayed in growth due to the formation of OAADPR. Cell cultures expressing the inactive mutant were growing comparable to the control. Therefore, the need of nutrients for cell growth was probably decreased in the presence of OAADPR and transcription levels of genes involved in the biosynthesis of these nutrients were downregulated.

A decrease of nutrients in the growth medium can also trigger cellular stress responses. yifE and $y j i D$, genes connected to the cellular stress response, were significantly downregulated in samples harbouring the active synthase. yifE promotes the transcription of rpoS, which is a master regulator of the general stress response. ${ }^{116}$ yjiD 
inhibits the proteolysis of rpoS. The downregulated gene typA is related to cellular stress response as well.

The adaption of cells to a decreased supply with nutrients can lead to an increase in the synthesis of the alarmone guanosine 5'-diphosphate 3'-diphosphate (ppGpp). The protein that synthesizes ppGpp is activated by binding of uncharged tRNA. The glycine tRNA synthetase encoding gene glys was downregulated in samples with the active synthase compared to samples with the inactive one. Hence, glycine tRNA could function in this case as an activator for increased ppGpp synthesis. ppGpp, in turn, positively regulates the expression of the histidine operon. Educts in the first reaction of the histidine biosynthesis are PRPP and ATP. ${ }^{117}$ PRPP is encoded by the gene prs. This gene was 3-fold downregulated when OAADPR was present in the cell. A gene that encodes a subunit of the ATP synthase, papD, was downregulated as well. This is concurrent with the assumption that a difference in cell growth with the active synthase and the inactive mutant can trigger these divergent gene regulations. The two genes $a c e A$ and $a c e B$ were significantly downregulated during OAADPR formation which can also be ascribed to the different cell growth. They encode the two key enzymes isocitrate lyase and malate synthase of the glyoxylate cycle. This cycle is a variation of the tricarboxylic acid cycle and is activated due to shortage of complex carbohydrate sources. Acetyl-CoA is used to form succinate followed by the convertion into oxalacetate. The oxalacetate enters gluconeogenesis, where it is subsequently converted into glucose.

To test the high upregulation of the $u h p T$ gene in the microarray gene expression analysis, a reporter gene assay was performed. For this purpose the promoter region of uhpT was ligated in front of the lacZ gene, which encodes ß-galactosidase. The amount of ß-galactosidase in the samples was measured in an activity test at different time points after induction. Samples harbouring the active synthase showed a slightly higher ß-galactosidase activity than samples with the inactive mutant. Activities in both samples were lower than in the control samples. This means that transcription of the uhpT-lacZ fusion gene was not enhanced in the presence of the active synthase. The obtained values did 
not reflect the results from the gene expression analysis, where a considerable increased expression of the uhpT gene could be observed when the synthase was active and hence the metabolite was formed. In the microarray gene expression analysis, the relative amount of mRNA transcribed from a certain gene is measured. The ß-galactosidase assay, on the other, hand gives feedback about the translation of that gene. In this case the translation might be hindered in some way although the transcription is performed at the same level as in the gene expression analysis. This would lead to lower ß-galactosidase activity and explain the discrepancy between the results.

For further transcription analysis, quantitative real-time PCR (qRT-PCR) was used. With this method it is possible to quantify mRNA formation and consequently DNA transcription from selected genes. Isolated RNA was transcribed into CDNA, which was quantified in real-time qPCR. Unfortunately, analysis of mRNA levels from uhpT was not successful. It was not possible to obtain clean DNA from the PCR performed on the CDNA. As a control, the same primer pair was used in a PCR with genomic E. coli DNA. The product was visible as a bright band with the right size on an agarose gel, which states that the primers were working well. Also two independent batches of cDNA from two different expressions and reversal transcriptions were used as template in case of a potential irregular expression or an error in reversal transcription but without success. Hence, it could not be determined if uhpT expression was indeed upregulated and therefore translation of ß-galactosidase hindered.

As an alternative, the cDNA of four other genes with a 2-fold upregulation were successfully analyzed with qRT-PCR. Genes nadA, nadB, pnuC and fruB were investigated and their transcription levels measured in the presence of either the active synthase and the metabolite or the inactive mutant. Transcription levels of the housekeeping gene gapA were used as a standard for constitutive gene transcription and the levels of the other genes put in relation to it. Since these are preliminary results as mentioned above, their significance is limited and should be interpreted with caution. The only significant regulation in transcription found so far was in the samples of nadB. When the active synthase was expressed in the cells, the transcription of nadB was increased. Further 
measurements using qRT-PCR would possibly help to validate the effects of the metabolite on gene transcription observed in the microarray gene expression analysis. In summary, some genes that were regulated by the formation of the metabolite in the cell were connected to energy metabolism or the compensation of the increased $\mathrm{NAD}^{+}$ consumption. Other distinct and new effects of the metabolite could not be observed in this gene expression analysis. Unfortunately, the obtained results could not be verified by further conducted experiments. Perhaps other methods analyzing cell metabolism would reveal new effects of the metabolite if they were present in this organism.

All experiments in this thesis were performed in E. coli and represent the first analyses of the role of the metabolite OAADPR in this organism. The simplicity of this bacterium served as a good platform for first investigations of possible effects of the metabolite on basic metabolic pathways in the cell. Earlier studies were performed in higher organisms like yeast and starfish. These organisms obtain a more complex cell structure and more metabolic pathways, which can be affected by the metabolite. Since only $\mathrm{NAD}^{+}-$or arabinose-related effects could be detected in $E$. Coli it would be interesting to continue the investigations in a higher organism to advance the understanding of the biological role of OAADPR. The engineered synthase can be applied to other organisms and provide in situ formation of the metabolite for analysis of possible effects. First experiments were already performed in yeast.

\subsection{Removal of acyl modifications on histone H4K16 by sirtuin CobB}

In this project I aimed to determine if the sirtuins CobB and Sirt1 could discriminate between different acyl modifications of the lysine residue at position 16 of histone $\mathrm{H} 4$ and if they show putative specificities. A group of substrates with different acyl-chain lengths were used to study potential effects of the chain length on the kinetic turnover.

Three different acylations were chosen as the substrate modification (Figure 4.1). Acetylation is a long known and well analyzed modification. On lysine 16 of histone H4 it has been shown to be important for the compaction of nucleosomes in vitro. It controlls 
the interaction between the $\mathrm{N}$-terminal tail of $\mathrm{H} 4$ and an acidic surface of neighbouring nucleosomes. ${ }^{118-120}$ The other two acylations used were butyrylation and crotonylation, which are fairly new identified lysine acylations. These modifications have not been very well characterized so far. However, it is known that HDACs can remove those acylations but detailed studies about the kinetics of these reactions are still elusive. Further understanding of the reaction kinetics and modifying enzymes could present new insights into the effects of these different lysine modifications on physiological processes.

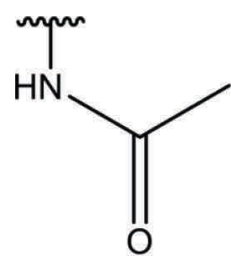

acetyllysine

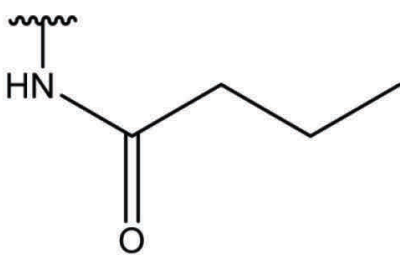

butyryllysine

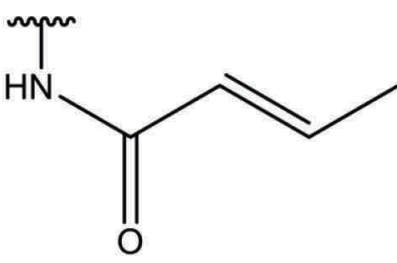

crotonyllysine

Figure 4.1: Scheme of different lysine modifications used as substrates in the deacylation assays.

Here, the cleavage of the acyl modifications over time was investigated in a newly established lysine deacylation assay. Literature states that the deacetylation of synthetic peptides, which are based on the human histone $\mathrm{H} 3$ sequence modified at lysine 14 , by other sirtuins was shown to be faster than debutyrylation. ${ }^{121}$ Therefore, it was speculated that the cleavage of acetyl groups by CobB might also be faster than the cleavage of butyryl and crotonly groups. But other than expected the fastest catalytic activity of CobB was observed when crotonyllysine was used as substrate, followed closely by butyryllysine. The deacetylation activity was decreased more than 2-fold, compared to the others. The ability of CobB to process all three different acylations on the other hand was not unexpected. Earlier experiments already showed a small discrimination ability of CobB towards its substrates. ${ }^{63}$ It was also shown that CobB has depropionylation and desuccinylation activity. ${ }^{122,123}$

The structure of the substrate binding site in CobB is apparently able to interact with lysines of different acyl-chain length and stereochemistry. A crystal structure reported by 
Zhao et al. details a cleft at the acetyllysine substrate binding site, flanked by a large Rossmann domain and a small zinc-binding domain. ${ }^{63}$ Certain interactions between the acetyllysine residue and conserved sirtuin residues in the binding site could be determined and seem to be conserved. The acetyllysine side chain lies in a hydrophobic tunnel that extends into the cleft. A hydrogen bond between the $\varepsilon$-amide of the acetyllysine and a valine of the CobB structure anchors the acetyllysine. The acetyl group does not form interactions, which might provide a reason why substrates with an extention of the acyl-chain can still bind in this tunnel. Experiments with Sir2 substrates led to the conclusion that the substrate specificity of CobB derives from regions outside the acetyllysine binding site. ${ }^{63}$ This could explain why CobB possesses the ability to process a variety of substrates including differently acylated lysines. An explanation for this spectrum of substrates might originate in the function of CobB. It activates the acetylCoA synthetase by deacetylation of an active site acetyllysine. Since it is the only known sirtuin in E. coli, it might also regulate the activity of other acyl-CoA synthetases. CobB would therefore play an important role in the control of the cellular acyl-CoA pool.

The second deacetylase analyzed was Sirt1, a human Sir2 analogue. The results for its deacetylation and decrotonylation activity are preliminary. They are not significant because the demodification rates were not consistent within the measurements. The inconsistency is probably due to the Sirt1 enzyme. Its activity seems to decrease after protein purification, probably due to protein instability. Not all assays were performed at the same timepoint and the activity of Sirt1 might have changed between assays. Therefore, it cannot be confirmed that the removal of the crotonyl group is catalyzed significantly faster than the removal of the acetyl group, like in the assays with CobB. Determination of the activity of Sirt1 by using a standard prior to peforming the assays would make it possible to classify the results.

Experiments with butyryllysine, on the other hand, generated clear results. The butyryl groups were completely cleaved within $4 \mathrm{~min}$, which is comparable to the reaction with CobB. The debutyrylation seems to be faster than the deacetylation, which took at least 
$30 \mathrm{~min}$ in all measurements for completion. These results are contradictory to analyses published by Smith and coworkers. ${ }^{121}$ An activity test of Sirt1 acting on acetyllysine and butyryllysine peptides resulted in a significant higher deacetylation activity. Their study also addressed other sirtuins. They concluded that sirtuin deacylation activity decreases with increasing chain length. These results do not concur with our experimental lysine deacylation assay. The substrate peptides used in Smith's work were synthesized as 11mers based on human histone $\mathrm{H} 3$ with the modified lysine at position 14 . The ones used in the lysine deacylation assay differ, which could be a source for the different results. Here, the full histone $\mathrm{H} 4$ from Xenopus laevis was used with an encoded acetyllysine at position 16.

In general, posttranslational modifications on histones play an important role in the regulation of a variety of cellular processes and their effects occur in a number of ways. They can alter interactions between nucleosomes or histones and the enclosing DNA to modulate chromatin structure. After the histones are modified PTM-specific binding proteins are recruited which influence downstream functions. The localization of different acyl modifications at the same sites on histones may present a new level of this regulation machinery. 


\section{Summary}

Sirtuins are a class of histone deacetylases (HDACs) found in all organisms in all three domains of life. Unlike other classes, which hydrolyze water, sirtuins require $\mathrm{NAD}^{+}$as cosubstrate and form nicotinamide and the metabolite O-acetyl-ADP-ribose (OAADPR), in addition to the deacetylated product. This metabolite is thought to function as a signalling molecule in the metabolic flux, gene expression, cellular redox processes and aging, cell cycle control and apoptosis. However, in vivo analyses of OAADPR function are hampered by its inability to permeate the plasma membrane.

The main objectives of this thesis were the engineering, optimization and characterization of an enzyme that can produce the metabolite in situ and in defined levels. This enzyme is a fusion construct that combines a sirtuin and an acetyltransferase moiety, which are connected by a peptide linker harbouring the substrate for both enzymatic reactions. The enzyme showed robust turnover of $\mathrm{NAD}^{+}$and acetyl-CoA into nicotinamide and OAADPR in an enzymatic coupled assay. This assay was dependent upon the presence of a substrate lysine in the linker. Mass spectrometry data validated the formation of OAADPR. In first analyses, effects of the metabolite on cell metabolism could be observed.

An additional objective was the analysis of turnover kinetics of the bacterial sirtuin CobB and its human analogue Sirt1 on acyl modifications of histone H4K16. The results suggest that CobB is able to process a variety of acyl modifications but with different catalytic efficiencies. It was shown that Sirt1 is able to process different acyl modifications as well, however, turnover kinetics could not yet be determined. 


\section{References}

1. Luger, K., Mäder, A. W., Richmond, R. K., Sargent, D. F. \& Richmond, T. J. Crystal structure of the nucleosome core particle at 2.8 A resolution. Nature $\mathbf{3 8 9}, \mathbf{2 5 1 - 6 0}$ (1997).

2. Marks, P. et al. Histone deacetylases and cancer: causes and therapies. Nat. Rev. Cancer 1, 194-202 (2001).

3. Allfrey, V. G., Faulkner, R. \& Mirsky, A. E. Acetylation and methylation of histones and their possible role in the regulation of RNA synthesis. Proc. Natl. Acad. Sci. U. S. A. 51, 786-94 (1964).

4. Roth, S. Y., Denu, J. M. \& Allis, C. D. Histone acetyltransferases. Annu. Rev. Biochem. 70, 81-120 (2001).

5. Calestagne-Morelli, A. \& Ausió, J. Long-range histone acetylation: biological significance, structural implications, and mechanisms. Biochem. Cell Biol. 84, 51827 (2006).

6. Nightingale, K. P., O’Neill, L. P. \& Turner, B. M. Histone modifications: signalling receptors and potential elements of a heritable epigenetic code. Curr. Opin. Genet. Dev. 16, 125-36 (2006).

7. Kurdistani, S. K., Tavazoie, S. \& Grunstein, M. Mapping global histone acetylation patterns to gene expression. Cell 117, 721-33 (2004).

8. Jazayeri, A., McAinsh, A. D. \& Jackson, S. P. Saccharomyces cerevisiae $\operatorname{Sin} 3 p$ facilitates DNA double-strand break repair. Proc. Natl. Acad. Sci. U. S. A. 101, 16449 (2004).

9. Arnaudo, A. M. \& Garcia, B. a. Proteomic characterization of novel histone posttranslational modifications. Epigenetics Chromatin 6, 24 (2013).

10. Chen, Y. et al. Lysine propionylation and butyrylation are novel post-translational modifications in histones. Mol. Cell. Proteomics 6, 812-9 (2007).

11. Tan, M. et al. Identification of 67 histone marks and histone lysine crotonylation as a new type of histone modification. Cell 146, 1016-28 (2011). 
12. Xie, Z. et al. Lysine succinylation and lysine malonylation in histones. Mol. Cell. Proteomics 11, 100-7 (2012).

13. Kouzarides, T. NEW EMBO MEMBER'S REVIEW Acetylation: a regulatory modification to rival phosphorylation ? 19, 1176-1179 (2000).

14. Dokmanovic, M., Clarke, C. \& Marks, P. a. Histone deacetylase inhibitors: overview and perspectives. Mol. Cancer Res. 5, 981-9 (2007).

15. De Ruijter, A. J. M., van Gennip, A. H., Caron, H. N., Kemp, S. \& van Kuilenburg, A. B. P. Histone deacetylases (HDACs): characterization of the classical HDAC family. Biochem. J. 370, 737-49 (2003).

16. Bjerling, P. et al. Functional Divergence between Histone Deacetylases in Fission Yeast by Distinct Cellular Localization and In Vivo Specificity Functional Divergence between Histone Deacetylases in Fission Yeast by Distinct Cellular Localization and In Vivo Specificity. Mol. Cell. Biol. 22, 2170-2181 (2002).

17. Gao, L., Cueto, M. a, Asselbergs, F. \& Atadja, P. Cloning and functional characterization of HDAC11, a novel member of the human histone deacetylase family. J. Biol. Chem. 277, 25748-55 (2002).

18. Bertos, N. R., Wang, A. H. \& Yang, X. J. Class II histone deacetylases: structure, function, and regulation. Biochem. Cell Biol. 79, 243-52 (2001).

19. Brachmann, C. B. et al. The SIR2 gene family, conserved from bacteria to humans, functions in silencing, cell cycle progression, and chromosome stability. Genes Dev. 9, 2888-2902 (1995).

20. Smith, B. C., Hallows, W. C. \& Denu, J. M. Mechanisms and molecular probes of sirtuins. Chem. Biol. 15, 1002-13 (2008).

21. Jackson, M. D. \& Denu, J. M. Structural identification of 2'- and 3'-O-acetyl-ADPribose as novel metabolites derived from the Sir2 family of beta-NAD+-dependent histone/protein deacetylases. J. Biol. Chem. 277, 18535-44 (2002).

22. Moazed, D. Enzymatic activities of Sir2 and chromatin silencing Danesh Moazed. Curr. Opin. Cell Biol. 13, 232-238 (2001). 
23. Rowen, J. The Phosphorolysis of nicotinamide riboside. J. Biol. Chem. 193, 497-507 (1951).

24. Denu, J. M. Linking chromatin function with metabolic networks: Sir2 family of NAD(+)-dependent deacetylases. Trends Biochem. Sci. 28, 41-8 (2003).

25. Tanny, J. C., Dowd, G. J., Huang, J., Hilz, H. \& Moazed, D. An enzymatic activity in the yeast Sir2 protein that is essential for gene silencing. Cell 99, 735-45 (1999).

26. Imai, S., Armstrong, C. M., Kaeberlein, M. \& Guarente, L. Transcriptional silencing and longevity protein Sir2 is an NAD-dependent histone deacetylase. Nature 403, 795-800 (2000).

27. Landry, J. et al. The silencing protein SIR2 and its homologs are NAD-dependent protein deacetylases. Proc. Natl. Acad. Sci. U. S. A. 97, 5807-11 (2000).

28. Smith, J. S. et al. A phylogenetically conserved NAD+-dependent protein deacetylase activity in the Sir2 protein family. Proc. Natl. Acad. Sci. U. S. A. 97, 6658-63 (2000).

29. Borra, M. T. et al. Conserved enzymatic production and biological effect of Oacetyl-ADP-ribose by silent information regulator 2-like NAD+-dependent deacetylases. J. Biol. Chem. 277, 12632-41 (2002).

30. Rafty, L. a, Schmidt, M. T., Perraud, A.-L., Scharenberg, A. M. \& Denu, J. M. Analysis of O-acetyl-ADP-ribose as a target for Nudix ADP-ribose hydrolases. J. Biol. Chem. 277, 47114-22 (2002).

31. Ono, T., Kasamatsu, A., Oka, S. \& Moss, J. The 39-kDa poly(ADP-ribose) glycohydrolase ARH3 hydrolyzes O-acetyl-ADP-ribose, a product of the Sir2 family of acetyl-histone deacetylases. Proc. Natl. Acad. Sci. U. S. A. 103, 316687-16691 (2006).

32. Chen, D. et al. Identification of macrodomain proteins as novel O-acetyl-ADP-ribose deacetylases. J. Biol. Chem. 286, 13261-71 (2011).

33. Sauve, A. a et al. Chemistry of gene silencing: the mechanism of NAD+-dependent deacetylation reactions. Biochemistry 40, 15456-63 (2001). 
34. Sauve, A. A. Sirtuin chemical mechanisms. Biochim. Biophys. Acta 1804, 1591-603 (2010).

35. Cen, Y. \& Sauve, A. a. Transition state of ADP-ribosylation of acetyllysine catalyzed by Archaeoglobus fulgidus Sir2 determined by kinetic isotope effects and computational approaches. J. Am. Chem. Soc. 132, 12286-98 (2010).

36. Dancy, B. C. R. et al. Azalysine analogues as probes for protein lysine deacetylation and demethylation. J. Am. Chem. Soc. 134, 5138-48 (2012).

37. Feldman, J. L., Dittenhafer-Reed, K. E. \& Denu, J. M. Sirtuin catalysis and regulation. J. Biol. Chem. 287, 42419-27 (2012).

38. Liou, G.-G., Tanny, J. C., Kruger, R. G., Walz, T. \& Moazed, D. Assembly of the SIR complex and its regulation by O-acetyl-ADP-ribose, a product of NAD-dependent histone deacetylation. Cell 121, 515-27 (2005).

39. McBryant, S. J., Krause, C. \& Hansen, J. C. Domain organization and quaternary structure of the Saccharomyces cerevisiae silent information regulator 3 protein, Sir3p. Biochemistry 45, 15941-8 (2006).

40. Rusche, L. N., Kirchmaier, A. L. \& Rine, J. The establishment, inheritance, and function of silenced chromatin in Saccharomyces cerevisiae. Annu. Rev. Biochem. 72, 481-516 (2003).

41. Chou, C.-C., Li, Y.-C. \& Gartenberg, M. R. Bypassing Sir2 and O-acetyl-ADP-ribose in transcriptional silencing. Mol. Cell 31, 650-9 (2008).

42. Martino, F. et al. Reconstitution of yeast silent chromatin: multiple contact sites and O-AADPR binding load SIR complexes onto nucleosomes in vitro. Mol. Cell 33, 323-34 (2009).

43. Grubisha, O. et al. Metabolite of SIR2 reaction modulates TRPM2 ion channel. J. Biol. Chem. 281, 14057-65 (2006).

44. Perraud, A. L., Schmitz, C. \& Scharenberg, A. M. TRPM2 Ca2+ permeable cation channels: from gene to biological function. Cell Calcium 33, 519-531 (2003). 
45. Perraud, A.-L. et al. Accumulation of free ADP-ribose from mitochondria mediates oxidative stress-induced gating of TRPM2 cation channels. J. Biol. Chem. 280, 613848 (2005).

46. Perraud, A. et al. ADP-ribose gating of the revealed by Nudix motif homology. 411, 595-599 (2001).

47. Tong, L., Lee, S. \& Denu, J. M. Hydrolase regulates NAD+ metabolites and modulates cellular redox. J. Biol. Chem. 284, 11256-66 (2009).

48. Zharova, T. V \& Vinogradov, A. D. A competitive inhibition of the mitochondrial NADH-ubiquinone oxidoreductase (complex I) by ADP-ribose. Biochim. Biophys. Acta 1320, 256-64 (1997).

49. Grivennikova, V. G. \& Vinogradov, A. D. Generation of superoxide by the mitochondrial Complex I. Biochim. Biophys. Acta 1757, 553-61 (2006).

50. Shenton, D. et al. Global translational responses to oxidative stress impact upon multiple levels of protein synthesis. J. Biol. Chem. 281, 29011-21 (2006).

51. Shenton, D. \& Grant, C. M. Protein S-thiolation targets glycolysis and protein synthesis in response to oxidative stress in the yeast Saccharomyces cerevisiae. Biochem. J. 374, 513-9 (2003).

52. Ralser, M. et al. Dynamic rerouting of the carbohydrate flux is key to counteracting oxidative stress. J. Biol. 6, 10 (2007).

53. Juhnke, H., Krems, B., Kötter, P. \& Entian, K. D. Mutants that show increased sensitivity to hydrogen peroxide reveal an important role for the pentose phosphate pathway in protection of yeast against oxidative stress. Mol. Gen. Genet. 252, 456-64 (1996).

54. Winyard, P. G., Moody, C. J. \& Jacob, C. Oxidative activation of antioxidant defence. Trends Biochem. Sci. 30, 453-61 (2005).

55. Jamieson, D. J. Oxidative stress responses of the yeast Saccharomyces cerevisiae. Yeast 14, 1511-27 (1998).

56. Comstock, L. R. \& Denu, J. M. Synthesis and biochemical evaluation of O-acetylADP-ribose and N-acetyl analogs. Org. Biomol. Chem. 5, 3087-91 (2007). 
57. Tong, L. \& Denu, J. M. Function and metabolism of sirtuin metabolite O-acetyl-ADPribose. Biochim. Biophys. Acta 1804, 1617-25 (2010).

58. Mullis, K. B. \& Faloona, F. A. [21] Specific synthesis of DNA in vitro via a polymerase-catalyzed chain reaction. Methods Enzymol. 155, 335-350 (1987).

59. Smith, B. C., Hallows, W. C. \& Denu, J. M. A continuous microplate assay for sirtuins and nicotinamide-producing enzymes. Anal. Biochem. 394, 101-9 (2009).

60. Tsang, A. W. \& Escalante-Semerena, J. C. CobB, a New Member of the SIR2 Family of Eucaryotic Regulatory Proteins, Is Required to Compensate for the Lack of Nicotinate Mononucleotide:5,6-Dimethylbenzimidazole Phosphoribosyltransferase Activity in cobT Mutants during Cobalamin Biosynthesis in Salm. J. Biol. Chem. 273, 31788-31794 (1998).

61. Starai, V. J., Celic, I., Cole, R. N., Boeke, J. D. \& Escalante-Semerena, J. C. Sir2dependent activation of acetyl-CoA synthetase by deacetylation of active lysine. Science 298, 2390-2 (2002).

62. Starai, V. J., Takahashi, H., Boeke, J. D. \& Escalante-Semerena, J. C. Short-chain fatty acid activation by acyl-coenzyme A synthetases requires SIR2 protein function in Salmonella enterica and Saccharomyces cerevisiae. Genetics 163, 545-55 (2003).

63. Zhao, K., Chai, X. \& Marmorstein, R. Structure and substrate binding properties of cobB, a Sir2 homolog protein deacetylase from Escherichia coli. J. Mol. Biol. 337, 731-41 (2004).

64. Zhao, K., Chai, X. \& Marmorstein, R. Structure of the Yeast Hst2 Protein Deacetylase in Ternary Complex with 2'-O-Acetyl ADP Ribose and Histone Peptide. Structure 11, 1403-1411 (2003).

65. Richman, R., Chicoine, L. G., Collini, M. P., Cook, R. G. \& Allis, C. D. Micronuclei and the cytoplasm of growing Tetrahymena contain a histone acetylase activity which is highly specific for free histone H4. J. Cell Biol. 106, 1017-26 (1988).

66. Verreault, A., Kaufman, P. D., Kobayashi, R. \& Stillman, B. Nucleosomal DNA regulates the core-histone-binding subunit of the human Hat1 acetyltransferase. Curr. Biol. 8, 96-108 (1998). 
67. Ruiz-Garcia, A. B. HAT1 and HAT2 Proteins Are Components of a Yeast Nuclear Histone Acetyltransferase Enzyme Specific for Free Histone H4. J. Biol. Chem. 273, 12599-12605 (1998).

68. Poveda, A. et al. Hif1 is a component of yeast histone acetyltransferase B, a complex mainly localized in the nucleus. J. Biol. Chem. 279, 16033-43 (2004).

69. Ai, X. \& Parthun, M. R. The nuclear Hat1p/Hat2p complex: a molecular link between type B histone acetyltransferases and chromatin assembly. Mol. Cell 14, 195-205 (2004).

70. Lusser, A. et al. Analysis of the histone acetyltransferase B complex of maize embryos. Nucleic Acids Res. 27, 4427-35 (1999).

71. Benson, L. J. et al. Properties of the type B histone acetyltransferase Hat1: H4 tail interaction, site preference, and involvement in DNA repair. J. Biol. Chem. 282, 836-42 (2007).

72. Qin, S. \& Parthun, M. R. Recruitment of the Type B Histone Acetyltransferase Hat1p to Chromatin Is Linked to DNA Double-Strand Breaks Recruitment of the Type B Histone Acetyltransferase Hat1p to Chromatin Is Linked to DNA Double-Strand Breaks. Mol. Cell. 26, 3649-3658 (2006).

73. Sobel, R. E., Cook, R. G., Perry, C. A., Annunziato, A. T. \& Allis, C. D. Conservation of deposition-related acetylation sites in newly synthesized histones H3 and H4. Proc. Natl. Acad. Sci. U. S. A. 92, 1237-41 (1995).

74. Rossmann, M. G. \& Argos, P. The taxonomy of binding sites in proteins. Mol. Cell. Biochem. 21, 161-82 (1978).

75. Sauve, A. a, Wolberger, C., Schramm, V. L. \& Boeke, J. D. The biochemistry of sirtuins. Annu. Rev. Biochem. 75, 435-65 (2006).

76. Dutnall, R. N., Tafrov, S. T., Sternglanz, R. \& Ramakrishnan, V. Structure of the histone acetyltransferase Hat1: a paradigm for the GCN5-related Nacetyltransferase superfamily. Cell 94, 427-38 (1998). 
77. Vega-Avila, E. \& Pugsley, M. K. An overview of colorimetric assay methods used to assess survival or proliferation of mammalian cells. Proc. West. Pharmacol. Soc. 54, 10-4 (2011).

78. Chen, Q. \& Kadner, R. J. Effect of altered spacing between uhpT promoter elements on transcription activation. J. Bacteriol. 182, 4430-6 (2000).

79. Kasamatsu, A. et al. Hydrolysis of O-acetyl-ADP-ribose isomers by ADPribosylhydrolase 3. J. Biol. Chem. 286, 21110-7 (2011).

80. Szczepankiewicz, B. G., Koppetsch, K. J. \& Perni, R. B. One-step, nonenzymatic synthesis of O-acetyl-ADP-ribose and analogues from NAD and carboxylates. J. Org. Chem. 76, 6465-74 (2011).

81. French, J. B., Cen, Y. \& Sauve, A. A. Plasmodium falciparum Sir2 is an NAD+dependent deacetylase and an acetyllysine-dependent and acetyllysineindependent NAD+ glycohydrolase. Biochemistry 47, 10227-39 (2008).

82. Sauve, A. A. \& Schramm, V. L. Sir2 regulation by nicotinamide results from switching between base exchange and deacetylation chemistry. Biochemistry $\mathbf{4 2}$, 9249-56 (2003).

83. Tanner, K. G., Langer, M. R., Kim, Y. \& Denu, J. M. Kinetic mechanism of the histone acetyltransferase GCN5 from yeast. J. Biol. Chem. 275, 22048-55 (2000).

84. Wu, H. et al. Structural basis for substrate speci fi city and catalysis of human histone acetyltransferase 1. Proc. Natl. Acad. Sci. U. S. A. 109, 8925-8930 (2012).

85. Verzijlbergen, K. F. et al. A barcode screen for epigenetic regulators reveals a role for the NuB4/HAT-B histone acetyltransferase complex in histone turnover. PLoS Genet. 7, e1002284 (2011).

86. Chang, L. et al. Histones in transit: cytosolic histone complexes and diacetylation of H4 during nucleosome assembly in human cells. Biochemistry 36, 469-80 (1997).

87. Eberharter, A., Lechner, T., Goralik-Schramel, M. \& Loidl, P. Purification and characterization of the cytoplasmic histone acetyltransferase B of maize embryos. FEBS Lett. 386, 75-81 (1996). 
88. Imhof, A. \& Wolffe, A. P. Purification and properties of the Xenopus Hat1 acetyltransferase: association with the 14-3-3 proteins in the oocyte nucleus. Biochemistry 38, 13085-93 (1999).

89. Parthun, M. R., Widom, J. \& Gottschling, D. E. The major cytoplasmic histone acetyltransferase in yeast: links to chromatin replication and histone metabolism. Cell 87, 85-94 (1996).

90. Bessman, M. J., Frick, D. N. \& O'Handley, S. F. The MutT proteins or 'Nudix' hydrolases, a family of versatile, widely distributed, 'housecleaning' enzymes. J. Biol. Chem. 271, 25059-62 (1996).

91. Dunn, C. a., O'Handley, S. F., Frick, D. N. \& Bessman, M. J. Studies on the ADPribose pyrophosphatase subfamily of the nudix hydrolases and tentative identification of $\operatorname{trgB}$, a gene associated with tellurite resistance. J. Biol. Chem. 274, 32318-24 (1999).

92. Penumetcha, P. et al. Improving the Lac System for Synthetic Biology. Bios 81, 7-15 (2010).

93. Guzman, L. M., Belin, D., Carson, M. J. \& Beckwith, J. Tight regulation, modulation, and high-level expression by vectors containing the arabinose PBAD promoter. J. Bacteriol. 177, 4121-30 (1995).

94. Maloney, P. C., Ambudkar, S. V, Anatharam, V., Sonna, L. a \& Varadhachary, A. Anion-exchange mechanisms in bacteria. Microbiol. Rev. 54, 1-17 (1990).

95. Shattuck-Eidens, D. M. \& Kadner, R. J. Molecular cloning of the uhp region and evidence for a positive activator for expression of the hexose phosphate transport system of Escherichia coli. J. Bacteriol. 155, 1062-70 (1983).

96. Merkel, T. J., Dahl, J. L., Ebright, R. H. \& Kadner, R. J. Transcription activation at the Escherichia coli uhpT promoter by the catabolite gene activator protein. J. Bacteriol. 177, 1712-8 (1995).

97. Verhamme, D. T., Arents, J. C., Postma, P. W., Crielaard, W. \& Hellingwerf, K. J. Glucose-6-phosphate-dependent phosphoryl flow through the Uhp twocomponent regulatory system. Microbiology 147, 3345-52 (2001). 
98. Kraxenberger, T., Fried, L., Behr, S. \& Jung, K. First insights into the unexplored twocomponent system YehU/YehT in Escherichia coli. J. Bacteriol. 194, 4272-84 (2012).

99. Reizer, J., Reizer, A., Kornberg, H. L. \& Saier, M. H. Sequence of the fruB gene of Escherichia coli encoding the diphosphoryl transfer protein (DTP) of the phosphoenolpyruvate: sugar phosphotransferase system. FEMS Microbiol. Lett. 118, 159-62 (1994).

100. Kornberg, H. L. Routes for fructose utilization by Escherichia coli. J. Mol. Microbiol. Biotechnol. 3, 355-9 (2001).

101. Tchieu, J. H., Norris, V., Edwards, J. S. \& Saier, M. H. The complete phosphotransferase system in Escherichia coli. J. Mol. Microbiol. Biotechnol. 3, 329-46 (2001).

102. Banerjee, S., Anderson, F. \& Farber, G. K. The evolution of sugar isomerases. Protein Eng. 8, 1189-95 (1995).

103. Johnson, A. E. \& Tanner, M. E. Epimerization via carbon-carbon bond cleavage. Lribulose-5-phosphate 4-epimerase as a masked class II aldolase. Biochemistry 37, 5746-54 (1998).

104. Lichenstein, H. S., Hamilton, E. P. \& Lee, N. Repression and catabolite gene activation in the araBAD operon. J. Bacteriol. 169, 811-22 (1987).

105. Hamilton, E. P. \& Lee, N. Three binding sites for AraC protein are required for autoregulation of araC in Escherichia coli. Proc. Natl. Acad. Sci. U. S. A. 85, 1749-53 (1988).

106. Seifert, J. et al. Expression of the E. coli nadB gene and characterization of the gene product L-aspartate oxidase. Biol. Chem. Hoppe. Seyler. 371, 239-48 (1990).

107. Sauer, E., Merdanovic, M., Mortimer, A. P., Bringmann, G. \& Reidl, J. PnuC and the utilization of the nicotinamide riboside analog 3-aminopyridine in Haemophilus influenzae. Antimicrob. Agents Chemother. 48, 4532-41 (2004). 
108. Alonso-Casajús, N. et al. Glycogen phosphorylase, the product of the glgP Gene, catalyzes glycogen breakdown by removing glucose units from the nonreducing ends in Escherichia coli. J. Bacteriol. 188, 5266-72 (2006).

109. Fung, T., Kwong, N., Zwan, T. Van Der \& Wu, M. Residual Glycogen Metabolism in Escherichia coli is Specific to the Limiting Macronutrient and Varies During Stationary Phase. J. Exp. Microbiol. Immunol. 17, 83-87 (2013).

110. Hubbard, B. K., Koch, M., Palmer, D. R., Babbitt, P. C. \& Gerlt, J. a. Evolution of enzymatic activities in the enolase superfamily: characterization of the (D)glucarate/galactarate catabolic pathway in Escherichia coli. Biochemistry 37, 14369-75 (1998).

111. Monterrubio, R., Baldoma, L., Obradors, N., Aguilar, J. \& Badia, J. A common regulator for the operons encoding the enzymes involved in D-galactarate, Dglucarate, and D-glycerate utilization in Escherichia coli. J. Bacteriol. 182, 2672-4 (2000).

112. Demple, B. Signal transduction by nitric oxide in cellular stress responses. Mol. Cell. Biochem. 234-235, 11-8 (2002).

113. Amábile-Cuevas, C. F. \& Demple, B. Molecular characterization of the soxRS genes of Escherichia coli: two genes control a superoxide stress regulon. Nucleic Acids Res. 19, 4479-84 (1991).

114. Nunoshiba, T., Hidalgo, E., Amábile Cuevas, C. F. \& Demple, B. Two-stage control of an oxidative stress regulon: the Escherichia coli SoxR protein triggers redoxinducible expression of the soxS regulatory gene. J. Bacteriol. 174, 6054-60 (1992).

115. Greenberg, J. T., Monach, P., Chou, J. H., Josephy, P. D. \& Demple, B. Positive control of a global antioxidant defense regulon activated by superoxide-generating agents in Escherichia coli. Proc. Natl. Acad. Sci. U. S. A. 87, 6181-5 (1990).

116. Baev, M. V, Baev, D., Radek, A. J. \& Campbell, J. W. Growth of Escherichia coli MG1655 on LB medium: determining metabolic strategy with transcriptional microarrays. Appl. Microbiol. Biotechnol. 71, 323-8 (2006). 
117. Alifano, P. et al. Histidine biosynthetic pathway and genes: structure, regulation, and evolution. Microbiol. Rev. 60, 44-69 (1996).

118. Wilkins, B. J. et al. A cascade of histone modifications induces chromatin condensation in mitosis. Science 343, 77-80 (2014).

119. Robinson, P. J. J. et al. $30 \mathrm{~nm}$ chromatin fibre decompaction requires both H4-K16 acetylation and linker histone eviction. J. Mol. Biol. 381, 816-25 (2008).

120. Shogren-Knaak, M. et al. Histone H4-K16 acetylation controls chromatin structure and protein interactions. Science 311, 844-7 (2006).

121. Smith, B. C. \& Denu, J. M. Acetyl-lysine analog peptides as mechanistic probes of protein deacetylases. J. Biol. Chem. 282, 37256-65 (2007).

122. Garrity, J., Gardner, J. G., Hawse, W., Wolberger, C. \& Escalante-Semerena, J. C. NIysine propionylation controls the activity of propionyl-CoA synthetase. J. Biol. Chem. 282, 30239-45 (2007).

123. Colak, G. et al. Identification of lysine succinylation substrates and the succinylation regulatory enzyme CobB in Escherichia coli. Mol. Cell. Proteomics 12, 3509-20 (2013). 


\section{Supplementry}

\subsection{Table of figures}

Figure 1.1: Schematic structure of a nucleosomal array. The DNA (black) is wrapped around the four core histones which are present in two copies. The N-terminal tails of the histones (rose) are oriented out of the octamer.

Figure 1.2: Proposed catalytic mechanism of OAADPR formation. At first the oxygen of the acetyllysine attacks the $\mathrm{C1}^{\prime}$ atom at the nicotinamide ribose and nicotinamide is released. The hydroxyl group at $\mathrm{C2}^{\prime}$ gets activated by a conserved histidine, attacks the carbon atom of the $O$-alkylamidate and a $1^{\prime}, 2^{\prime}$-cyclic intermediate is formed. This intermediate is attacked by a base-activated water molecule which leads to the formation of 2'-OAADPR and the release of deacetylated lysine. A non-enzymatic transesterification yields a mixture of 2'- and 3'-OAADPR.

Figure 1.3: Proposed mechanisms of OAADPR/ADPR and Ysal function in metabolic pathways and cellular redox. OAADPR/ADPR are generated by $\mathrm{NAD}^{+}$cleavage and hydrolyzed by Ysal to AMP which activates glycolysis. In $\triangle y s a 1$ cells OAADPR/ADPR levels are increased and consequently inhibit glycolysis and promote NADPH production by rerouting glucose to the pentose phosphate pathway. This leads to higher antioxidative stress response capability because essential cellular antioxidants can be reduced by NADPH. Increased OAADPR/ADPR levels also inhibit complex I of the electron transport chain leading to a lower ROS level.

Figure 2.1: Size exclusion chromatogram of affinity tag purified CobB using a Superdex 200 26/70 column. The synthase eluted with a size of $26 \mathrm{kDa}$. The $12 \%$ SDS gel displays CobB after the hexahistidine tag affinity purification (C) and fractions from the chromatogram. (P: cell pellet, F: flowthrough from hexahistidine tag affinity purification). 33

Figure 2.2: Size exclusion chromatogram of affinity tag purified Sirt1 using a Superdex 200 26/70 column. The synthase eluted with a size of $49 \mathrm{kDa}$. The $12 \%$ SDS gel 
displays Sirt1 after the hexahistidine tag affinity purification (C) and fractions from the chromatogram. (P: cell pellet, F: flowthrough from hexahistidine tag affinity purification). 34

Figure 2.3: Enzymatic coupled sirtuin assay. An acetyltransferase loads the lysine residue with an acetyl group from acetyl-CoA. Nicotinamide is cleaved off $\mathrm{NAD}^{+}$and the acetyl group transferred from the substrate lysine to the remaining ADP-ribose. The deacetylated lysine residue can be loaded again by the acetyltransferase. Nicotinamide is converted to nicotinic acid and ammonium whereas ammonium serves as a substrate in the photometrically traceable reaction. NADPH is oxidized to $\mathrm{NADP}^{+}$by glutamate dehydrogenase while ammonia and $\alpha$-ketoglutarate are converted to glutamate. ${ }^{58}$ The absorption maximum of NADPH lies at $340 \mathrm{~nm}$. 37

Figure 2.4: Reaction of resazurin (7-hydroxy-10-oxidophenoxazin-10-ium-3-one) to resorufin by acceptance of electrons from NADH which is oxidized to NAD ${ }^{+}$. 38

Figure 2.5: Enzymatic coupled assay for analysis of pncA-MBP activity. 39

Figure 3.1: Scheme of the $O$-acetyl-ADP-ribose synthase (OAADPR) describing its individual parts. The histone $\mathrm{H} 3 / \mathrm{H} 4$ peptide chain harbours the substrate lysine and links the catalytic core domain of the deacetylase CobB (PDB-ID: 1S5P ${ }^{62}$ ) on the left with the domain of the acetyltransferase Hat1 (PDB-ID: $1 \mathrm{BOB}^{75}$ ) on the right. In the binding cleft of CobB its co-substrate $\mathrm{NAD}^{+}$is depicted and a structral zinc ion in the zinc-binding module. The substrate of Hat1, acetyl-CoA, is bound in a cavity on the surface of the Hat1 core domain. The structure was composed using PyMOL. 45

Figure 3.2: Function of the OAADPR synthase and formation of the metabolite OAADPR. Following the release of nicotinamide, CobB relocates the acetyl group from the substate lysine to $\mathrm{NAD}^{+}$. There it finally binds to $\mathrm{C2}^{\prime}$ of the nicotinamide ribose forming OAADPR. At last the acetyltransferase Hat1 reacetylates the substrate lysine by transfer of an acetyl group from acetyl-CoA. Products of one reaction cycle are one molecule of OAADPR, nicotinamide and CoA, respectively. 46 
Figure 3.3: Size exclusion chromatogram of the affinity tag purified synthase 1.0 using a Superdex 200 26/70 column. The synthase eluted with a size of $70 \mathrm{kDa}$ after $162 \mathrm{~min}$. After $191 \mathrm{~min}$ CobB truncation of the synthase eluted from the column._47

Figure 3.4: $12 \%$ SDS gel showing OAADPRS 1.0 after hexahistidine tag affinity purification (C) and fractions from chromatogram (Figure 3.3) after size exclusion chromatography. Bands at $70 \mathrm{kDa}$ represent the full length protein and at $28 \mathrm{kDa}$ CobB truncations. 48

Figure 3.5: Scheme of the enzymatic coupled sirtuin assay. Nicotinamide is cleaved from $\mathrm{NAD}^{+}$and the acetyl group is transferred from the substrate lysine to the remaining ADP-ribose structure of $\mathrm{NAD}^{+}$to form OAADPR. The deacetylated lysine residue is charged again with an acetyl group by the acetyltransferase. Nicotinamide is converted to nicotinic acid and ammonium, whereas ammonium serves as a substrate in the photometrically detectable reaction. Then, NADPH is oxidized to $\mathrm{NADP}^{+}$by glutamate dehydrogenase while ammonia and $\alpha$-ketoglutarate are converted to glutamate. The absorption maximum of NADPH lies at $340 \mathrm{~nm}$. 49

Figure 3.6: Enzymatic coupled sirtuin assay with synthase 1.0. Arrows indicate the time points of $\mathrm{NAD}^{+}$and acetyl-CoA addition. A decrease of absorption after $\mathrm{NAD}^{+}$ addition was due to nicotinamide contamination. OAADPRS 1.0 activity was dependent on the addition of acetyl-CoA since a significant decrease of the absorption was only obtained after its addition. The negative control, containing buffer instead of synthase, did not show absorption decrease upon acetyl-CoA addition. 50

Figure 3.7: Schemes of the two modified $O$-acetyl-ADP-ribose synthases. Deleted regions are marked in red. Synthase 1.1 was shortened by five amino acids in the linker sequence. In synthase 1.2 ten amino acids of the linker sequence were deleted. _ 51

Figure 3.8: Measurement of the activity of the three different synthases with the enzymatic coupled sirtuin assay. The diagram shows the decrease of NADPH absorption after addition of acetyl-CoA to the reaction mixture and therefore the activity. Synthase 1.1 with five deleted amino acids in the linker peptide, showed the 
highest activity of the three synthases. A deletion of ten amino acids in synthase 1.2 led to a reduced activity compared to the two others. 52

Figure 3.9: Dot Blot of the two active synthases 1.0 and 1.1 and the mutated version of synthase 1.1 where the substrate lysine was replaced by a glycine. The membrane was decorated with an acetyllysine antibody followed by an ECL reagent. In the upper section the samples were spotted untreated and the active synthases showed a strong signal in contrast to the mutant. In lower section the samples were incubated with $\mathrm{NAD}^{+}$for 20 min, a substrate for the deacetylation. Upon this $\mathrm{NAD}^{+}$ addition the signals disappeared which demonstrated a deacetylation of the substrate lysines. 54

Figure 3.10: A superimposition of two XICs, obtained by mass spectrometry analysis of the product solution of the enzymatic coupled sirtuin assay. The minor signal represents a mass of 558 dalton and had the same retention time as the ADP-ribose standard. The major signal at a retention time of 38.15 min represents a mass of 600 dalton, which corresponds to the mass of OAADPR. 55

Figure 3.11: Steady-state kinetics of the Hat1 moiety in OAADPRS. Acetylation rates, determined using the enzymatic coupled sirtuin assay, were plotted against the acetyl-CoA concentrations. 56

Figure 3.12: Steady-state kinetics of the CobB moiety in OAADPRS. Deacetylation rates, determined using the enzymatic coupled sirtuin assay, were plotted against $\mathrm{NAD}^{+}$ concentrations. 57

Figure 3.13: Growth test of $E$. coli cells harbouring plasmids with the active synthase 1.1 or the inactive mutant. The control contained an empty plasmid. Cells were induced with $0.5 \mathrm{mM}$ IPTG at timepoint zero. Growth of cells expressing the active synthase was severe delayed after induction while the cells with the inactive synthase grew comparable to the control. 59

Figure 3.14: Western blot of the active synthase expressed from a plasmid with an arabinose inducible promoter. The amount of synthase expressed, utilizing induction levels of 200 and $1000 \mathrm{ppm}$ arabinose were compared. Protein was 
detected with an anti-histidine antibody. Samples were taken before, 2 and $5 \mathrm{~h}$ after induction. On the right purified synthase was blotted as control. At both induction levels protein expression was visible after induction. 60

Figure 3.15: Resazurin assay of cells expressing the active synthase, the inactive mutant and a negative control. Samples were taken at indicated time after induction. The measured fluorescence signals of the formed reagent were normalized to the control, which was set to $100 \%$. In A) cells were induced with 200 ppm arabinose and in B) with $1000 \mathrm{ppm}$ arabinose. A higher expression level of the active synthase and the coupled formation of the metabolite resulted in a higher depression of cell metabolism. 62

Figure 3.16: Determination of $\mathrm{NAD}^{+}$levels in cells expressing the active synthase, the inactive mutant or a negative control. Samples were taken two hours after induction. A formazan reagent was added and the amount of reduction product which depends on NADH concentration in the sample was measured. NAD ${ }^{+}$amounts were calculated from those NADH concentrations. In A) cells were induced with 200 ppm arabinose and in B) with 1000 ppm arabinose. NAD levels were severely decreased when the active synthase was expressed and nearly depleted after increasing the induction to $1000 \mathrm{ppm}$ arabinose. Expression of the inactive synthase resulted in a much lower decrease of $\mathrm{NAD}^{+}$. 64

Figure 3.17: Venn analysis diagram of the gene expression surveys that were performed. Comparing the active synthase to the negative control, 204 genes were regulated. In the samples of the inactive mutant compared to the negativ control 55 genes were regulated. When gene expression was measured in the presence of the active synthase versus the inactive mutant, 74 genes were regulated. Non of the regulated genes were found in all three surveys, only partially in two surveys. 66

Figure 3.18: Reaction scheme of the ß-galactosidase assay. O-nitrophenyl-ß-Dgalactopyranoside (ONPG) is enzymatically converted to galactose and $o$-nitrophenol (ONP) by the lacZ encoded ß-galactosidase. ONP formation can be quantified by measuring its absorption at $420 \mathrm{~nm}$. 70 
Figure 3.19: Scheme representing the lacZ gene for ß-galactosidase and the new promoter region. The original promoter was replaced by the uhpT promoter sequence, including a CAP binding site, a strong-affinity and a low-affinity UhpAbinding region. Depending on synthase and metabolite presence, the promoter should be activated and induce lacZ gene transcription and subsequently ßgalactosidase expression. 70

Figure 3.20: Levels of expressed ß-galactosidase one, two and three hours after induction. All values are depicted in percentage and were normalized to the control, which was set to $100 \%$. Samples harbouring the inactive mutant showed a lower ßgalactosidase expression than the samples with the active synthase. 72

Figure 3.21: Comparison of transcription ratios of genes from Table 3.5, transcribed in the presence of the active synthase (a. s.) or the inactive mutant (i. m.). As reference the housekeeping gene gapA was used and its transcription level was substracted from the values of the genes of interest. nadB was the only gene where, depending on the presence of the active synthase, a significant difference in transcription level could be determined. The transcription of the pnuC gene was increased in the presence of the inactive mutant. 75

Figure 3.22: Reaction scheme for a one-step synthesis of OAADPR. NAD ${ }^{+}$is mixed with sodium carbonate and glacial acetic acid, followed by heating to $90^{\circ} \mathrm{C}$. The product solution containes besides nicotinamide an isomeric mixture of $2^{\prime}$-OAADPR $\left(R_{1}=A c\right.$, $\left.\mathrm{R}_{2}=\mathrm{OH}\right)$ and $3^{\prime}-$ OAADPR $\left(\mathrm{R}_{1}=\mathrm{OH}, \mathrm{R}_{2}=\mathrm{Ac}\right)$. 76

Figure 3.23: Ion exchange chromatogram of the crude reaction products from the onestep synthesis of OAADPR. The chromatography was performed using an aminopropyl-linked silica gel column and $1 \mathrm{M}$ acetic acid as buffer $\mathrm{A}$ and $1 \mathrm{M}$ sodium acetate and $1 \mathrm{M}$ acetic acid as buffer $\mathrm{B}$. The products were eluted over a separation gradient. In the peak at 13.46 min OAADPR was detected by mass spectrometry. 77

Figure 3.24: Chromatogram of the hydrophilic interaction chromatography performed as final purification step in OAADPR synthesis. $10 \mathrm{mM}$ ammonium acetate and acetonitrile were used for the separation gradient on a polyHYDROXYETHYL $A^{\mathrm{TM}}$ 
column. Peaks were collected and analyzed by mass spectrometry for the presence of OAADPR. Mass spectra of the marked retention times $a$ and $b$ are shown in Figure 3.25.

Figure 3.25: Mass spectrum of the sample from the retention time indicated in Figure

3.24. It showes the mass spectrum of OAADPR $(\mathrm{m} / \mathrm{z}=600 \mathrm{Da})$. 79

Figure 3.26: Mass spectrum of the sample from the retention time indicated in Figure 3.24. The main peak at 558 Da represents ADPR. The peaks at $662,744,826$ and 908 Da refer to remaining salt in the sample. 80

Figure 3.27: Western Blots of antibody tests with wild type histone $\mathrm{H} 4$ and differently acylated H4K16 histones. A) Western Blot decorated with anti-histone H4K16 acetyl antibody. Besides the very strong signal for acetylation, also a signal for butyrylation was visible. Crotonylation could not be detected by this antibody. B) Western Blot decorated with anti-crotonyllysine antibody. Crotonylation was recognized best by this antibody and butyrylation to a smaller degree. Wild type histone and acetylation were not recognized by this antibody. 82

Figure 3.28: Western Blots of lysine deacetylation assay samples taken at indicated time points. The assay was performed with CobB deacetylase and histone $\mathrm{H} 4$ carrying different acyl modifications at position K16 as depicted. Signals in the upper sections represent acylated protein, visualized with anti-acetyl or anti-crotonyllysine antibody, respectively. In the lower sections, protein was visualized with anti-H4 antibody for quantification purpose.

Figure 3.29: Western Blots of histone H4K16 debutyrylation by Sirt1. Consumption of the butyryl modification was completed within 4 min after the reaction was initiated. $50 \%$ was cleaved after $1 \mathrm{~min}$.

Figure 3.30: Western Blots of lysine deacetylation on the left and decrotonylation on the right by Sirt1. Measurements of the individual modifications are not consistent and therefore regarded as preliminary results. Cleavage of acetyl groups off histone H4K16 took either about 30 min or longer. Rates of decrotonylation were between $2 \mathrm{~min}$ and more than $30 \mathrm{~min}$. 85 
Figure 4.1: Scheme of different Iysine modifications used as substrates in the deacylation assays. 


\subsection{Abbreviations}

AARS

$\mathrm{ADH}$

ADP

ADPR

APS

ARH3

ATP

CAP

CIP

CoA

$\mathrm{CP}$

DTT

$\mathrm{ECL}$

FADH

$\mathrm{FMNH}$

FDR

GAPDH

GFP

Glu6P

Hat1

Hda1

HDAC

Hst2

IPTG

LB

Lys
aminoacyl-tRNA synthetase

alcohol dehydrogenase

adenosine diphosphate

adenosine diphosphate ribose

ammonium persulfate

ADP-ribosylhydrolase 3

adenosine triphosphate

catabolite gene activator protein

calf intestinal alkaline phosphate

coenzyme A

crossing point

dithiothreitol

enhanced chemiluminescence

flavinadenine dinucleotide (semiquinone)

flavin mononucleotide (semiquinone)

false discovery rate

glyceraldehyde-3-phosphate dehydrogenase

green fluorescent porotein

glucose 6-phosphate

histone acetyltranferase 1

histone deacetylase 1

histone deacetylase

homologue of Sir two 2

isopropyl ß-D-1 thiogalactopyranuside

lysogeny broth

lysine 
MBP

$N A D^{+}$

$\mathrm{NADH}$

$\mathrm{NADPH}$

NAM

Ni-NTA

NES

NUDIX

OAADPR

OAADPRS

ONP

ONPG

PAGE

PCR

PGK

PIC

PMSF

PRPP

PTM

PTS

qRT-PCR

Rpd3

ROS

RT

SDS

SIR

TAL

TCA

TEMED maltose binding protein

nicotinamide adenine dinucleotide

nicotinamide adenine dinucleotide hydride

nicotinamide adenine dinucleotide phosphate

nicotinamide

nickel-nitrilotriacetic acid

nuclear export signal

nucleoside diphosphate linked to another moiety $x$

O-acetyl-ADP-ribose

$O$-acetyl-ADP-ribose synthase

o-nitrophenol

o-nitrophenyl-ß-D-galactopyranoside

polyacrylamide gel electrophoresis

polymerase chain reaction

phosphoglycerate kinase

protease inhibitor cocktail

phenylmethylsulfonyl fluoride

5-phospho- $\alpha$-D-ribose 1-diphosphate

posttranslational modification

PEP - carbohydrate phosphotranferase system

quantitative real-time-polymerase chain reaction

reduced potassium dependency 3

reactive oxygen species

room temperature

sodium dodecylsulfate

silent mating type information regulator

transcriptome-analysis laboratory

tricarboxylic acid

tetramethylethylenediamine 
TEV

TLC

TRPM2

UAA

XIC tobacco etch virus

thin layer chromatography

transient receptor potential malastatin-related channel 2

unnatural amino acid

extracted ion chromatogram 


\subsection{Supplemetry data}

\subsubsection{Gene expression analysis}

Complete datasets of the three surveys are depicted in tables 7.1-7.3. A positive fold change indicates an upregulation of a gene in the presence of the first construct named in the legend of the table compared to the presence of the second construct. A negative value indicates a downregulation of the gene.

Table 7.1: Survey with the active synthase versus the inactive mutant.

\begin{tabular}{lcllllll}
\hline Symbol & $\begin{array}{c}\text { fold } \\
\text { change }\end{array}$ & p-value & FDR & Symbol & $\begin{array}{c}\text { fold } \\
\text { change }\end{array}$ & p-value & FDR \\
\hline uhpT & 16,68 & $3,57 \mathrm{E}-10$ & $0,00 \%$ & gark & 2,40 & $4,27 \mathrm{E}-08$ & $0,00 \%$ \\
uhpT & 16,68 & $1,91 \mathrm{E}-12$ & $0,00 \%$ & fruB & 2,40 & $2,12 \mathrm{E}-06$ & $0,01 \%$ \\
ECs0778 & 14,85 & $3,51 \mathrm{E}-12$ & $0,00 \%$ & IfhA & 2,39 & $7,08 \mathrm{E}-03$ & $2,22 \%$ \\
nadA & 14,08 & $1,63 \mathrm{E}-12$ & $0,00 \%$ & fruK & 2,33 & $2,81 \mathrm{E}-07$ & $0,00 \%$ \\
nadA & 13,61 & $2,83 \mathrm{E}-12$ & $0,00 \%$ & C-5225 & 2,28 & $7,06 \mathrm{E}-09$ & $0,00 \%$ \\
nadA & 12,80 & $2,78 \mathrm{E}-11$ & $0,00 \%$ & araB & 2,24 & $4,75 \mathrm{E}-07$ & $0,01 \%$ \\
nadB & 10,18 & $2,26 \mathrm{E}-12$ & $0,00 \%$ & rhaA & 2,23 & $1,57 \mathrm{E}-06$ & $0,01 \%$ \\
yjiY & 5,22 & $2,02 \mathrm{E}-10$ & $0,00 \%$ & phoH & 2,15 & $7,21 \mathrm{E}-08$ & $0,00 \%$ \\
yjiY & 4,93 & $8,46 \mathrm{E}-11$ & $0,00 \%$ & b2885 & 2,12 & $6,79 \mathrm{E}-08$ & $0,00 \%$ \\
pnuC & 4,33 & $4,17 \mathrm{E}-10$ & $0,00 \%$ & ECs4830 & 2,10 & $1,37 \mathrm{E}-08$ & $0,00 \%$ \\
garL & 3,94 & $1,26 \mathrm{E}-08$ & $0,00 \%$ & ygfM & 2,05 & $4,04 \mathrm{E}-06$ & $0,02 \%$ \\
garR & 3,79 & $2,51 \mathrm{E}-08$ & $0,00 \%$ & ilvC & 2,04 & $1,48 \mathrm{E}-07$ & $0,00 \%$ \\
araD & 3,27 & $2,10 \mathrm{E}-09$ & $0,00 \%$ & Z3609 & 2,03 & $1,04 \mathrm{E}-02$ & $2,94 \%$ \\
b2999 & 2,75 & $5,77 \mathrm{E}-09$ & $0,00 \%$ & xdhD & 2,03 & $1,50 \mathrm{E}-06$ & $0,01 \%$ \\
araA & 2,71 & $3,06 \mathrm{E}-09$ & $0,00 \%$ & ECs4006 & 2,02 & $5,87 \mathrm{E}-07$ & $0,01 \%$ \\
c_4110 & 2,58 & $1,06 \mathrm{E}-04$ & $0,12 \%$ & fadB & 2,01 & $1,21 \mathrm{E}-07$ & $0,00 \%$ \\
soxS & 2,47 & $7,31 \mathrm{E}-09$ & $0,00 \%$ & lacY & 2,00 & $4,31 \mathrm{E}-08$ & $0,00 \%$ \\
\hline
\end{tabular}




\begin{tabular}{lcllllll}
\hline Symbol & $\begin{array}{c}\text { fold } \\
\text { change }\end{array}$ & p-value & FDR & Symbol & $\begin{array}{c}\text { fold } \\
\text { change }\end{array}$ & p-value & FDR \\
\hline ECs3221 & $-2,03$ & $1,38 E-04$ & $0,14 \%$ & Z4171 & $-2,45$ & $6,34 E-04$ & $0,40 \%$ \\
NinE & $-2,04$ & $4,52 E-05$ & $0,07 \%$ & typA & $-2,47$ & $8,29 E-09$ & $0,00 \%$ \\
ECs0299 & $-2,06$ & $1,43 E-04$ & $0,15 \%$ & nupC & $-2,49$ & $8,65 E-09$ & $0,00 \%$ \\
Z0336 & $-2,07$ & $2,76 E-05$ & $0,05 \%$ & ymfD & $-2,52$ & $4,01 E-05$ & $0,06 \%$ \\
speA & $-2,08$ & $1,07 E-06$ & $0,01 \%$ & Z2123 & $-2,52$ & $2,67 E-03$ & $1,10 \%$ \\
yehM & $-2,11$ & $2,54 E-04$ & $0,22 \%$ & ECs1219 & $-2,57$ & $4,63 E-05$ & $0,07 \%$ \\
lysA & $-2,16$ & $5,26 E-09$ & $0,00 \%$ & ECs1333 & $-2,58$ & $4,07 E-06$ & $0,02 \%$ \\
ECs1544 & $-2,20$ & $9,46 E-03$ & $2,75 \%$ & Z2119 & $-2,63$ & $5,91 E-04$ & $0,38 \%$ \\
ECs3707 & $-2,20$ & $6,61 E-05$ & $0,09 \%$ & gpmA & $-2,71$ & $1,15 E-08$ & $0,00 \%$ \\
C_1234 & $-2,21$ & $1,79 E-04$ & $0,17 \%$ & C_0036 & $-2,79$ & $6,00 E-03$ & $1,96 \%$ \\
glyS & $-2,25$ & $4,18 E-06$ & $0,02 \%$ & c_5185 & $-2,82$ & $1,29 E-02$ & $3,46 \%$ \\
ECs3483 & $-2,26$ & $9,52 E-04$ & $0,53 \%$ & yifE & $-2,96$ & $2,34 E-09$ & $0,00 \%$ \\
aceA & $-2,26$ & $2,08 E-08$ & $0,00 \%$ & c_2556 & $-2,97$ & $7,45 E-10$ & $0,00 \%$ \\
glgP & $-2,27$ & $2,25 E-07$ & $0,00 \%$ & gnd & $-3,02$ & $5,22 E-10$ & $0,00 \%$ \\
L7055 & $-2,27$ & $1,84 E-03$ & $0,84 \%$ & prs & $-3,05$ & $1,02 E-09$ & $0,00 \%$ \\
ECs1944 & $-2,29$ & $1,91 E-05$ & $0,04 \%$ & ompA & $-3,93$ & $2,19 E-08$ & $0,00 \%$ \\
aceB & $-2,30$ & $5,80 E-07$ & $0,01 \%$ & c_2455 & $-4,31$ & $1,09 E-04$ & $0,12 \%$ \\
aceA & $-2,34$ & $6,18 E-07$ & $0,01 \%$ & Z3204 & $-6,80$ & $8,10 E-08$ & $0,00 \%$ \\
hmp & $-2,35$ & $2,88 E-08$ & $0,00 \%$ & ECs2845 & $-6,89$ & $7,59 E-08$ & $0,00 \%$ \\
C_5405 & $-2,36$ & $2,32 E-05$ & $0,05 \%$ & Z4171 & $-2,45$ & $6,34 E-04$ & $0,40 \%$ \\
Z1155 & $-2,44$ & $1,16 E-05$ & $0,03 \%$ & typA & $-2,47$ & $8,29 E-09$ & $0,00 \%$ \\
\hline & & & & & & & \\
\hline
\end{tabular}


Table 7.2: Survey with the active synthase versus the control.

\begin{tabular}{|c|c|c|c|c|c|c|c|}
\hline Symbol & $\begin{array}{c}\text { fold } \\
\text { change }\end{array}$ & p-value & FDR & Symbol & $\begin{array}{c}\text { fold } \\
\text { change }\end{array}$ & p-value & FDR \\
\hline cobB & 1071,41 & $1,10 \mathrm{E}-14$ & $0,00 \%$ & fruk & 2,50 & $3,49 \mathrm{E}-07$ & $0,00 \%$ \\
\hline ECs1498 & 852,03 & $3,40 \mathrm{E}-12$ & $0,00 \%$ & nth & 2,40 & $3,54 \mathrm{E}-05$ & $0,04 \%$ \\
\hline cobB & 793,97 & $2,58 \mathrm{E}-14$ & $0,00 \%$ & tomB & 2,35 & $1,76 \mathrm{E}-08$ & $0,00 \%$ \\
\hline uhpt & 25,00 & $2,98 \mathrm{E}-10$ & $0,00 \%$ & phoE & 2,34 & $7,90 \mathrm{E}-06$ & $0,01 \%$ \\
\hline uhpT & 20,67 & $2,58 \mathrm{E}-12$ & $0,00 \%$ & araF & 2,34 & 4,19E-07 & $0,00 \%$ \\
\hline $\operatorname{nad} A$ & 17,70 & 2,61E-11 & $0,00 \%$ & c_3614 & 2,32 & $5,24 \mathrm{E}-06$ & $0,01 \%$ \\
\hline ECs0778 & 17,63 & $5,21 \mathrm{E}-12$ & $0,00 \%$ & yggA & 2,29 & $2,18 \mathrm{E}-02$ & $4,82 \%$ \\
\hline $\operatorname{nad} A$ & 16,57 & $3,84 \mathrm{E}-12$ & $0,00 \%$ & $x d h D$ & 2,29 & $1,05 \mathrm{E}-06$ & $0,00 \%$ \\
\hline $\operatorname{nad} A$ & 15,11 & $3,19 \mathrm{E}-12$ & $0,00 \%$ & ECs1634 & 2,29 & $4,52 \mathrm{E}-07$ & $0,00 \%$ \\
\hline nadB & 12,86 & $2,55 \mathrm{E}-12$ & $0,00 \%$ & fadB & 2,29 & $7,89 \mathrm{E}-08$ & $0,00 \%$ \\
\hline pnuC & 5,65 & $2,70 \mathrm{E}-10$ & $0,00 \%$ & garL & 2,28 & 1,51E-06 & $0,00 \%$ \\
\hline $\operatorname{araA}$ & 4,88 & $1,91 \mathrm{E}-10$ & $0,00 \%$ & lolc & 2,26 & $1,52 \mathrm{E}-06$ & $0,00 \%$ \\
\hline araD & 4,85 & $5,27 \mathrm{E}-10$ & $0,00 \%$ & cysN & 2,25 & $3,62 \mathrm{E}-08$ & $0,00 \%$ \\
\hline araB & 3,70 & $2,68 \mathrm{E}-08$ & $0,00 \%$ & can & 2,23 & $5,82 \mathrm{E}-08$ & $0,00 \%$ \\
\hline yjiY & 3,52 & 4,13E-09 & $0,00 \%$ & c_1549 & 2,22 & $5,53 \mathrm{E}-06$ & $0,01 \%$ \\
\hline yjiY & 3,39 & 1,66E-09 & $0,00 \%$ & ECs1176 & 2,22 & 9,37E-08 & $0,00 \%$ \\
\hline cysJ & 3,15 & $1,60 \mathrm{E}-06$ & $0,00 \%$ & z3357 & 2,22 & $1,63 \mathrm{E}-07$ & $0,00 \%$ \\
\hline ECs2987 & 2,99 & $1,81 \mathrm{E}-05$ & $0,02 \%$ & garR & 2,21 & $3,14 \mathrm{E}-06$ & $0,01 \%$ \\
\hline cysJ & 2,84 & $1,68 \mathrm{E}-07$ & $0,00 \%$ & усім & 2,20 & $3,34 \mathrm{E}-07$ & $0,00 \%$ \\
\hline hha & 2,69 & $2,14 \mathrm{E}-08$ & $0,00 \%$ & cysP & 2,18 & $8,41 \mathrm{E}-08$ & $0,00 \%$ \\
\hline b2999 & 2,65 & $1,82 \mathrm{E}-08$ & $0,00 \%$ & ygeX & 2,18 & $2,82 \mathrm{E}-08$ & $0,00 \%$ \\
\hline Z3356 & 2,64 & 7,87E-05 & $0,06 \%$ & ECs3745 & 2,17 & $5,00 \mathrm{E}-08$ & $0,00 \%$ \\
\hline c_2248 & 2,61 & 2,07E-07 & $0,00 \%$ & c_1538 & 2,16 & $1,01 \mathrm{E}-07$ & $0,00 \%$ \\
\hline fruB & 2,60 & $2,48 \mathrm{E}-06$ & $0,01 \%$ & yebo & 2,15 & $2,15 \mathrm{E}-07$ & $0,00 \%$ \\
\hline c_1268 & 2,58 & 4,76E-06 & $0,01 \%$ & ECs2988 & 2,14 & $2,90 \mathrm{E}-06$ & $0,01 \%$ \\
\hline c_3169 & 2,57 & 4,90E-07 & $0,00 \%$ & ECs1625 & 2,14 & $9,83 \mathrm{E}-08$ & $0,00 \%$ \\
\hline
\end{tabular}




\begin{tabular}{|c|c|c|c|c|c|c|c|}
\hline Symbol & $\begin{array}{c}\text { fold } \\
\text { change }\end{array}$ & p-value & FDR & Symbol & $\begin{array}{c}\text { fold } \\
\text { change }\end{array}$ & p-value & FDR \\
\hline z1887 & 2,14 & $1,18 \mathrm{E}-07$ & $0,00 \%$ & ECs1187 & 2,02 & $8,28 \mathrm{E}-07$ & $0,00 \%$ \\
\hline cysN & 2,13 & $7,90 \mathrm{E}-07$ & $0,00 \%$ & yeiA & 2,01 & $2,80 \mathrm{E}-07$ & $0,00 \%$ \\
\hline b1899 & 2,12 & $3,41 \mathrm{E}-08$ & $0,00 \%$ & $y b c U$ & 2,01 & 8,59E-06 & $0,01 \%$ \\
\hline ygeY & 2,11 & $1,49 \mathrm{E}-07$ & $0,00 \%$ & c_4113 & 2,01 & 1,99E-07 & $0,00 \%$ \\
\hline $\mathrm{fadB}$ & 2,11 & $2,62 \mathrm{E}-05$ & $0,03 \%$ & ycfU & 2,01 & 5,92E-07 & $0,00 \%$ \\
\hline pstA & 2,10 & 4,89E-08 & $0,00 \%$ & b2858 & 2,01 & $2,84 \mathrm{E}-07$ & $0,00 \%$ \\
\hline soxS & 2,10 & $7,90 \mathrm{E}-08$ & $0,00 \%$ & Z1889 & 2,01 & $6,45 \mathrm{E}-07$ & $0,00 \%$ \\
\hline ECs1250 & 2,10 & 1,95E-03 & $0,71 \%$ & yrdD & 2,01 & $3,04 \mathrm{E}-07$ & $0,00 \%$ \\
\hline phoH & 2,10 & 2,19E-07 & $0,00 \%$ & c_2555 & 2,01 & 6,86E-06 & $0,01 \%$ \\
\hline ECs1636 & 2,09 & $2,85 \mathrm{E}-07$ & $0,00 \%$ & Z0311 & 2,00 & $4,42 E-06$ & $0,01 \%$ \\
\hline b1898 & 2,08 & 1,14E-06 & $0,00 \%$ & Z1449 & 2,00 & $8,37 \mathrm{E}-06$ & $0,01 \%$ \\
\hline ilvC & 2,08 & 2,83E-07 & $0,00 \%$ & nirD & $-2,00$ & $1,43 \mathrm{E}-07$ & $0,00 \%$ \\
\hline cysC & 2,07 & $5,85 \mathrm{E}-07$ & $0,00 \%$ & gltK & $-2,01$ & 8,39E-07 & $0,00 \%$ \\
\hline z0970 & 2,07 & $4,02 E-06$ & $0,01 \%$ & Z2229 & $-2,02$ & 5,96E-07 & $0,00 \%$ \\
\hline hyuA & 2,06 & 1,17E-06 & $0,00 \%$ & Z0388 & $-2,02$ & $8,45 \mathrm{E}-05$ & $0,07 \%$ \\
\hline ECs1294 & 2,05 & $1,18 \mathrm{E}-04$ & $0,08 \%$ & $\mathrm{bdm}$ & $-2,02$ & 4,44E-07 & $0,00 \%$ \\
\hline ykgK & 2,05 & 9,38E-06 & $0,01 \%$ & $\operatorname{iad} A$ & $-2,03$ & 4,85E-08 & $0,00 \%$ \\
\hline ykgG & 2,05 & $1,29 \mathrm{E}-07$ & $0,00 \%$ & gltL & $-2,04$ & $2,32 \mathrm{E}-07$ & $0,00 \%$ \\
\hline c_2858 & 2,05 & $1,26 \mathrm{E}-03$ & $0,51 \%$ & yjiG & $-2,04$ & $9,06 \mathrm{E}-07$ & $0,00 \%$ \\
\hline cysN & 2,04 & $6,48 \mathrm{E}-06$ & $0,01 \%$ & ribA & $-2,04$ & 9,95E-06 & $0,02 \%$ \\
\hline c_3167 & 2,04 & $2,18 \mathrm{E}-07$ & $0,00 \%$ & $y k f H$ & $-2,04$ & $6,71 \mathrm{E}-03$ & $1,89 \%$ \\
\hline fadB & 2,03 & $4,16 \mathrm{E}-05$ & $0,04 \%$ & rpmE & $-2,04$ & $2,73 E-08$ & $0,00 \%$ \\
\hline ECs1217 & 2,03 & $9,78 \mathrm{E}-08$ & $0,00 \%$ & yajo & $-2,05$ & 2,27E-05 & $0,03 \%$ \\
\hline cysN & 2,02 & $3,68 \mathrm{E}-07$ & $0,00 \%$ & intB & $-2,05$ & 3,74E-07 & $0,00 \%$ \\
\hline celF & 2,02 & $1,45 \mathrm{E}-06$ & $0,00 \%$ & fdoH & $-2,05$ & 4,11E-05 & $0,04 \%$ \\
\hline ygeY & 2,02 & $1,47 \mathrm{E}-07$ & $0,00 \%$ & ydjE & $-2,06$ & $3,18 \mathrm{E}-04$ & $0,18 \%$ \\
\hline
\end{tabular}




\begin{tabular}{|c|c|c|c|c|c|c|c|}
\hline Symbol & $\begin{array}{c}\text { fold } \\
\text { change }\end{array}$ & p-value & FDR & Symbol & $\begin{array}{c}\text { fold } \\
\text { change }\end{array}$ & p-value & FDR \\
\hline ribA & $-2,06$ & $5,13 E-06$ & $0,01 \%$ & ECs0473 & $-2,20$ & $1,38 \mathrm{E}-06$ & $0,00 \%$ \\
\hline evgs & $-2,06$ & 3,31E-07 & $0,00 \%$ & sers & $-2,22$ & $1,47 E-08$ & $0,00 \%$ \\
\hline osmE & $-2,07$ & $5,69 E-06$ & $0,01 \%$ & yicR & $-2,23$ & $4,73 E-07$ & $0,00 \%$ \\
\hline ECs1156 & $-2,08$ & $6,85 \mathrm{E}-05$ & $0,06 \%$ & ECs1544 & $-2,23$ & $1,48 \mathrm{E}-02$ & $3,52 \%$ \\
\hline ECs0299 & $-2,08$ & $2,71 \mathrm{E}-04$ & $0,16 \%$ & raiA & $-2,23$ & $7,86 \mathrm{E}-07$ & $0,00 \%$ \\
\hline ompC & $-2,08$ & 1,47E-07 & $0,00 \%$ & yfbT & $-2,24$ & $2,18 \mathrm{E}-07$ & $0,00 \%$ \\
\hline ECs2038 & $-2,10$ & $1,63 \mathrm{E}-05$ & $0,02 \%$ & ychH & $-2,24$ & $1,04 \mathrm{E}-07$ & $0,00 \%$ \\
\hline $\mathrm{racC}$ & $-2,10$ & $4,73 \mathrm{E}-04$ & $0,24 \%$ & $\mathrm{dppC}$ & $-2,25$ & $4,98 \mathrm{E}-08$ & $0,00 \%$ \\
\hline ECs3622 & $-2,11$ & $3,70 \mathrm{E}-04$ & $0,20 \%$ & Z4982 & $-2,28$ & $2,25 \mathrm{E}-08$ & $0,00 \%$ \\
\hline $\operatorname{ygdR}$ & $-2,11$ & $7,65 \mathrm{E}-07$ & $0,00 \%$ & c_4992 & $-2,28$ & 2,47E-06 & $0,01 \%$ \\
\hline ECs3249 & $-2,12$ & $8,29 E-08$ & $0,00 \%$ & psuk & $-2,29$ & $1,27 \mathrm{E}-06$ & $0,00 \%$ \\
\hline ECs3771 & $-2,13$ & $4,25 E-07$ & $0,00 \%$ & c_1234 & $-2,30$ & $2,73 \mathrm{E}-04$ & $0,16 \%$ \\
\hline Z1419 & $-2,13$ & $2,46 \mathrm{E}-04$ & $0,15 \%$ & ECs1219 & $-2,30$ & $2,39 \mathrm{E}-04$ & $0,14 \%$ \\
\hline yjbJ & $-2,13$ & $2,43 E-06$ & $0,01 \%$ & - & $-2,31$ & $9,43 E-08$ & $0,00 \%$ \\
\hline $\operatorname{csp} A$ & $-2,14$ & 1,10E-07 & $0,00 \%$ & uspG & $-2,32$ & 8,97E-09 & $0,00 \%$ \\
\hline c_5381 & $-2,14$ & $6,89 E-05$ & $0,06 \%$ & c_0438 & $-2,33$ & $6,24 \mathrm{E}-08$ & $0,00 \%$ \\
\hline ycgF & $-2,14$ & $8,24 \mathrm{E}-06$ & $0,01 \%$ & $\operatorname{iad} A$ & $-2,35$ & $4,89 E-08$ & $0,00 \%$ \\
\hline yehM & $-2,14$ & $4,80 \mathrm{E}-04$ & $0,24 \%$ & ECs2924 & $-2,36$ & $7,44 \mathrm{E}-07$ & $0,00 \%$ \\
\hline ECs4422 & $-2,14$ & $1,10 \mathrm{E}-07$ & $0,00 \%$ & Z2286 & $-2,37$ & $2,79 E-06$ & $0,01 \%$ \\
\hline melA & $-2,15$ & $6,59 \mathrm{E}-05$ & $0,06 \%$ & $\mathrm{ECs} 0348$ & $-2,38$ & $3,92 \mathrm{E}-08$ & $0,00 \%$ \\
\hline appB & $-2,16$ & $1,23 \mathrm{E}-07$ & $0,00 \%$ & speA & $-2,38$ & $5,30 \mathrm{E}-07$ & $0,00 \%$ \\
\hline yihD & $-2,16$ & $2,66 \mathrm{E}-07$ & $0,00 \%$ & $\mathrm{pflB}$ & $-2,39$ & $4,21 E-07$ & $0,00 \%$ \\
\hline slp & $-2,18$ & $1,38 \mathrm{E}-06$ & $0,00 \%$ & Z4855 & $-2,39$ & $1,64 \mathrm{E}-04$ & $0,11 \%$ \\
\hline Z3916 & $-2,18$ & $2,16 \mathrm{E}-03$ & $0,76 \%$ & ygdH & $-2,40$ & $3,04 \mathrm{E}-07$ & $0,00 \%$ \\
\hline essQ & $-2,18$ & $2,03 \mathrm{E}-03$ & $0,73 \%$ & molR_D & $-2,41$ & $8,85 \mathrm{E}-08$ & $0,00 \%$ \\
\hline ompF & $-2,20$ & 2,83E-07 & $0,00 \%$ & ydit & $-2,41$ & $1,48 \mathrm{E}-05$ & $0,02 \%$ \\
\hline
\end{tabular}




\begin{tabular}{|c|c|c|c|c|c|c|c|}
\hline Symbol & $\begin{array}{c}\text { fold } \\
\text { change }\end{array}$ & p-value & FDR & Symbol & $\begin{array}{c}\text { fold } \\
\text { change }\end{array}$ & p-value & FDR \\
\hline ECs2085 & $-2,42$ & $3,73 \mathrm{E}-06$ & $0,01 \%$ & narl & $-3,01$ & $8,56 \mathrm{E}-03$ & $2,28 \%$ \\
\hline gapA & $-2,43$ & $2,87 \mathrm{E}-08$ & $0,00 \%$ & cheZ & $-3,07$ & 1,33E-03 & $0,53 \%$ \\
\hline grxB & $-2,44$ & $4,34 \mathrm{E}-07$ & $0,00 \%$ & rne & $-3,09$ & $2,01 \mathrm{E}-08$ & $0,00 \%$ \\
\hline Ipp & $-2,45$ & $2,16 \mathrm{E}-08$ & $0,00 \%$ & c_1176 & $-3,12$ & 7,98E-07 & $0,00 \%$ \\
\hline djlB & $-2,46$ & $8,24 \mathrm{E}-05$ & $0,07 \%$ & gnd & $-3,16$ & 1,17E-09 & $0,00 \%$ \\
\hline Z4077 & $-2,47$ & 4,39E-05 & $0,04 \%$ & Z2119 & $-3,22$ & 3,51E-04 & $0,19 \%$ \\
\hline $\operatorname{csp} E$ & $-2,47$ & $2,08 \mathrm{E}-08$ & $0,00 \%$ & gnd & $-3,28$ & $7,07 \mathrm{E}-10$ & $0,00 \%$ \\
\hline hmpA & $-2,48$ & $1,70 \mathrm{E}-07$ & $0,00 \%$ & ydaG & $-3,33$ & $3,86 \mathrm{E}-08$ & $0,00 \%$ \\
\hline ECs3104 & $-2,55$ & $1,08 \mathrm{E}-07$ & $0,00 \%$ & $\mathrm{hmp}$ & $-3,54$ & 3,28E-09 & $0,00 \%$ \\
\hline $\operatorname{csp} E$ & $-2,56$ & $7,40 \mathrm{E}-08$ & $0,00 \%$ & aceB & $-3,68$ & 4,36E-08 & $0,00 \%$ \\
\hline yifE & $-2,59$ & $1,55 \mathrm{E}-08$ & $0,00 \%$ & aceA & $-3,75$ & 4,83E-08 & $0,00 \%$ \\
\hline nupC & $-2,62$ & $1,34 \mathrm{E}-08$ & $0,00 \%$ & aceA & $-3,80$ & 1,06E-09 & $0,00 \%$ \\
\hline ECs3483 & $-2,64$ & $6,29 \mathrm{E}-04$ & $0,29 \%$ & gpmA & $-4,07$ & 1,89E-09 & $0,00 \%$ \\
\hline NinE & $-2,66$ & $1,05 \mathrm{E}-05$ & $0,02 \%$ & ompA & $-4,20$ & $3,60 \mathrm{E}-08$ & $0,00 \%$ \\
\hline b0501 & $-2,66$ & $5,06 \mathrm{E}-07$ & $0,00 \%$ & c_0036 & $-4,22$ & 1,66E-03 & $0,63 \%$ \\
\hline glgP & $-2,69$ & $1,23 \mathrm{E}-07$ & $0,00 \%$ & prs & $-4,27$ & $3,05 \mathrm{E}-10$ & $0,00 \%$ \\
\hline ybeJ & $-2,70$ & 1,87E-08 & $0,00 \%$ & ymfD & $-4,81$ & 1,84E-06 & $0,01 \%$ \\
\hline yjiD & $-2,77$ & $1,49 \mathrm{E}-05$ & $0,02 \%$ & papD & $-5,06$ & $2,13 \mathrm{E}-03$ & $0,75 \%$ \\
\hline ECs0694 & $-2,78$ & $4,75 E-09$ & $0,00 \%$ & c_2455 & $-5,15$ & 1,07E-04 & $0,08 \%$ \\
\hline c_0042 & $-2,81$ & $6,17 \mathrm{E}-07$ & $0,00 \%$ & c_3561 & $-8,81$ & $9,09 \mathrm{E}-03$ & $2,40 \%$ \\
\hline Z0039 & $-2,89$ & $4,80 \mathrm{E}-08$ & $0,00 \%$ & $w b d N$ & $-11,74$ & $2,76 \mathrm{E}-08$ & $0,00 \%$ \\
\hline typA & $-2,89$ & 5,69E-09 & $0,00 \%$ & ECs2845 & $-12,92$ & $2,03 E-08$ & $0,00 \%$ \\
\hline speA & $-2,90$ & $1,48 \mathrm{E}-07$ & $0,00 \%$ & & & & \\
\hline melB & $-2,91$ & $4,38 \mathrm{E}-08$ & $0,00 \%$ & & & & \\
\hline$g|t|$ & $-2,93$ & 4,07E-08 & $0,00 \%$ & & & & \\
\hline glys & $-2,93$ & 1,14E-06 & $0,00 \%$ & & & & \\
\hline
\end{tabular}


Table 7.3: Survey with the inactive mutant versus the control.

\begin{tabular}{|c|c|c|c|c|c|c|c|}
\hline Symbol & $\begin{array}{c}\text { fold } \\
\text { change }\end{array}$ & $p$-value & FDR & Symbol & $\begin{array}{c}\text { fold } \\
\text { change }\end{array}$ & p-value & FDR \\
\hline cobB & 1071,41 & $1,10 \mathrm{E}-14$ & $0,00 \%$ & ECs1637 & 2,07 & 4,64E-06 & $0,02 \%$ \\
\hline cobB & 455,53 & $5,11 \mathrm{E}-14$ & $0,00 \%$ & ECs1217 & 2,06 & 8,50E-08 & $0,00 \%$ \\
\hline ECs1498 & 440,73 & $7,64 \mathrm{E}-12$ & $0,00 \%$ & ECs1625 & 2,06 & 1,46E-07 & $0,00 \%$ \\
\hline ECs1805 & 4,23 & $1,95 \mathrm{E}-02$ & $4,93 \%$ & borW & 2,05 & $5,10 \mathrm{E}-07$ & $0,01 \%$ \\
\hline Z0972 & 2,81 & $6,62 \mathrm{E}-04$ & $0,44 \%$ & ECs2162 & 2,05 & 8,11E-07 & $0,01 \%$ \\
\hline Z0970 & 2,75 & $3,27 \mathrm{E}-07$ & $0,01 \%$ & cysJ & 2,02 & 5,86E-05 & $0,10 \%$ \\
\hline ECs1250 & 2,48 & $5,64 \mathrm{E}-04$ & $0,40 \%$ & ECs1634 & 2,01 & 1,62E-06 & $0,01 \%$ \\
\hline ECs0834 & 2,39 & $3,29 \mathrm{E}-06$ & $0,02 \%$ & ylaC & $-2,01$ & 1,24E-05 & $0,04 \%$ \\
\hline ECs3794 & 2,38 & $3,41 \mathrm{E}-03$ & $1,38 \%$ & c_4992 & $-2,02$ & 8,19E-06 & $0,03 \%$ \\
\hline ECs1187 & 2,38 & $1,64 \mathrm{E}-07$ & $0,01 \%$ & $\mathrm{dppC}$ & $-2,08$ & 1,06E-07 & $0,00 \%$ \\
\hline Z2346 & 2,28 & 8,84E-03 & $2,74 \%$ & b0501 & $-2,10$ & $4,05 \mathrm{E}-06$ & $0,02 \%$ \\
\hline ECs1836 & 2,26 & $1,98 \mathrm{E}-07$ & $0,01 \%$ & ECs0348 & $-2,13$ & 1,11E-07 & $0,00 \%$ \\
\hline c_1580 & 2,26 & 5,99E-08 & $0,00 \%$ & Z0388 & $-2,14$ & 4,91E-05 & $0,09 \%$ \\
\hline ECs2987 & 2,23 & $1,64 \mathrm{E}-04$ & $0,18 \%$ & Z0655 & $-2,14$ & 3,68E-06 & $0,02 \%$ \\
\hline ECs0832 & 2,22 & $1,92 \mathrm{E}-05$ & $0,05 \%$ & c_4110 & $-2,20$ & 7,53E-04 & $0,48 \%$ \\
\hline $\mathrm{rfbA}$ & 2,22 & $2,79 E-04$ & $0,25 \%$ & hokA & $-2,26$ & 1,91E-08 & $0,00 \%$ \\
\hline ECs2988 & 2,20 & $2,24 \mathrm{E}-06$ & $0,02 \%$ & ECs1254 & $-2,29$ & 7,71E-03 & $2,48 \%$ \\
\hline ykgG & 2,19 & $2,38 \mathrm{E}-07$ & $0,01 \%$ & melB & $-2,36$ & $2,33 \mathrm{E}-07$ & $0,01 \%$ \\
\hline Z3079 & 2,19 & $8,04 \mathrm{E}-05$ & $0,11 \%$ & c_0471 & $-2,41$ & 1,23E-05 & $0,04 \%$ \\
\hline c_1548 & 2,17 & $2,79 \mathrm{E}-08$ & $0,00 \%$ & Z4982 & $-2,49$ & 9,86E-09 & $0,00 \%$ \\
\hline Z1449 & 2,16 & 3,87E-06 & $0,02 \%$ & & $-2,52$ & $4,40 \mathrm{E}-08$ & $0,00 \%$ \\
\hline c_1549 & 2,14 & $8,00 \mathrm{E}-06$ & $0,03 \%$ & c_0042 & $-2,59$ & 1,14E-06 & $0,01 \%$ \\
\hline Z3356 & 2,11 & $4,56 \mathrm{E}-04$ & $0,35 \%$ & Z3651 & $-2,61$ & 4,27E-03 & $1,61 \%$ \\
\hline c_3169 & 2,10 & $3,00 \mathrm{E}-06$ & $0,02 \%$ & ydaG & $-2,63$ & $2,13 \mathrm{E}-07$ & $0,01 \%$ \\
\hline nth & 2,10 & $1,12 \mathrm{E}-04$ & $0,14 \%$ & c_5225 & $-2,71$ & 3,79E-09 & $0,00 \%$ \\
\hline Z3357 & 2,08 & $3,14 \mathrm{E}-07$ & $0,01 \%$ & nirD & $-2,77$ & 7,35E-09 & $0,00 \%$ \\
\hline
\end{tabular}




\begin{tabular}{lccccccc}
\hline Symbol & $\begin{array}{c}\text { fold } \\
\text { change }\end{array}$ & p-value & FDR & Symbol & $\begin{array}{c}\text { fold } \\
\text { change }\end{array}$ & p-value & FDR \\
\hline Z0039 & $-2,93$ & $4,29 E-08$ & $0,00 \%$ & molR_D & $-3,23$ & $9,44 \mathrm{E}-09$ & $0,00 \%$ \\
ECs2924 & $-3,04$ & $1,04 \mathrm{E}-07$ & $0,00 \%$ & & & & \\
\hline
\end{tabular}




\subsection{Acknowledgements}

I would particularly like to thank Jun.-Prof. Dr. Heinz Neumann for giving me the opportunity to work on this interesting project, the always open door for questions and discussions and his support over the last years.

Prof. Dr. Kai Tittmann I would like to thank for being the second reviewer of my PhD thesis and for participating in the thesis comittee meetings.

I would like to thank Dr. Cornelia Herrfurth and Dr. Tim Iven in the group of Prof. Dr. Ivo Feußner for the mass spectrometry measurements and their technical support. In this context I would also like to thank Sabine Freitag for the technical support on HPLC measurements.

All my colleagues in the department of applied synthetic biology I would like to thank for the good atmosphere in the group and many interesting conversations. It was fun working with you.

Particularly Lilli and Bryan I would like to thank for proofreading my thesis.

Sandra and Micha of the department of molecular structural biology I would like to thank amongst other things for many enjoyable lunch breaks and other fun "lab activities".

I would also like to thank all other colleagues for interesting and diverting conversations.

Jan Kampf and Florian Lenk have contributed in the scope of lab rotations to this thesis.

All my friends I would like to thank that they were at my side in the last years. Special thanks I would like to give to Tanja who did not only proofread my thesis but was always 
ready to lend an open ear, give encouraging words and give full support in all kinds of things. Special thanks also go to Katha for her support and untiring readiness for also fast digest of "Lesefutter" on short notice.

At last I would like to thank my family to whom this work is dedicated to for their versatile support during my studies and the time as PhD student. Thank you! 


\subsection{Curriculum vitae}

Svenja Heitmüller

Breite Straße 4

37077 Göttingen

E-Mail: Svenja.Heitmueller@gmx.de

Date of birth: 05.12.1981

Place of birth: Hameln

Nationality: german

Education and academic qualification:

since $03 / 2010$

$2002-2009$

$05 / 2001$

$1994-2001$

$1998-1999$

$1988-1994$
PhD programm " basic biology program" at Georg-August

University School of Science (GAUSS)

academic studies of chemistry at Georg-August University

Göttingen, graduation as “Diplom-Chemikerin”

allgemeine Hochschulreife (Abitur)

Friedrich-Schiller-Gymnasium, Hameln

Helena High School, Montana, USA

Grundschule Kirchohsen und Orientierungsstufe Emmerthal 
